\title{
Calcium at fertilization and in early development
}

\author{
Michael Whitaker
}

\begin{abstract}
Fertilization calcium waves are introduced and the evidence from which we can infer general mechanisms of these waves is presented. The two main classes of hypothesis put forward to explain the generation of the fertilization calcium wave are set out and it is concluded that initiation of the fertilization calcium wave can be most generally explained in inverterbrates by a mechanism in which an activating substance enters the egg from the sperm on sperm-egg fusion, activating the egg by stimulating phospholipase $\mathrm{C}$ activation through a src family kinase pathway and in mammals by the diffusion of a sperm-specific phospholipase $\mathrm{C}$ from sperm to egg on sperm-egg fusion. The fertilization calcium wave is then set into the context of cell cycle control and the mechanism of repetitive calcium spiking in mammalian eggs is investigated. Evidence that calcium signals control cell division in early embryos is reviewed, and it is concluded that calcium signals are essential at all three stages of cell division in early embryos. Evidence that phosphoinositide signalling pathways control the resumption of meiosis during oocyte maturation is considered. It is concluded on balance that the evidence points to a need for phosphoinositide/ calcium signalling during resumption of meiosis. Changes to the calcium signalling machinery occur during meiosis to enable the production of a calcium wave in the mature oocyte when it is fertilized; evidence that the shape and structure of the endoplasmic reticulum alters dynamically during maturation and after fertilization is reviewed and the link between ER dynamics and the cytoskeleton is discussed. There is evidence that calcium signalling plays a key part in the development of patterning in early embryos. Morphogenesis in ascidian, frog and zebrafish embryos is briefly described to provide the developmental context in which calcium signals act. Intracellular calcium waves that may play a role in axis formation in ascidian are discussed. Evidence that the Wingless/calcium signalling pathway is a strong ventralizing signal in Xenopus, mediated by phoshoinositide signalling is adumbrated. The central role that calcium channels play in morphogenetic movements during gastrulation and in ectodermal and mesodermal gene expression during late gastrulation is demonstrated. Experiments in zebrafish provide a strong indication that calcium signals are essential for pattern formation and organogenesis.
\end{abstract}

\section{INTRODUCTION}

As is well known, changes in intracellular free calcium concentration $\left(\left[\mathrm{Ca}_{\mathrm{i}}\right]\right)$ serve as signals. Physiologists can point to the ready examples of muscle contraction and synaptic transmission when explaining how a calcium signal works and what it does. If we include the heart in the list, then the three examples contain all the elements that make up calcium signalling systems: transmembrane calcium fluxes modulated by receptor and voltage-gated channels, pumps and antiporters; internal stores of calcium in the endoplasmic reticulum, mitochondria and other membrane bound organelles; calcium sensing proteins that interpret the calcium fluctuations and elicit various cellular responses. The three examples also suggest a tempting generalization, that calcium signals are involved in the control of rapid

Contact details: Institute of Cell \& Molecular Biosciences Faculty of Medical Sciences University of Newcastle Newcastle upon Tyne NE2 4HH UK Tel: +44 1912225264 Fax: +44 1912225164 Michael.Whitaker@ncl.ac.uk. 
and frequently repeated responses such as the muscle twitch, the pulse of neurotransmitter release and the heartbeat.

But as it turns out, most cell types contain a very similar portfolio of calcium signalling elements. Calcium signals are apparently ubiquitous $(41,96)$. They are present in somatic cells, and also in the germline in both sperm(110) and eggs(522). This review will take as its theme the calcium signals that occur in oocytes, eggs and embryos. Progress from oocyte to early embryo takes the form of a linear, irreversible programme of events. Each set of events must occur in strict sequence at an appropriate time. There is no repetition. The context of each calcium signal is different. As will become apparent, calcium signals in oocytes and embryos mark changes in cell state and are the milestones of the transitions that form the developmental programme.

Our understanding of the functional significance of these calcium signals ranges from very good to indifferent. A large, readily measured calcium increase like the fertilization calcium signal $(509,540,603,608)$ is now secure in its functional importance, thanks to the large body of data now is gathered. At the other extreme lie the small, sporadic calcium transients that occur as the early embryo moves towards and through gastrulation $(596,597)$ : we can guess that they may be important in pattern formation, but data are scanty. In the middle of this continuum lie the calcium signals that have been correlated with cell cycle events in oocytes and early embryos. We will start with the fertilization calcium signal, then discuss cell cycle-related calcium signals and finish with the sporadic calcium signals that mark later development.

It is also useful at the outset to consider the ways in which questions about cell regulation can be answered in these large germline cells. Genetic approaches are, on the whole, very difficult because only mutations that spare the mother but compromise the oocyte or early embryo are useful as analytic tools in oocytes, eggs and early embryos; widespread transcription of the zygote genome starts at about the time that the scope of this review begins to end. Such mutations, it appears, are rare. An example is a deletion of the mos gene. Mos protein is responsible for the mitotic arrest before fertilization in mammalian oocytes, its only role in development and beyond (580). Mos -/- mice have proved very useful, but mos is unusual in making such a crucial but brief appearance. Size, on the other hand, offers advantages. Oocytes are very large. Most work relies on microinjection methods and imaging, techniques that are more conveniently done in large cells. Calcium signals are now mostly measured using fluorescent calcium dyes. The development of laser scanning confocal microscopy has had a very big impact on the field. Dissecting the signalling pathways has been made much easier as recombinant construct that perturb elements of each pathway have been made. Green fluorescent protein-tagged Constructs have also been used very effectively. It will be possible eventually to understand the role of calcium signals in development without making extensive direct use of genetics, though of course genome sequence information will be essential in conceiving and designing molecular probes.

\section{A. Context}

The view that calcium might be essential for egg activation stretches back to Lillie and Heilbrunn $(204,327)$. It took its present form as a result of two crucial observations. The luminescent calcium sensor aequorin was used to demonstrate a striking calcium wave in a fish egg(170); a calcium increase was also recorded in sea urchin eggs at fertilization(509) and the calcium ionophore A23187 was shown to activate both vertebrate and invertebrate eggs and oocytes $(72,512,513)$. These two sets of experiments established that in most eggs the fertilizing sperm triggered a propagating calcium wave (228) that Jaffe has called a 
calcium explosion (233) and that this increase in intracellular calcium was sufficient to cause all the concomitants of egg activation (608).

The calcium responsible for egg activation was shown to be released from stores within the egg $(69,104,512)$. As it emerged that phosphoinositide lipids might be involved in a signalling pathway that led to release of calcium from internal stores $(35,37)$, it was found that there was a marked increase in turnover of the phosphoinositides at fertilization of sea urchin eggs(572). Berridge and his colleagues demonstrated that the product of PPI hydrolysis, $\mathrm{InsP}_{3}$, cause calcium release from permeabilized pancreatic cells(520); this led to the demonstration that microinjection of $\mathrm{InsP}_{3}$ into sea urchin eggs caused their activation (605). A model of fertilization emerged in which the fertilizing sperm triggered production of $\mathrm{InsP}_{3}$ that then generated a propagating calcium wave (reviewed in (603). The debate at that time turned around whether the sperm activated a receptor cascade within the egg, or whether an activating agent was introduced into the egg cytoplasm when sperm and egg fused. This debate continues more than ten years later and has been most recently reviewed in the context of the role of src-like tyrosine kinases in generating $\mathrm{InsP}_{3}$ at fertilization (231). The basic model remains intact. Here I intend to review in outline the various activation hypotheses and taking a different perspective, link what is known about egg calcium channels to the various proposed mechanisms for calcium release at fertilization and considering some of the possible targets of fertilization calcium signals.

There has also been an increasing amount of data gathered about fertilization calcium signals in eggs and oocytes of taxa other than echinoderms and mammals and of calcium signalling early in oocyte maturation. The comparative biology of calcium signaling in eggs and oocytes have been comprehensively summarised and critically reviewed (522). I will therefore not discuss these data exhaustively, instead making only crucial comparisons where they can help build a general framework for understanding the causes and consequences of the calcium signals from a perspective of cell cycle progression and control in oocytes as they pass through meiosis and into mitosis. Key model organisms have made a major contribution to our understanding of calcium and the causal mechanisms of development: frog, zebrafish, ascidian, sea urchin and starfish.

The third element of this article, the calcium signals that occur during early development, has not been comprehensively reviewed, though there are useful reviews and hypotheses in articles on calcium signals in development(36), in frog dorso-ventral axis formation (388) and in frog and zebrafish pattern formation(595-597).

\section{FERTILIZATION}

\section{Summary}

Fertilization calcium waves are introduced and the evidence from which we can infer general mechanisms of these waves is presented. The two main classes of hypothesis put forward to explain the generation of the fertilization calcium wave are set out and it is concluded that initiation of the fertilization calcium wave can be most generally explained in invertebrates by a mechanism in which an activating substance enters the egg from the sperm on sperm-egg fusion, activating the egg by stimulating phospholipase $\mathrm{C}$ activation through a src family kinase pathway and in mammals by the diffusion of a sperm-specific phospholipase $\mathrm{C}$ from sperm to egg on sperm-egg fusion. The fertilization calcium wave is then set into the context of cell cycle control and the mechanism of repetitive calcium spiking in mammalian eggs is investigated. 


\section{A. The basic model - calcium waves}

Sperm are small and eggs, large. Though calcium does not increase as a wave in all species (516), adaptation to this disparity of size has in most deuterostome species produced the fertilization calcium wave. Calcium first increases at the point of sperm-egg interaction and crosses the egg to the antipode (Figure 1). In mammalian oocytes and echinoderm eggs which are around $0.1 \mathrm{~mm}$ in diameter, the wave crosses the egg in about $2 \mathrm{~s}$ in mammals and about 20s in echinoderms $(116,196,234,246,540,603)$ and $C$. elegans (456); in $1 \mathrm{~mm}$ diameter frog eggs it takes several minutes $(55,157,164,602)$. Calcium wave velocities are constant at 5-50 $\mu \mathrm{m} \cdot \mathrm{s}^{-1}(238)$. Fertilization calcium waves are a subset of the class of calcium waves of this velocity that are found widely in both germline and somatic cells (234). Jaffe presents the evidence that these are, in terms of chemistry, reaction-diffusion waves analogous to the well known Belusov-Zhabotinsky reaction $(237,503)$.

The calcium wave triggered by the sperm can propagate even when the egg or oocyte is bathed in calcium-free media, indicating that it is due to release from internal stores $(68,69$, 104, 477). A major source of the participating calcium is the endoplasmic reticulum (163, $269,552)$ where calcium accumulation is driven by a $\operatorname{SERCA} \operatorname{pump}(243,272,300,309)$, as it is in many other cell types. ER-mediated calcium waves comprise the summation of elementary release events from either InsP $\mathrm{P}_{3}$ - or ryanodine-receptors $(77,256,331,347)$. Calcium released from a receptor or cluster of receptors can diffuse to neighbouring receptors, triggering further calcium release (calcium-induced calcium release: CICR; (137, $256,501)$ ). This mechanism procedes by reaction (calcium triggered activation of the release channel) and diffusion (of calcium to neighbouring receptors) and is thus a good candidate for the mechanism underlying fertilization calcium waves. Evidence in its favour: that microinjection of calcium can trigger a propagating wave $(198,383)$; that ruthenium red, an RyR antagonist, reduces the propagation velocity of the wave in sea urchin eggs $(164,374)$; that a calcium response can be triggered in sea urchin eggs by calcium influx after sensitizing the $\mathrm{RyR}(355)$; and that an inhibitory antibody directed against the $\mathrm{InsP}_{3}$ receptor channel blocks both $\mathrm{InsP}_{3}$ - and calcium-induced calcium release.(377, 378)

In general, however, the fertilization calcium wave is carried by the $\operatorname{InsP}_{3} \mathrm{R}(522)$; only in fish and echinoderm eggs does there appear to be a substantial contribution from the RyR $(154,164,377,435,458)$. While the Ins $\mathrm{P}_{3} \mathrm{R}$ is sensitive to calcium and can undergo CICR, production and diffusion of $\mathrm{InsP}_{3}$ can also participate in the reaction diffusion mechanism underlying $\operatorname{InsP}_{3} \mathrm{R}$-mediated calcium waves $(16,255)$. There is evidence of calciumdependent InsP $\mathrm{P}_{3}$ production during the fertilization calcium wave $(88,94,517,606)$. Support for the idea from inhibition experiments is mixed. The $\mathrm{InsP}_{3}$ antagonist heparin delays the onset of the wave, without appearing to alter its velocity (105), while a dominant negative $\mathrm{PH}$ domain inhibitor of PLC $\gamma$ not only delays the onset, but also appears to diminish its propagation (482). I'll discuss this disparity further when we come to consider how the wave is initiated.

It is not merely the participation of the echinoderm RyR (491) in the propagation of the fertilization calcium wave that marks out the egg as different from frog and mammals, though similar to medaka and sea bream oocytes $(154,435)$. Study of the calcium release mechanisms of unfertilized eggs has uncovered two novel calcium-releasing messengers $(13,82,121,161,165,408,464,611)$, in part perhaps because of the availability of readilyprepared egg homogenates that both sequester and release calcium in a reproducible and apparently physiological way (163). The first to be identified was cyclic ADPribose (cADPr), the product of a ADP-ribosyl cyclase acting on $\beta_{N A D}+(307,313)$. Cyclic $\mathrm{ADPr}$ releases calcium quite independently of $\mathrm{Ins}_{3}$, its pharmacology indicating that it stimulates release via the RyR (161). The receptor for cADPr has not yet been identified; the only additional information we have is that its action requires calmodulin, which appears to 
increase affinity for cADPr and to increase the sensitivity of the RyR to CICR by several orders of magnitude $(310,311,558)$. Cyclic ADPr has been shown to mediate calcium signalling in pancreatic acinar and $\beta$-cells and in heart $(159,223,307,560)$. Antagonists of cADPr do not alter the propagation of the fertilization calcium wave in sea urchin eggs(316), but appear to curtail the long falling tail of the transient(301), indicating that cADPr acts late during the fertilization calcium response. In eggs of species in which RyR do not make a large contribution to the initial fertilization calcium wave, cADPr does not cause global calcium release when microinjected $(22,276)$, despite the presence of the ryanodine receptor $(22,534)$. In these mammalian eggs, calcium release from RyR at the egg periphery may nonetheless contribute to cortical granule exocyosis (276) and to sustaining the multiple calcium oscillations that follow fertilization (534). In ascidian oocytes, cADPr opens cation channels $(13,611)$.

The same enzyme that generates cADPr from $\beta \mathrm{NAD}^{+}$working in a different (base exchange) mode can generate nicotinic acid adenine dinucleotide phosphate (NAADP) from NADP and nicotinic acid in vitro $(3,308)$. NAADP is a very interesting calcium releasing messenger on two counts. First, its desensitization and activation curves in sea urchin eggs barely overlap: nanomolar concentrations of NAADP can desensitize the receptor to the tens of $\mathrm{nM}$ concentrations that usually produces rapid and complete calcium release; its binding receptor in sea urchin eggs does not appear to be the the RyR or $\operatorname{InsP}_{3} \mathrm{R}$ (34), though it has also been shown that physiological concentrations of NAADP can activate purified skeletal muscle RyR(214) and that NAADP can release calcium from the nuclear envelope of pancreatic acinar cells(168). Second, NAADP releases calcium from an internal store, but in sea urchin eggs, this is not the ER. There is every indication in sea urchin eggs that its target is a channel on a lysosomal class membrane compartment (87). This calcium store is filled by a $\mathrm{Ca} / \mathrm{H}$ antiporter, driven by the compartment's low $\mathrm{pH}$. Also curious is the observation that the store retains calcium for long periods even once the antiporter in inhibited, in some contrast to the rapid depletion of ER calcium when the ER SERCA pump is inhibited (87). Lee has shown that uncaging NAADP in sea urchin eggs leads to calcium oscillations (2) and has suggested that calcium oscillations may reflect transfer between $\mathrm{InsP}_{3}$ and NAADP-sensitive stores (306). Galione puts forward the similar idea that NAADP stores may be used by cells to enhance and re-inforce InsP $_{3}$ - and RyR-mediated calcium signals. NAADP-induced calcium release is densensitized after fertilization in sea urchin (425) and prior uncaging of caged NAADP reduces the rate of rise of a subsequent fertilization calcium transient (165). However, NAADP-induced calcium release does not itself induce a regenerative, propagating calcium wave in sea urchin eggs $(85,165)$, nor does inactivating the NAADP pathway prevent inititation of the fertilization calcium transient and propagation of the calcium wave (86). Similarly in starfish oocytes at fertilization, photorelease of NAADP results in a cortical calcium increase that sometimes fails to spread (408) and sometimes propagates as a high velocity wave at $150 \mu \mathrm{m} \cdot \mathrm{s}^{-1}$, an order of magnitude faster than a fertilization calcium wave $(329,381)$. In sea urchin, the rapid, transient cortical calcium increase at fertilization is known to be due to activation of voltage activated L-type calcium channels $(70,111)$. This cortical flash is absent when the NAADP pathway is desensitized(86). Similarly in starfish, desensitization of the NAADP pathway leads to loss of the activation current carried by calcium (380). These observations suggest that NAADP may play an important role in regulating membrane currents at fertilization.

The idea is borne out by experiments that show that in mature starfish oocytes the NAADPinduced calcium increase requires extracellular calcium and is blocked by the calcium channel blockers nifedipine, verapamil and SKF96365 $(329,464)$. cADPr also triggers local cortical calcium increases that are blocked by nifidipine and require extracellular calcium and which contribute to cortical granule exocytosis $(408,464)$. cADPr has also been reported to activate the channels that activate starfish fertilization cation currents 
independently of calcium(611). In ascidian oocytes at fertilization, NAADP signaling downregulates a plasma membrane calcium channel, cADPr signaling causes cortical granule exocytosis, while Ins $\mathrm{P}_{3}$ generates the fertilization calcium wave, with NAADP necessary for subsequent calcium oscillations in conjunction with $\operatorname{InsP}_{3}(13)$.

There are observations that suggest that NAADP may initiate the fertilization wave in starfish. Enucleated mature oocytes have a much reduced sensitivity to $\mathrm{InsP}_{3}$ while retaining sensitivity to NAADP(329). When fertilized, these oocytes show a much attenuated propagation of the calcium wave and do not undergo cortical granule exocytosis, but the calcium increase close to the point of sperm entry remains strong. In sea urchin eggs, however, initation and propagation of the calcium wave is unaffected by inactivation of NAADP signaling, though the duration of the transient is shortened and, interestingly, the rapid cortical calcium increase due to voltage-gated calcium influx is abolished (86). This last observation has an echo in the finding that NAADP-induced calcium release from internal calcium stores can be prevented by a dihydropyridine blocker of voltage-gated calcium channels (306).

The sea urchin egg finds itself in the vanguard of calcium signalling; study of its calcium signaling pathways has helped uncover three messengers: $\mathrm{InsP}_{3}, \mathrm{cADPr}$ and NAADP. Ironically, despite this cornucopia, only $\mathrm{InsP}_{3}$ appears to contribute to the initiation of the fertilization calcium wave in sea urchin, the other two messengers giving it a boost and longevity.

\section{B. How a sperm activates an egg - a survey of experimental approaches}

Fertilization calcium waves are autonomous and a property of eggs and oocytes: they can be triggered by microinjection of calcium or $\mathrm{InsP}_{3}$ or, indeed, by a needle prick. The answer to how a sperm activates an egg is a simple one in outline: interaction of the sperm with the egg triggers a signal transduction pathway that initiates the autonomous calcium wave in the egg. What about the molecular detail? After almost twenty years of research much of the detail is still missing. It also begins to look as though the detail will differ from one species to another, despite the strong conservation of the calcium wave mechanism itself. Before going on to the detail, it is worth asking why it has been so difficult to uncover.

The difficulty turns on the size disparities of egg and sperm. The area of interaction between egg and sperm can be as little as $100 \mathrm{~nm}$ in diameter which is about $0.025 \%$ of the egg surface area of a $100 \mu \mathrm{m}$ egg; similarly, if the initial signalling cascade begins in a $1 \mu \mathrm{m}^{3}$ volume just beneath the point of sperm egg interaction, this represents $0.0002 \%$ of the egg volume. Detecting the early biochemical changes during sperm-egg interaction is beyond the limits of grind and measure approaches: a messenger would have to increase 0.5 million fold in the $1 \mu \mathrm{m}^{3}$ volume to represent a doubling over background concentration when measured by analyzing whole eggs. Not surprisingly, physiological approaches, electrophysiology, fluorescence imaging and microinjection, in single eggs have yielded the best insights into the detail of the early stages of sperm-egg interaction.

1. Electrophysiology-Echinoderm eggs depolarize at fertilization (514) (Figure 2). The depolarization is a physiological response to the first interacting sperm that reduces the probability of interaction with sperm that engage subsequently $(229,230)$, the so called fast block to polyspermy. It is fast, compared to the other timescales at fertilization, the membrane potential reaching $20 \mathrm{mV}$ positive within $20 \mathrm{msec}(70,111,229,341)$. This rapid depolarization is due to activation of voltage gated L-type calcium channels $(70,111,607)$, the only further mention, incidentally, to voltage-gated ion channels that will be made in this review, save for the brief discussion of their role at fertilization in the ascidian and in starfish below, until we come to discuss neural induction in the embryo. The opening of 
these channels is triggered by a small monovalent cation current(71, 107, 342, 352, 537). Very elegant work by Chambers and his colleagues showed that the onset of this current is simultaneous with a capacitance increase in the small region of egg membrane with which the sperm is interacting, a capacitance increase due to the fusion of sperm and egg (334, 352). Sperm-egg fusion is reversible in some circumstances (385) and current and capacitance always went hand in hand. The model that emerges from these experiments is of sperm egg fusion providing a conduction pathway from egg to sperm that allows inward current through existing channels in the sperm membrane to depolarize the egg membrane, triggering a calcium action potential and thus the fast polyspermy block. Thus once a single sperm has fused, it is difficult for a second sperm to do so (why is unclear), suggesting that sperm egg fusion is fertilization's Rubicon in the sea urchin. This mechanism neatly and within the space of $20 \mathrm{~ms}$ ensures fertilization but prevents polyspermy which is fatal developmentally in the sea urchin.

In the sea urchin, no increase in cytoplasmic calcium is needed for sperm-egg fusion (537) and calcium can also play an inhibitory role in the incorporation of the sperm into the egg after fusion. Once cytoplasmic continuity is established, the egg extends its plasma membrane to engulf the sperm in its fertilization cone. Movement of the sperm further into the cytoplasm requires actin filaments(199) and is facilitated by depolarization(354). Chambers and his team found that when eggs were voltage clamped at negative potentials close to the resting potential of the unfertilized egg, incorporation of the sperm into the egg failed; sperm-egg fusion had occurred but was often reversed, the egg nonetheless activating (353). By varying the calcium concentration of sea water, by measuring the membrane voltage inhibition function and by using calcium channel blockers, it was possible to demonstrate that the inhibition of sperm incorporation was due to the larger calcium influx at more negative potentials due to the increased electrochemical potential across the membrane relative to controls; note that this calcium flux was almost certainly through the sperm plasma membrane and into the egg via the sperm-egg fusion pore, as the egg's L-type calcium channels were not activated under these voltage clamp conditions. The depolarization at fertilization thus serves a second function at fertilization beyond the block to polyspermy, that of ensuring sperm incorporation. These experiments can be compared with observations on sperm incorporation made with two inhibitors of the fertilization wave, EGTA and neomycin (537). The calcium chelator EGTA completely blocks the fertilization calcium wave, but sperm incorporation occurs normally; indeed eggs are polyspermic, because the fast polyspermy block is not absolute, reducing fertilization probability by a factor of around 20(24). The absolute block to polyspermy is provided by the calciumdependent exocytosis of cortical granules that cause elevation of the fertilization envelope, a mechanical barrier to polyspermy. In contrast, neomycin, a blocker of polyphosphoinositide (PPI) metabolism, while abolishing the fertilization calcium wave as might be expected, also causes reversible sperm-egg fusion and prevents sperm incorporation (537), raising the possibility that PPI metabolism is required for sperm egg incorporation. The production of polyphosphoinositide lipids may be necessary for the membrane anchoring of actin filaments $(224,287)$.

Electrophysiology has uncovered two important roles for the calcium channel-dependent depolarization at fertilization: the polyspermy block and the facilitation of sperm incorporation. Equally important, it has provided key information about the timing of sperm egg fusion relative to calcium influx. We shall return to this later.

Eggs and oocytes of other phyla have less informative electrophysiology. The depolarization polyspermy block is found in Rana, Xenopus and ascidian $(75,183,296,593)$, but there is no information from electrophysiology about sperm-egg fusion in these species. Mouse oocytes show insignificant $(<5 \mathrm{mV})$ membrane potential variation at fertilization and there is no 
evidence of an electrical block to polyspermy $(222,232)$, though the block due to calciumdependent cortical granule exocytosis operates(351). Hamster oocytes have very marked episodic hyperpolarizations(376) after fertilization due to calcium-dependent potassium channel activation by the calcium oscillations we shall later discuss; ascidian oocytes similarly show episodic depolarisations(184); their physiological function, if any, is unclear.

2. Fluorescence imaging-One can visualize sperm-egg fusion using confocal fluorescence imaging. A fluorescent dye microinjected into an egg will diffuse into the sperm when in fuses (Figure 3). A fortunate confocal section will reveal the fertilizing sperm. If a calcium-sensitive fluorescent dye is used, the relative timings of sperm-egg fusion and the initiation of the fertilization calcium wave can be determined. In sea urchin eggs, dye transfer precedes the initiation of the calcium signal by $15-20$ s $(360,538)$; in mouse dye transfer into the sperm head and tail antecedes the generation of the first fertilization calcium wave by a minute or more $(246,299,538)$ and transfer of a $200 \mathrm{kD}$ protein also takes place long before the calcium wave is initiated(246).

The first fertilization calcium wave to be discovered was visualized not by fluorescence, but by luminescence(170). The calcium-stimulated photoprotein aequorin was microinjected into medaka (Oryzias latipes) eggs. These and other fish eggs are large and have a thin rim of cytoplasm just beneath the plasma membrane, surrounding the central yolk, ideal properties for imaging aequorin luminescence. Calcium-stimulated light emission ran around the cytoplasmic layer, meeting again at the opposite pole. Later, aequorin was used to detect a fertilization calcium wave in sea urchin $(133,135,540)$, starfish(134), ascidian $(53,502-504,635)$ and zebrafish eggs . However, while aequorin, as we shall see later, is well suited to measuring calcium gradients, it is severely photon-limited and lacks the spatial resolution available with confocal fluorescence methods.

Confocal fluorescence imaging has defined the characteristics of fertilization calcium waves; they originate at the point of sperm entry and cross the egg with a spherical wavefront whose geometry is modified by the boundary curvature of the egg as they propagate $(157,330,359,397,408,485,524,526,527)$. Confocal imaging has also revealed the substructure of the repetitive fertilization calcium responses $(375,502,505)$ of ascidians and mammalian eggs . In ascidians, the wave originator moves from the point of sperm entry laterally towards the vegetal pole with each successive wave $(130,358)$. In hamster oocytes, the first few calcium transients take the form of waves originating at the point of sperm entry; subsequent transients were reported to rise uniformly throughout the cytoplasm(375); later it was found in mouse that the subsequent transients tended to arise in the cortex of the vegetal pole of the $\operatorname{egg}(116)$, as in the ascidian, where clusters of endoplasmic reticulum are denser (274). The same pattern is seen in a nemertean worm(524). We shall return to consider the significance of these patterns later.

In sea urchin eggs, calcium indicator dyes detect the calcium influx that occurs when the egg depolarizes $(355,485)$ (Figure 2). This, as we have seen, is coincident with sperm-egg fusion. Though in starfish oocytes, calcium influx and wave initiation occur within a few seconds of one another and each may precede the other (380), in sea urchin eggs, a remarkably long time elapses between sperm-egg fusion as defined by calcium influx and the initiation of the fertilization calcium wave: abount 15s in Lytechinus pictus, equivalent to the time it then takes for the fertilization wave to cross the $\operatorname{egg}(359,485,527,603)$. This latent period, first defined from kinetic analyses of fertilization rate defined by fertilization envelope elevation(17) is a characteristic feature of fertilization. The time from sperm egg fusion to the initiation of the first fertilization wave in mouse is a minute or more. Response latencies of this degree imply a high amount of cooperativity in the signalling response. At fertilization, cooperativity is inherent in the CICR component of the fertilization calcium 
wave (256) which goes some way to help our understanding. However, even now there are scant data on changes in second messengers during the latent period: it remains dark as ever (603).

One approach to measuring phosphoinositides and $\mathrm{InsP}_{3}$ has been to use appropriate fluorescent PH domains from, for example, PLC $\delta$ (Figure 4). The PH domain shows affinity for both PtdInsP $\mathrm{P}_{2}$ and $\mathrm{InsP}_{3}$ (318) and, coupled to GFP, has been identified as an $\mathrm{InsP}_{3}$ indicator, moving from plasma membrane to cytoplasm as $\mathrm{InsP}_{3}$ increases $(213,576)$. In mouse eggs the PH-GFP indicator shows a slow and steady increase in localization to the plasma membrane throughout the period after fertilization during which repetitive calcium pulses occur (197). Release of exogenous $\mathrm{InsP}_{3}$ into the cytoplasm by photorelease of caged $\mathrm{InsP}_{3}$ was able to strip the indicator from the plasma membrane only at doses far higher than required to cause the calcium oscillations themselves. The indicator thus has a far higher apparent affinity for plasma membrane PtdIns $\mathrm{P}_{2}$ than for cytoplasmic $\mathrm{InsP}_{3}$ at its active concentration. The absence of periodic changes in plasma membrane $\mathrm{PtdInsP}_{2}$ as revealed by the $\mathrm{PH}$ domain indicator implies that the calcium pulses are not accompanied by episodes of PtdIns $\mathrm{P}_{2}$ hydrolysis, favouring a mechanism in which periodic calcium oscillations are generated by constant, though enhanced, concentrations of $\operatorname{InsP}_{3}(150,245,255,386,473)$. The sustained increase in plasma membrane PH domain-GFP fluorescence could be abrogated by blocking cortical granule exocytosis using a toxin directed against the fusion machinery. The significance of this latter observation is unclear. One possibility is that the addition of the granule membrane leads to lateral diffusion of $\mathrm{PtdInsP}_{2}$ and dequenching of the GFP fluorescence. Our unpublished observations using an identical PH-GFP in sea urchin eggs at fertilization show local increases in fluorescence coincident with cortical granule fusion events, supporting this interpretation. Similarly, a steady increase of PH-GFP is seen in ascidian oocytes after fertilization. There is an accumulation of fluorescence at the contraction pole, but no evidence of oscillatory behaviour (65). One disadvantage of the PHGFP probe is that its temporal resolution is limited by its slow diffusion relative to the much smaller InsP $\mathrm{P}_{3}$ molecule. The slow rise of cytoplasmic PH-GFP fluorescence after fertilization in the sea urchin egg has led to the conclusion that the $\mathrm{InsP}_{3}$ increase may be very slow, indeed much slower that the onset of the fertilization transient (557). However, our own unpublished diffusion modeling suggests that the indicator cannot track a rapid increase in $\mathrm{InsP}_{3}$.

The recent availability of fluorescent indicators of nitric oxide prompted a study in sea urchin eggs, as it was known that NO could induce a calcium transient in unfertilized eggs $(292,617)$. Using the indicator DAF-2 two increases in fluorescence were observed: an early transient increase soon after insemination, and a later sustained increase(292). This was an indication that NO might increase during the latent period and so be a candidate for the messenger that initiates the calcium wave. Both sperm and egg contain nNOS, a calcium activated nitric oxide synthase (292). A subsequent report found no indication of such an early increase, however. Using an analogous NO indicator (DAF_FM) less sensitive to the $\mathrm{pH}$ increase $(241,487)$ that accompanies fertilization in sea urchin eggs $(608)$, only the later NO increase was detected (301). No alterations in NO were detected in either mouse or ascidian oocytes at fertilization nor was fertilization prevented by an NO inhibitor Lnitrosomethylarginine; a calcium-dependent NOS activity was however detected and the same inhibitor could block NO production by this route(221)

3. Microinjection-Analysis of the effects of agonists and inhibitors is most readily achieved in eggs and oocytes by microinjection. A list of agonists that activate eggs includes: $\operatorname{InsP}_{3}(47,358,396,430,447,464,536,540,571,605)$, cADPr $(97,159,312,408$, 464), NAADP $(13,81,97,380,381,408,425,464,559)$, GTP $\gamma \mathrm{S}(105,273,374,571)$, botulinum C3 toxin (a rho GTPase activator)(565), src kinase (176, 563), cGMP (601), nitric 
oxide (189, 221, 617), latrunculin A (330) and an extract of sperm from various species (42, $108,120,218,295,325,357,412,418,523,529,533,577,609,627)$. This class of experiment illustrates that eggs and oocytes possess signalling pathways that can be stimulated by these agonists to generate a calcium signal. That such a pathway exists is not, of course, evidence that it operates at fertilization. As an illustration, we can take GTP $\gamma \mathrm{S}$, a G-protein agonist. Though GTP- $\gamma$-S activates sea urchin eggs(571), the G-protein antagonist GDP $\beta S$ does not prevent the initiation of the fertilization calcium transient by sperm (105) (it does however, prevent cortical granule exocytosis]. Echinoderm eggs clearly possess a trimeric G-protein/PLC signalling pathway; indeed, if an exogenous G-protein linked receptor is expressed in starfish eggs by microinjection of the appropriate mRNA, then the oocytes, once mature, can be activated by exogenous 5-hydroxytryptamine $(52,489)$. Mouse oocytes can be activated in a similar way by expression of the muscarinic acetylcholine receptor (615). Nonetheless, this signalling pathway does not operate at fertilization (453, 614). Eggs and oocytes are promiscuous in their responses to agonists, perhaps because several latent signalling pathways are present in the unfertilized egg, not for use at fertilization, but for use at later stages of early development. It should be remembered that early development of embryos takes place largely in the context of stored maternal proteins and mRNA; significant transcription of zygotic genes takes place only at the mid-blastula stage $(112,194,195)$.

A list of antagonists that block or delay the initiation of the fertilization calcium transient includes: BAPTA $(192,270,271,359,516,537,611)$, EGTA $(376,540,568,639)$, oxyhemoglobin (an NO scavenger; yes:(292); no:(301), heparin $(105,164,276,312,358$, 384, 407, 458, 525, 559), an InsP $\mathrm{P}_{3}$ chelator(227), U73122 (a PLC antagonist: (117, 131, $317)$, dominant negative domains of PLC $\gamma(60,61,452,453,482)$ [but not mammalian oocytes:(363)] and of the src kinase family $(4,174,264,452)$, antibodies to a src kinase (also an agonist: (175)), genistein and tyrphostin (tyrosine kinase antagonists (18, 131, 178, $486)$ and neomycin $(244,537,540,568)$. Antagonists that fail to block the fertilization calcium signal include (in addition to GDP $\beta S$ ): 8-Br- and 8-amino-cADPr $(13,312,316$, 458), RcAMP-S (a cGMP antagonist (316)), ryanodine (164, 276, 359, 407, 485, 524).

This broad survey (Table 1) suggests a pattern in which in invertebrates (and possibly frog) a src family tyrosine kinase pathway $(89,469,563)$ may activate PLC $\gamma$ at fertilization to initiate the fertilization calcium wave (452). The exception is the mouse oocyte (363) and likely mammalian oocytes in general.

4. Biochemical analysis-I have already pointed out the difficulties inherent in biochemical measurements of the potentially very localized production of signalling molecules at the site of sperm-egg interaction. Nonetheless, it is logical to measure during fertilization the concentrations or turnover of signalling molecules known to activate eggs and oocytes. In fact most of these measurements have been made in sea urchin eggs, some in frog eggs, because mammalian oocytes are very hard to obtain in sufficient quantity for biochemistry.

i. Phosphoinositides: Phosphoinositide signalling involves a cycle of synthesis and hydrolysis(94). The major signalling substrate phosphatidylinositol bisphosphate $\mathrm{PtdInsP}_{2}$ is made by successive phosphorylation of phosphatidylinositol lipid. Hydrolysis of $\mathrm{PtdInsP}_{2}$ by a phospholipase $\mathrm{C}$ generates $\mathrm{Ins}_{3}$. $\mathrm{InsP}_{3}$ is degraded by phosphatases, generating inositol that is then used to resynthesize phosphatidyl inositol. The components of the cycle can be radioactively labeled using $\left[{ }^{3} \mathrm{H}\right]$-inositol or $\left[{ }^{32} \mathrm{P}\right]$-ATP. Turnover through the cycle increases markedly during fertilization and early development in the sea urchin $(88,94,449$, 572). Within 20s of fertilization, turnover has increased a thousand fold(88), with a net 
doubling of labeled PtdInsP $2(88,572)$ and a concomitant halving of its precursor, phosphatidylinositol (251).

ii. Ins $\mathbf{P}_{\mathbf{3}}:\left[{ }^{3} \mathrm{H}\right]-\mathrm{InsP}_{3}$ rises within 20 s of insemination and then falls at 60 s before rising again to a new plateau (94). This temporal pattern coincides with the timing of the fertilization calcium transient in the egg population though it appears that the initial increase in $\left[{ }^{3} \mathrm{H}\right]-\mathrm{InsP}_{3}$ precedes the calcium transient peak in the egg population (88). A proportion of the response may be due to calcium stimulation of a PLC, since labeled sea urchin egg plasma membrane generates $\left[{ }^{3} \mathrm{H}\right]-\mathrm{InsP}_{3}$ when physiological micromolar concentrations of calcium are added (604). Labelling in these experiments did not reach equilibrium, so it is difficult to convert these data into estimates of concentration.

Competition assays using exogenous radiolabel are a better way to estimate concentration changes. In sea urchin, $\mathrm{InsP}_{3}$ concentrations rise to $0.2-0.3 \mu \mathrm{M}$ in 20-30s (293) further increasing to $\sim 1 \mu \mathrm{M}$ at 120 s $(293,317)$, while in Xenopus, concentrations rise much more slowly to $0.5 \mu \mathrm{M}(517,518)$, declining over 10 minutes, as befits the much longer calcium wave in this large egg. No measurements of $\mathrm{InsP}_{3}$ have been made in mammalian oocytes.

iii. cADPr: CyclicADPribose increases to a peak of 50-200nM at 30-60s after insemination of sea urchin eggs, falling back to resting levels again by 150 s after insemination $(293,301)$.

iv. NAADP: NAADP rises in sperm to supramicromolar levels when sperm interact with the egg jelly coat $(45,86)$ and it is estimated that locally, these levels are attained at the point of sperm-egg fusion, while diffusion through the cytoplasm will result in a concentration of 1 $\mu \mathrm{M}(86)$.

v. Cyclic GMP: Cyclic GMP rises rapidly to a peak of 20-100nM at around 25s after insemination of sea urchin eggs $(90,293)$

vi. Tyrosine phosphorylation: Fyn kinase activity is reported to increase within 2 min after insemination in sea urchin (262); tyrosine kinases activity identified by phosphotyrosine antibodies increase with a similar time course (91); there is also a slow, long-lasting increase in tyrosine kinase activity after fertilization (263).

It is very difficult to draw conclusions from any temporal precedence of messengers in the sea urchin egg, as they all increase at comparable times, slightly preceding the fertilization calcium transient. However, a comparison of the reported concentrations with the efficacy of each messenger when microinjected is interesting: for cGMP, measured concentrations are 20-100 nM, while around 5-10 $\mu \mathrm{M}$ are needed to activate eggs $(293,601)$; for cADPribose, the figures are $150 \mathrm{nM}$ and $1-200 \mathrm{nM}(160,312)$, for NAADP , $1 \mu \mathrm{M}$ and 50nM $(86,165)$ and for InsP $\mathrm{P}_{3}, 1-100 \mu \mathrm{M}$ and $2 \mathrm{nM}(605)$. cGMP seems to be the most unlikely candidate for the activating messenger at fertilization on this purely quantitative argument.

\section{How a sperm activates an egg - a survey of hypotheses}

The title gives the game away. We don't yet have a definitive answer to the question of how a sperm activates the fertilization calcium wave. Nil desperandum; we are close to the answer. There are two classes of hypothesis(603). The first class imagines that the sperm activates a signal transduction receptor, much as a hormone might; the second class is based around the idea that sperm-egg fusion is the event that initiates the fertilization calcium wave. 
1. Transduction via a sperm receptor-Eggs must capture sperm if they are to be fertilized. In species that are external fertilizers, they must capture the right sperm in order to prevent cross species hybrids. This cell-cell recognition process is a key element in evolutionary speciation $(542,575)$. Cell-cell recognition processes at fertilization are likely to be a subset of the cell-cell recognition mechanisms that sort cells in an organism(147). These mechanisms generalize into cell-matrix and cell-cell interactions $(12,109)$. In the context of fertilization, the matrix is the egg coat, proteoglycan polymers $(66,191,480,481$, $490,592)$. One important component of the species-specificity of fertilization is the response of the sperm to this egg coat. Homologous sperm interact with the egg coat and undergo the acrosome reaction $(6,46,592,625)$. The acrosome reaction is a specific response to the coat with its own signal transduction pathways(110) and involves the exocytosis of the acrosomal vesicle which contains proteins and enzymes that dissolve the egg coat, allowing the sperm to pass through it. In broad terms, this is analogous to matrix remodeling $(143,521)$. Once the sperm reaches the egg, a cell-cell recognition process occurs.

In mammals, the cell-cell recognition process is mediated by an integrin/disintegrin interaction involving ADAM proteases and the CD9 tetraspannin fusion protein $(83,143$, $144,146,248,369,638$ ). An ADAM-based activation mechanism has also been proposed in frog (406). In sea urchin, the acrosome reaction unmasks an adhesion protein bindin, a very hydrophobic protein with affinity for carbohydrate (177) whose receptor has recently been identified (250) (Glabe, personal communication). Cell-cell recognition is the second species-specific interaction at fertilization (592). One class of hypotheses of egg activation postulates that this sperm-egg receptor interaction includes a signal transduction component that activates the egg.

Certainly, integrin receptors can transduce a transmembrane signal to generate a calcium response in general (12). Peptides containing the RDG integrin recognition motif can induce activation of frog and bovine oocytes $(57,226,581)$ and inhibit sperm binding and fusion (57). It has also been reported that application of bindin to sea urchin eggs can induce activation; however, the bindin receptor has no obvious signal transduction motifs (Glabe, personal communication). An earlier report of an egg membrane receptor for sperm (5) that possessed signal transduction motifs has not been confirmed. Nonetheless, it remains a possibility in mammalian oocytes that integrin signalling may activate tyrosine kinase pathways (623), presumably distinct from the PLC $\gamma$ pathway that has been shown not to operate at fertilization (363).

2. Transduction as a consequence of sperm-egg fusion-The second class of hypotheses that attempt to account for egg activation take as a premiss that activation requires fusion of sperm and egg. Whatever the merit of these hypotheses per se, they beg the question of how sperm-egg fusion occurs. The only indication of a mechanism lies in the observation that the hydrophobic sea urchin egg acrosomal protein bindin can induce fusion of lipid vesicles (177) and that the CD9 fusion protein of mammalian oocytes is involved in, for example, myocyte fusion(248). The implication of this observation is that once sperm and egg plasma membrane are glued together with bindin or integrin, then fusion will occur. There are no comparable data in other species.

Though we are ignorant of the mechanism of sperm egg fusion, it doubtless occurs. The distinguishing feature of the second class of hypotheses is that activation occurs as a consequence of sperm egg fusion. Does the sperm act as a conduit or a vehicle?

The conduit hypothesis is exemplified by the idea that, once fusion has occurred, calcium enters the egg by way of calcium channels in the sperm membrane $(235,626)$. This local calcium entry then sets off the calcium wave through a CICR mechanism. The idea is 
attractive in its simplicity. CICR would then be responsible both for the initiation and propagation of the fertilization calcium wave. It is a pity that this straightforward idea faces certain difficulties. The most telling is that acrosome-reacted sperm can activate sea urchin eggs in sea water that lacks calcium $(68,69,139)$; it is hard to envisage how a calcium flux through the sperm could be sustained in sea water where calcium concentrations are lower or comparable to resting concentrations in the egg(246). Another argument against the conjecture is that in sea urchin eggs no local increase in calcium concentration in the region of sperm egg contact is apparent until the calcium wave initiates(538). It could reasonably be argued that the calcium required to initiate the fertilization calcium wave may not be readily detectable. However, as we have seen, the cortical calcium increase that results from activation of voltage-dependent calcium channels at fertilization is certainly detectable (485, 538). Moreover, it takes many such action potentials and the CICR sensitizer thimerosal (355) to initiate a calcium transient via CICR.

The idea that the sperm is the vehicle that transmits an activating messenger to the egg once sperm egg fusion occurs has been a recurrent theme in the field $(99,108,162,219,277,325$, $451,452,465,523,533,609,610,624,627)$, based on the observations that extracts of sperm cytoplasm will induce calcium transients when microinjected into eggs. Though the phenomenon can be demonstrated in echinoderm, ascidian and mammalian eggs, most of the effort to identify the factor involved has been directed towards mammalian eggs. The main reason for this is the characteristic signature of the repetitive fertilization calcium transients in mammalian eggs. As we have seen, the promiscuous response of echinoderm eggs to activating agents makes them less attractive a model for isolating a calcium-releasing factor.

Heat sensitivity and molecular mass indicated that the factor was a protein (533). Using the mammalian fertilization calcium signature as a bioassay to test fractions, the factor was originally identified as a hexose phosphate isomerase(421). That this enzyme activity could generate calcium signals would have provided a novel slant on calcium signalling pathways, but the identification turned out to be mistaken(622). The well characterized sea urchin egg homogenate was used to demonstrate that the factor possessed a phospholipase $\mathrm{C}$ activity $(242,244,443)$ and it is now reported that the factor is a testis-specific PLC of novel class designated PLC $\zeta(100,474)$. PLC $\zeta$ has been identified as tracking the active fraction from sperm, PLC $\zeta$ mRNA will induce the characteristic calcium signature when introduced into mammalian eggs and PLC $\zeta$ antibodies can be used to immunodeplete the calcium releasing activity from extracts. Recombinant PLC $\zeta$ protein also produces the characteristic calcium signature when microinjected into eggs (280). It is calculated that a single sperm (474) contains sufficient of the enzyme to activate an egg. Inhibition of fertilization by inhibition or knockout of PLC $\zeta$ remains to be demonstrated. It is interesting to note that this phospholipase activity appears much more potent than PLC $\gamma(364)$, which, as we have said, does not appear to play a major role in mammalian fertilization(363).

Ascidian oocytes resemble those of mammals in having a characteristic calcium signature at fertilization. A start has been made on isolating and characterizing an ascidian sperm factor $(295,357,609)$ (Keith Jones, Alex McDougall and Tony O'Sullivan, personal communication). The ascidian sperm factor appears to operate via the src/PLC $\gamma$ signaling pathway, as its action is blocked by the appropriate dominant negative $\mathrm{SH} 2$ domain construct (451).

\section{How a sperm activates an egg - an evidence based perspective}

Having set the stage, we now have to test the notions of sperm-egg activation against each other. 
The first thing to point out is that there may not be a single, universal biochemical mechanism that operates at fertilization. For example, SH2 domains inhibitory to $\mathrm{PLC} \gamma$ and src-family kinases will block fertilization in sea urchin, starfish, zebrafish and ascidian (60, $61,265,448,452,453,482)$, but not in mammals or frog $(362,363)$. Nonetheless, there is other evidence that the src/PLC $\gamma$ pathway may be central to fertilization in these species. A src-related tyrosine kinase in Xenopus co-immunoprecipitates with PLC $\gamma$ after fertilization; the association is blocked by the protein tyrosine kinase inhibitor PP1 (471). Methyl $\beta$ cyclodextrin treatment of Xenopus oocytes, intended to deplete cholesterol from lipid signalling rafts, caused a marked decrease in tyrosine kinase activity and blocked the fertilization calcium transient (470). The tyrosine kinase inhibitors lavedustinA and tyrphostin B46 prevented the fertilization calcium wave, as did a 20 amino acid truncation of the src SH2 domain (178), inhibitions overcome by calcium injections. In rat oocytes, the tyrosine kinase inhibitors PP2 and SU 6656 blocked resumption of meiosis, but not other manifestations of the fertilization response (549). If there is a consensus, it is that activity of one or other phospholipase $\mathrm{C}$ may be the common element $(452,474)$. To test this consensus, we shall have to examine the suggestions that cGMP, nitric oxide or NAADP may be the activating messengers at fertilization in echinoderms.

The suggestions around NO and cGMP are based on the observations that both NO and cGMP can trigger a calcium transient in sea urchin eggs and that both increase early during fertilization. Cyclic GMP has been measured in egg suspensions and shown to rise by $20 \mathrm{~s}$ after insemination, the earliest time point measured $(90,293)$. The timing in egg populations can be related to timings measured in single eggs by convolving the fertilization rate with the known kinetics of the latent period and the rise of the fertilization calcium transient (94). The peak of the calcium transients in an egg population (that is, the time at which the maximum number of eggs are undergoing a calcium transient) occurs at $30 \mathrm{~s}$ after insemination; however, since the latent period varies considerably in length from one egg to another, as many as $95 \%$ of eggs in a population will have initiated a calcium signal by $15 \mathrm{~s}$ after insemination (94). From timing alone, it is difficult to argue that any increase in cGMP precedes the calcium transient. Peak concentrations of cGMP have been measured as 20-100 $\mathrm{nM}(90,293)$. A cytoplasmic concentration of around $5-10 \mu \mathrm{M}$ is required to activate an egg after microinjection $(293,601)$. This is a large discrepancy. By contrast, $\mathrm{InsP}_{3}$ concentrations reach 0.2-0.3 $\mu \mathrm{M}$ at their peak at 20s after insemination (293) and eggs activate at cytoplasmic concentrations of $2 \mathrm{nM}$ (605). The only known target of cGMP is the G-kinase, which can be inhibited by RcAMPS. At concentrations demonstrated to block cGMP-induced calcium release in both sea urchin homogenates and intact eggs, inhibitors of this pathway do not prevent the initiation of the fertilization calcium transient $(164,312$, 316). On these grounds, I think it unlikely that cGMP is an initiating messenger at fertilization.

The early increase in nitric oxide at fertilization (292) was not detected in a subsequent study(301) in which the initiation of the calcium transient was measured simultaneously using fluorescence imaging. The later study was unable to reproduce the supporting observation that oxyhemoglobin, the NO scavenger, blocked the initiation of the fertilization calcium transient. It is clear that NO activates eggs by stimulating the cGMP/cADPr pathway (617) and it is known that blocking the later elements of this pathway does not prevent the fertilization calcium transient from occurring $(164,301,312,316)$ and that the NO pathway does not operate at fertilization in ascidian or mouse eggs(221). It is logical therefore to circumvent this anomaly in sea urchin by suggesting that NO may act through a nitrosylation mechanism (292), though there is no evidence that such a pathway exists in eggs. It is equally logical to suggest that since activation of eggs by NO (but not fertilization) is blocked completely by cGMP/cADPr antagonists this pathway cannot be present. 
The suggestion that NAADP may be the activating messenger is also based on the observation that NAADP triggers calcium release in unfertilized eggs $(86,307,465)$ and that NAADP is found at activating concentrations in sperm $(45,86)$. However, inactivating the NAADP pathway does not prevent the initiation of the fertilization calcium wave in sea urchin eggs(86). Instead it inhibits the calcium currents of the fertilization action and activation potentials $(86,380)$. Nonetheless, downregulation of $\mathrm{InsP}_{3}$ signalling in starfish does not prevent initation of the fertilization calcium wave(329), implying that in starfish NAADP is an activating messenger. Set against this observation is the finding that microinjection of a a protein domain that binds $\mathrm{InsP}_{3}$ with very high affinity completely abolishes the fertilization calcium transient at fertilization in starfish (227), as does microinjection of dominant negative SH2 domains that antagonize src kinase and PLC $\gamma(61$, $174)$, though it should be acknowledged that these protein domains were inhibitory only at high concentration.

On balance, my view is that in general the consensus holds and that PLC activation is central to the initiation of the fertilization calcium transient. In echinoderms and ascidian, the PLC is likely PLC $\gamma$, activated by a src-like kinase (452); in mammals the PLC is likely PLC (474). It is conceivable that an integrin receptor mechanism may operate in frog oocytes at fertilization(226). The NAADP pathway constitutes an important component of the fertilization response in several species in modulating membrane potential through activation or inactivation of calcium channels $(13,86,380)$ and in contributing to postfertilization calcium oscillations in ascidians(13). cADPr and RyR are important at the egg cortex in controlling cortical granule exocytosis $(13,14,22)$ and contribute to the calcium wave in sea urchin and $\operatorname{starfish}(164,458)$.

The major signalling pathways at fertilization are depicted in Figure 5. These conclusions by default favour the sperm-egg fusion model of egg activation.

\section{E. What we mean by egg activation}

A fertilized egg differs from an unfertilized egg and the change is irreversible. In this sense as we noted in the introduction the calcium signal changes the cell state. The calcium signal is necessary and sufficient to bring about most of the changes that occur $(512,513)$. Calcium ionophore can trigger the transient and produce parthenotes: this demonstration was in a sense the culmination of the debate between the materialists and the vitalists. Calcium alone though is not quite enough. The sperm's other role besides triggering the fertilization calcium transient and providing half the genome is to supply centrosomes $(50,431)$. The centrosome is increasingly thought of as the organelle of the cell division cycle, providing the duality needed to split the cell $(140,475,476)$. This is a reminder that fertilization is above all a cell cycle event. It is straightforward to separate those events at fertilization that are peculiar to fertilization and those that are part of the general framework of cell cycle regulation.

In the first group of events lie sperm incorporation (199), the exocytosis of cortical granules $(13,14,32,55,56,62,101,133,231,270,271,275,359,393,408,437,450,467,546$, $606)$, the elongation of microvilli $(31,185,350,589)$, the production of superoxide (205, $206,426,427,570)$ and the reactivation of metabolism $(141,543,619)$. Cortical granule exocytosis, for example, is responsible for elevation of the fertilization envelope and for the zona reaction, both designed to prevent interaction with supernumerary sperm. These events are triggered directly by the ionic changes at fertilization $(33,465,592,608)$.

The second group of events involve the cell cycle control proteins in the relief of the cell cycle arrest of unfertilized eggs and oocytes. 
Eggs and oocytes of different species are arrested at different points in the cell cycle. All germ cells produce secondary oocytes that must undergo a process of maturation in order to be fertilized. Maturation, among other things, requires progression through meiosis and meiotic recombination (602). Species differ in their point of arrest during meiotic maturation as they await fertilization. Ascidian oocytes, for example, arrest during first meiotic metaphase after recombinantion but before separation of homologous chromatids and extrusion of the first polar body. Mammalian and frog oocytes arrest in second meiotic metaphase while sea urchin eggs have completed meiosis and are arrested in G1 of the first post-meiotic cell cycle. Meiotic metaphase arrest is maintained by sustained activity of the mitotic kinase, cdk1/cyclin which maintains chromatin in its condensed state and stabilises the meiotic spindle (602); the interphase arrest in sea urchin is maintained by suppression of cyclin synthesis by a cytoplasmic $\mathrm{pH} 0.5$ units more acidic than that of fertilized eggs (145, $608,619)$. From the perspective of the division cycle, egg activation is the breaking of this stasis. The major role of the fertilization calcium signal is to regulate cdk1 and cyclin.

A distinguishing feature of the cyclins is that they are synthesized during interphase and destroyed abruptly during mitosis(220). It was this feature that led to their initial discovery in sea urchin eggs(145) as a by-product of experiments that were aimed at understanding why protein synthesis was rapidly turned on after fertilization. The rate of protein synthesis is markedly $\mathrm{pH}$ dependent in sea urchin eggs and homogenates $(186,619)$ and can, for example be stimulated by weak bases that alkalinize the egg cytoplasm to levels comparable to those measured after fertilization (186). The rapid alkalinization after fertilization is achieved by activation of a $\mathrm{Na} / \mathrm{H}$ antiporter(241). The antiporter is activated by protein kinase $\mathrm{C}$, which in turn is stimulated by both the fertilization calcium transient and by the diacylglycerol produced by the activation of phospholipase $C(138,541)$. There are echos in this mechanism of the events that occur when quiescent somatic cells are stimulated to enter the cell division cycle and proliferate by growth factors $(210,583)$, but there are major differences. For example, events in somatic cells include sequential transcription of cell cycle control genes, while in the sea urchin egg, cell cycle gene products are already present stored as maternal proteins or mRNA $(294,390,478,531)$.

In oocytes that are arrested in meiosis awaiting fertilization, a cell cycle stage that is usually very transient is preserved as it were in aspic. Metaphase is a crucial phase of cell division, when the paired chromosomes line up just before they are separated at anaphase into each daughter cell. Correct alignment on the spindle is essential, as mis-segregation of chromosomes can cause both apoptosis and cell transformation due to the unmasking of recessive alleles and alterations in gene dosage. Perhaps unsurprisingly, cell cycle control includes a so-called checkpoint mechanism at metaphase that keeps unattached chromosomes under surveillance, not allowing anaphase onset until all are safely anchored to kinetochore microtubules (76). The oocyte protein mos appears to hijack this checkpoint mechanism to maintain a metaphase arrest before fertilization $(569,580)$, preventing cyclin degradation. The fertilization calcium signal does not immediately interact with mos signalling $(335,336)$. Instead, it bypasses the checkpoint by stimulating cyclin degradation via CaMKinaseII-mediated stimulation of cyclin ubiquitination $(336,441)$ and stimulation of the proteasome degradation machinery $(11,252)$. The major role of calcium in frog and mouse eggs at fertilization is to re-initiate anaphase onset by activating the anaphase promoting complex.cyclosome (APC/C), relieving inhibition of cyclin degradation and stimulating proteasome activity to allow the cell cycle proceed.

Unlike sea urchin eggs, mature mammalian oocytes continue to undertake protein synthesis as they wait to be fertilized. In fact, transcription inhibitors like cycloheximide will relieve the metaphase arrest when added to unfertilized mammalian eggs (392), demonstrating that continuing cyclin synthesis is essential for the maintenance of the meiotic arrest. This 
observation is significant, as it bears on the question of the function of the repetitive calcium transients that are a characteristic feature of mammalian fertilization. It was first observed that a single calcium transient could relieve the metaphase arrest of mammalian oocytes $(513,582)$; this left the function of the succeeding transients uncertain. Moreover, it was shown that these subsequent transients coud be blocked by injection of the calcium chelator BAPTA without obvious effect(582). Protein synthesis is the key to understanding this paradox. A single calcium transient is effective only in ageing oocytes, where protein synthetic capacity is reduced. It has also been found that BAPTA itself blocks protein synthesis(298). There is a good correlation between the number of repetitive calcium spikes and cell cycle progression in mouse oocytes $(79,417,539)$. A clear demonstration of the importance of successive calcium spikes has been made using a cyclin B-GFP fusion protein in asidian oocytes $(321,401)$.

It was first shown in ascidian oocytes that the repetitive calcium spikes after fertilization correlate well with episodes of cyclin destruction, as measured by the loss of cyclin B-GFP fluorescence $(321,356)$. The same is true in mouse $(401,402)$. Calcium spike frequency in both mouse and ascidian is linked to cyclin levels $(321,402)$, implying that cdk $1 /$ cyclinB kinase levels control the spiking machinery (356). The inference is that the continuing synthesis of cyclin in mouse and ascidian requires multiple calcium spikes to bring cyclin levels down sufficiently to inactivate the $\mathrm{cdk} 1 /$ cyclin B kinase and permit exit from meiosis. This may be a sufficient explanation for the existence of multiple calcium spikes after fertilization.

There may be more to it, however. Using a complex and ingenious machine that by application to mouse or rabbit oocytes of high electrical field strengths in low ionic strength calcium-free media followed by re-addition of calcium-containing media is able to produce a simulacrum of the repetitive fertilization calcium pulses, it has been shown that the duration and frequency of calcium pulses determines the extent of both early development(417), the inactivation of Cdk1, MAP kinase and the recruitment of maternal mRNA (126) and of implantation rate and post-implantation development(416). The molecular mechanisms responsible for these effects are undiscovered, though it has been shown that CaM Kinase II activation can integrate repetitive calcium pulses $(28,348)$ and that the frequency of repetitive calcium pulses can regulate the extent of gene expression (326). It has also been demonstrated that the very calcium spikes that initiate the developmental programme in normal mouse oocytes lead to the induction of the cell death programme in aged oocytes $(180,181)$.

A key consequence of repetitive calcium spiking is the episodic stimulation of mitochondrial metabolism and the enhanced production of ATP. Measurements of mitochondrial autofluorescence due to NADH and flavoprotein redox changes show increases in step with the calcium spikes $(128,129)$. In ascidian oocytes, each calcium spike is accompanied by increased oxygen consumption and inhibition of mitochondrial respiration inhibits the spikes (128). It is striking that supernumerary spikes can be elicited by local uncaging of ATP (128). The same correlation between mitochondrial autofluorescence and calcium spikes is seen in mouse oocytes after fertilization (129) and again mitochondrial respiration was essential for spiking activity. These finding echo data from somatic cells that demonstrate that calcium uptake into mitochondria regulates their respiration $(106,118,444)$. The stimulation of metabolism by the calcium spikes may be one explanation for the need of multiple spikes for optimal development, as uncovered by Ozil's studies $(126,416)$.

A key role of the fertilization calcium signal is thus to restart the cell cycle, via calmodulin/ CaM KinaseII pathways that interact with the cell cycle control machinery. In mammals and 
ascidian, full activation at fertilization requires repetitive calcium spikes. It is worth asking what controls this repetitive calcium spiking activity.

\section{F. The mechanism of repetitive calcium spiking at fertilization}

Most calcium signals that rely on calcium release from intracellular store, however generated, have an oscillatory component (40). Both the InsP $\mathrm{P}_{3}$ - and ryanodine-receptors have both positive and negative feedback properties that may favour calcium oscillations. Both show CICR, where increased cytoplasmic calcium concentrations enhance the open probability of the channels and both have bell-shaped responses to cytoplasmic calcium: at higher concentrations, the effect of a further increase is inhibitory(132). In themselves, these characteristics are sufficient to generate oscillatory calcium release; in addition, the depletion of ER calcium adds a further negative feedback element(40), while, for the $\mathrm{InsP}_{3}$ receptor, additional positive feedback can come from calcium-stimulated hydrolysis of PtdInsP $P_{2}$. As we have seen, both ascidian and mammalian oocytes undergo repetitive cytoplasmic calcium spikes after fertilization. Their detail is distinct: in ascidian, the spikes immediately after fertilization are superimposed on a larger sustained transient (358), whereas in mammals, each spike is separate and the interval between spikes can last for several minutes(539). The second phase of spikes in ascidian more closely resembles that in mammalian oocytes. Nonetheless, both spiking patterns can be mimicked reasonably well by slow infusion or release of $\mathrm{InsP}_{3}$ into the oocytes (13, 14, 150-152, 166, 247, 358, 373, 375, $535,547)$, suggesting that the spiking pattern may be governed by the basic feedback properties of the $\mathrm{InsP}_{3}$-receptor(9). This idea sits well with our earlier analysis of fertilization signal transduction mechanisms that point to $\mathrm{InsP}_{3}$ as the activating messenger.

We have, then, an explanation of why the calcium spikes start, but why do they stop? The answer lies not in the calcium release receptors themselves, but with the cell cycle kinase. The calcium spikes can be sustained indefinitely, or at least for as long as people have had the patience to look, by preventing exit from meiosis. A crude way to achieve this is to use microtubule inhibitors, for example colcemid, to invoke the metaphase checkpoint in fertilized eggs and maintain a metaphase state (279). A more elegant and informative approach has been to use exogenous cyclin B or non-degradable cyclin B to defeat the APC/ proteasome and maintain metaphase levels of cdk1/cycin B kinase activity $(321,402)$. The simple conclusion from these experiments is that the mitotic kinase maintains the oscillations by, for example, stimulating the continued production of $\mathrm{InsP}_{3}$, sensitizing the $\mathrm{InsP}_{3}$ receptor itself or maintaining high levels of ER calcium by stimulating the SERCA pump. The true state of affairs may be more interesting.

There is evidence from experiments in mammalian eggs that the oscillations are driven by a factor sequestered in the zygote nucleus. Transfer of the interphase nucleus of the one-celled embryo to an unfertilized oocyte can activate it: the nucleus breaks down in response to the elevated cdk/cyclin B kinase activity and the initiation of the calcium spikes correlates with breakdown of the nuclear envelope (278). Analysis of the cessation of spikes in fertilized eggs shows that they cease at the time of reformation of the pronucleus; inhibiting reformation of the nuclear envelope with a lectin sustains the calcium spikes, not withstanding that $\mathrm{cdk} 1 / \mathrm{cyclin} \mathrm{B}$ kinase activity falls to the same levels as controls(346). This is good evidence that it is not the fall in cdk1/cyclin B kinase activity per se that leads to cessation of the calcium spiking activity, but the formation of the pronucleus. So the link between the fall in kinase activity and the ending of the train of calcium spikes is indirect and due to the need for mitotic kinase activity to fall in order that the nuclear envelope be reassembled. These data, combined with the nuclear transplantation evidence, indicate that nuclear sequestration of an activity responsible for generating and maintaining the calcium spiking causes them to cease. If the nucleus were to sequester the (PLC $\zeta$ ) activity, then the explanation of the initiation and cessation of the fertilization calcium spikes would be neat 
indeed. This has now been demonstrated $(280,297,631)$. However, it should also be borne in mind that enucleate oocytes (merogones) show calcium transients that cease at around the same time as nucleate merogones (113), so cdk activity must play some role in bringing the transients to a stop.

\section{CALCIUM AND THE EMBRYONIC CELL CYCLE}

\section{Summary}

Evidence that calcium signals control cell division in early embryos is presented. The section begins with the control of the separation of chromosomes at anaphase and moves on to cytokinesis, explaining the relative advantages of different embryos in these studies: large embryos lend themselves to the study of cytokinesis, while smaller embryos are more transparent, suiting them to studies of mitosis. The role of calcium in getting into mitosis and the few studies that have used genetics to investigate calcium signalling during cell division are discussed. It is concluded that calcium signals are essential at all three stages of cell division in early embryos. There is need for better information on how the calcium signals feed in to the protein regulatory networks that effect cell cycle transitions.

The fertilization calcium signal interacts with and modifies cell cycle control proteins. It is natural to wonder whether these sorts of interaction may take place more generally during embryonic cell cycles. The question, then, is whether control of mitotic progression by calcium is peculiar to the events of fertilization or whether fertilization is merely a special case of a mechanism that is common to all cell cycles.

Immediately after fertilization, embryos undergo a series of very rapid cell divisions. The embryos of external fertilizers show particular expedition: a sea urchin's embryos undergo 8 cycles of cell division within $4 \mathrm{hr}$, a frog's 8 cycles within $6 \mathrm{hr}$ and a fruit fly's 13 nuclear divisions within $3 \mathrm{hr}$. During these divisions the embryo relies heavily on maternal stores of metabolic substrates, mRNA and protein; there is little or no gene transcription $(112,195)$. The rapidity and simplicity of embryonic cell division cycles have attracted interest and a good deal of the knowledge we have of cell cycle control comes from experiments in early embryos. Answers to the question we have posed have come largely from experiments in sea urchin, frog, fruit fly and zebrafish embryos. Cell division involves three successive events: dissolution of the nuclear envelope, chromatin condensation and formation of the mitotic spindle; separation and segregation of the chromosomes by spindle elongation; and separation of daughter cells by the formation of the cleavage furrow. Each of these events is blocked by lithium treatment and the block rescued by addition of myo-inositol (30), a classical demonstration(38) phosphoinositide signaling is involved. Cell cycle progression is also blocked by L690,330, a bisphosphonate inhibitor of inositol monophosphatase that acts similarly to lithium (479). I will discuss the role of calcium in regulating each of these events, but not in turn.

\section{A. Separation of chromosomes - the metaphase/anaphase transition}

The events of this phase of mitosis are analogous to the events that occur during fertilization in frog, ascidian and mammal. So much so that when sperm factor is injected into fertilized eggs it induces calcium oscillations when cdk1 activity increases at mitosis (551). The most complete set of data relevant to the possible role of a calcium signal at metaphase come from sea urchin embryos. I shall also comment on such data as exists in other embryos. 
1. Sea urchin-In sea urchin embryos, the separation of sister chromatids is accompanied by a brief, sharp, small calcium transient, an order of magnitude smaller than those measured at fertilization(188). The transient occurs 1-2 min before spindle elongation and always precedes it. Sea urchin embryos can divide normally in sea water lacking calcium, so the inference is that the transient is due to release from internal stores. The transient can be blocked using the calcium chelator dibromo-BAPTA or the $\mathrm{InsP}_{3}$-receptor antagonist heparin, by microinjecting them once the spindle has formed. Blocking the calcium transient prevents separation of sister chromatids. It is possible with photolysis to reverse this effect: Uncaging calcium from NP-EGTA using a UV pulse or uncaging $\operatorname{InsP}_{3}$ in the presence of heparin leads to normal chromatin segregation(188). These data indicate that the metaphase calcium transient controls separation of sister chromatids.

The chromosomes of heparin-injected embryos underwent chromatin decondensation and a single nucleus was formed(188), indicating that cyclin destruction by the APC was independent of the calcium signal and a situation clearly different from what happens in mouse eggs after fertilization (see above). Despite the inferred fall in cdk1/cyclin B kinase activity, the cleavage furrow, though induced, regressed in most cases(188).

2. Drosophila-Fruit fly embryos undergo 13 syncytial nuclear divisions whose control mechanisms are very similar to those of other embryos, with the exception that cyclin B is degraded only locally in the region of the spindle; cytoplasmic cyclin B is spared. Calcium signals accompany mitosis in syncytial Drosophila embryos (Figure 6). It might be conjectured that a metaphase calcium signal, if it exist, might be restricted to the spindle region. This is the case (422). In Drosophila, microinjection of a calcium chelator into the perivitelline space surrounding the embryo arrests the nuclear division cycle and if the microinjection is done just before the embryos enter mitosis, nuclei arrest with their chromatids arranged as at metaphase(422). A similar result is obtained using $\mathrm{InsP}_{3}$ antagonists: the embryos have a Ins $\mathrm{P}_{3}$-sensitive calcium store. Two antagonists were used, one the InsP $\mathrm{P}_{3}$ sponge, a recombinant protein consisting of the $\mathrm{InsP}_{3}$ binding domain of the type $1 \mathrm{InsP}_{3}$-receptor (584), the other $\mathrm{p} 130$, an inactive phospholipase that binds $\mathrm{InsP}_{3}$ (548). The former has a point mutated control that has a much reduced affinity for $\mathrm{InsP}_{3}$ which was without effect. Thus, just as in the sea urchin, it looks as though a metaphase calcium signal is required for separation of chromatids.

3. Xenopus-Frog eggs are highly pigmented and it is impossible to gain sight of the mitotic spindle in living eggs. It has been shown that microinjection of heparin (200) and an InsP $\mathrm{P}_{3} \mathrm{R}$ antibody(395) prevent division of the one-celled embryo and EGTA and dibromoBAPTA to prevent division in the two-celled embryo (403), so while in broad terms it appears that the $\mathrm{InsP}_{3}$ signalling system is essential for cell division in the frog embryo, it is unclear which stage of mitosis is affected by these calcium antagonists.

4. Zebrafish-Individual blastomeres of zebrafish embryos show striking cacium transients as the embryo develops from the 32 to the 1000-cell stage (Figure 7 and (442)), but there are no clear temporal correlations between the transients and cell division, unless embryos are made to express an ectopic pattern gene, XWnt5A(496). We shall return to this observation below.

\section{B. Separation of daughter cells - cytokinesis}

Embryos come in all shapes and sizes. As a consequence, the topography of cytokinesis varies during the very early blastomere divisions. In the smaller $(0.1 \mathrm{~mm})$ embryos of echinoderms and mammals, the holoblastic cleavage furrow is rotationally-symmetric and resembles the cleavage furrow of dividing somatic cells(438); in the large (1 mm) embryos 
of frog and fish, the cleavage furrow initiates at the animal pole and moves circumferentially along a line of longitude as well as progressing inwards through the cytoplasm(492); the fly embryo has no cleavage furrow at all during the first 13 nuclear divisions, though it does have actin-based movements of plasma membrane around and between dividing nuclei that can be thought of as half-furrows and known as meroblastic cleavage. Despite these differences in topography, evolution and scale, all furrows share the property that their location and progression are dictated by the position of the mitotic spindle. The location of the mitotic spindle in mid-anaphase (in the sea urchin, precisely $3 \mathrm{~min}$ after anaphase onset(493) determines the point of constriction and membrane addition(492). It is reasonable to suppose that calcium is involved in controlling the evolution of the furrow during cytokinesis, since it is known to regulate both acto-myosin based motility (396) and addition of membrane through granule fusion in both embryos and somatic cells $(43,44,511,515$, 562). Size matters. It has been easier to image cytoplasmic calcium during large meroblastic cleavages and I shall discuss these first.

1. Frog and Fish-Very soon after fertilization of fish eggs, the cytoplasm segregates to a hemispherical cap atop the egg. The first cleavage furrow bisects the hemisphere, originating at the apex, spreading laterally and deepening as it goes. The size of fish eggs suits them to luminescence imaging. The calcium sensitive photoprotein aequorin detects a sustained calcium signal in medaka eggs that is located at the base of the furrow and precedes the furrow as it deepens(156); this observation established the concept of slow calcium waves, the idea being that calcium release is triggered by the mechanical forces generated by the advancing furrow. The wave-like nature arises because calcium is thought to stimulate continuing force generation by the actomyosin contractile ring as it tightens (156). A similar pattern of calcium was observed at better spatial resolution using ratiometric confocal calcium imaging in both zebrafish(74) and frog(395) embryos, where it was clear that a slow calcium wave propagated laterally along the furrow arc. It was also established that the signal depended on release of calcium from internal stores by $\operatorname{InsP}_{3}(74$, 395). Using a more sensitive aequorin, the distinction between the slow calcium wave accompanying lateral extension of the furrow and that driving furrow deepening was very evident $(103,594)$.

Subsequently, a third calcium signal that precedes initiation of furrowing has been observed (73) that is suggested to be involved in positioning the furrow. This study offers a good summary of the work in fish embryos, showing that, as before, furrowing can be blocked by BAPTA and heparin and in addition that they cause regression of already-established furrows and that BAPTA microinjection causes the dissolution on the contractile band. It also sets out clearly the three phases of the calcium signals observed during cleavage of fish embryos: calcium spikes that precede cleavage by several minutes, the slow wave accompanying lateral extension of the furrow and the signal that accompanies furrow deepening, which in this case was seen as mutiple local calcium transients. Neither lateral extension of the furrow nor furrow deepening was affected by antagonists of the RyR and NAADP calcium signaling pathways (315). Extension was unaffected by the InsP $\mathrm{P}_{3}$ antagonist heparin, while deepening was; the reverse was the case for the store operated calcium channel antagonist 2-APB (315). These observations suggest that extension may require store-operated calcium entry, while deepening requires $\mathrm{InsP}_{3}$-induced calcium release, though the specificity of 2-APB in particular is questionable. No furrow initiation calcium transient has been observed in Xenopus embryos, and transients considered by Miller (315) to be equivalent to the lateral extension transients and deepening transients in zebrafish are observed to occur after cytokinesis when the cleavage furrow is imaged using fluorescent WGA(403). It has also proved possible to abolish these two transients without grossly perturbing cytokinesis, leading to the suggestion that in Xenopus the two transients are associated, respectively, with new membrane formation in the furrow and gap junction 
formation between blastomeres (403). This last study is at odds with all the other recent observations linking calcium to regulation of cytokinesis.

The last word goes to an imaginative set of experiments in which cerebellar microsomes, rich in type $1 \mathrm{InsP}_{3} \mathrm{R}$, were shown to induce a supernumerary furrow in a newt embryo during second cleavage at their site of injection (372). The site of injection was chosen to be at the embryo equator and the extra furrow formed at the time that the native furrow reached the equator in its journey to the vegetal pole, some 40-50 min after injection of the microsomes. This offers good evidence that the extra furrow was under the control of endogenous cytokinetic mechanisms. The extra furrow formation was inhibited by the $\mathrm{InsP}_{3} \mathrm{R}$ antagonist heparin and by a monoclonal antibody known to block the Ins $\mathrm{P}_{3} \mathrm{R}$. Microsomes from mice lacking the type $1 \mathrm{InsP}_{3} \mathrm{R}$ were much less effective than those from wild type mice. These experiments suggest a role for ER and $\mathrm{InsP}_{3}$ in Xenopus, confirming the data from zebrafish and the observation that ER is found close to the site of furrow extension (315).

Imaging the cleavage furrow has thus offered good evidence that local calcium signals specify furrow formation and progression and has cleared up some of the confusion from whole cell measurements using aequorin and calcium sensitive electrodes that have suggested either that calcium in Xenopus embryos is either high $(187)$ or low $(254,283)$ at cleavage. In the light of the imaging data, it seems most likely that global intracellular calcium increases as mitosis approaches $(254,283)$, but that local calcium signals specify the cleavage process itself.

The size of fish and frog embryos offers us our clearest picture of the calcium signals involved in cytokinesis. It is reported that as the large blastomeres give way to smaller as cell division progresses, the different phases of calcium signalling become indistinguishable: not surprising, then, that no comparable detail has been resolved in echinoderm and mammalian embryos or in somatic cells(209).

2. Echinoderm and mammalian embryos-Cleavage of echinoderm and mammalian embryos does not require furrow extension, as the furrow propagates by deepening alone. Local calcium increases and calmodulin activation beneath and around the cleavage furrow have been observed $(188,525,566,612)$ and ER accumulates in the cleavage furrow in human early embryos (182), but there is as yet no systematic study of calcium and cleavage. Indeed, earlier work in mammalian embryos was unable to detect cleavage-related calcium signals (564).

3. Drosophila embryos-A prominent calcium signal accompanies the unusual half cleavages of syncytial Drosophila embryos. Though all nuclei share a common cytoplasm, once they have migrated to the embryo cortex each sits within a plasma membrane cup that is organized by the mitotic spindle and actomyosin cytoskeleton, an arrangement reminiscent of cytokinesis . Very superficial calcium increases closely correlated with actin dynamics in space and time occur as the cups contract and extend between nuclear divisions(422). Because nuclear divisions are asynchronous being most advanced at the poles, the cortical calcium increase takes the form of a slow wave passing from both poles towards the embryo equator. The signal is extinguished by chelating external calcium or by $\mathrm{InsP}_{3}$ antagonists(422).

4. Targets of cleavage calcium signals-The suggestion is that calcium signals may be involved in all three phases of cytokinesis: induction of the furrow, assembly of and force generation by the contractile ring and membrane addition and maintenance of the ring as the furrow deepens. For the two last, the evidence is reasonably clear. 
The contractile ring is an actomyosin based motility system(259). In smooth muscle, contraction is regulated by calcium via camodulin activation of myosin light chain kinase (MLCK), an event that both assembles myosin into short filaments and permits forcegenerating crossbridge recycling. In somatic cells, GFP- calmodulin localizes to the cleavage furrow and myosin is known to be phosphorylated at the site favoured by MLCK. It has been shown in sea urchin embryos that calmodulin is activated at the cleavage furrow and that cleavage is inhibited by an anti-MLCK peptide $(566,612)$ (Figure 8).

Membrane addition by regulated exocytosis is known to be a calcium-dependent event. More generally, it has been elegantly shown in both sea urchin embryos and somatic cells that membrane addition as a consequence of injury to the plasma membrane is due to calcium-triggered membrane fusion with properties very similar to those of exocytosis and that requires both microtubule and actin-based translocation of vesicles to the plasma membrane $(561,562)$. It is highly likely that this mechanism operates to add membrane to the furrow during cleavage(492), though this has not yet been demonstrated.

Whether calcium is directly involved in positioning the cleavage furrow is less certain. The position of the furrow is known to be determined by the disposition of the spindle's astral microtubules(493), even when cleavage is asymmetric(249) or markedly perturbed(438); it appears that the furrow forms in the region of lowest microtubule density(634). The mechanism is unclear, but can be modelled formally as if the causal agent were diffusing from regions of high microtubule normal surface density to regions of lower density(634). There are no clues pointing to calcium here. What of the calcium spikes that are seen in fish embryos minutes before cleavage begins? They may represent cleavage positioning signals $(73,103,337)$, but may also be signals controlling events earlier in mitosis (103). There is a reciprocity between the observations in the large and small embryos. Cleavage calcium signals are readily observed in large embryos, but the events of mitosis at the mitotic spindle cannot easily be seen; in small embryos, nuclear and spindle calcium signals have been imaged, but the cleavage signals are less readily separable into their components. It is very likely that the spiking that precedes cleavage in zebrafish represents the anaphase calcium signal, for example. I've already hinted, too, that the effects of inhibitors on cleavage furrow formation may not be straightforwardly interpretable. For example, blocking the anaphase calcium transient in the sea urchin embryo with heparin prevents chromatid disjunction, as we have seen, but also induces regression of the cleavage furrow(188). Is this because a cleavage calcium transient is blocked(188) or because mitosis itself is aberrant? What is evident is that blocking these calcium signals in the sea urchin embryo does not prevent cleavage furrow initiation. A role for calcium as an initiation signal is, to me, unlikely, though microtubules emanating from the spindle midzone may position endoplasmic reticulum to provide a calcium signal that stimulates ingression of the cleavage furrow ( 314 , $315)$.

\section{Getting into mitosis - nuclear envelope breakdown and chromatin condensation}

The demonstration that a calcium signal controls entry into mitosis was the first indication that calcium was a cog in the cell cycle control machinery. A small transient was detected in sea urchin embryos using the fluorescent calcium indicator fura2, then a relatively novel indicator dye (434). It preceded nuclear envelope breakdown (NEB) by a few minutes. The causal element of this relationship was proven by the observations that microinjection of calcium or InsP $\mathrm{P}_{3}$ would induce NEB precociously $(510,573)$ and that calcium chelators would block NEB. Reversible inhibition was demonstrated using a caged calcium chelator, NP-EGTA (613). NEB in starfish embryos is preceded by a calcium spike and blocked by heparin injection(525). In sea urchin embryos, the NEB calcium transient was reported to be localized to a perinuclear region (613) and in Drosophila analysis of the nuclear region also revealed an NEB transient (422). Calcium oscillations are observed in mouse embryos just 
before NEB (279) and are reported to be dependent on calcium mobilization (564). Others, however, have failed to find evidence of an NEB-linked calcium transient $(113,179,551$, 564) and the authors of the original observation in the mouse (279) have subsequently reported that calcium signals are seen only once NEB has occurred (346).

\section{Genetic approaches to calcium signaling in early embryos $-C$. elegans}

Calcium signals and calcium signaling messengers are ubiquitous and calcium signaling mutant phenotypes are thus highly pleiotropic. Studies in $C$. elegans illustrate the difficulties of a genetics approach to calcium signaling but also offer an example of how a transgenic approach can give clues to the outcomes of calcium signaling in embryos. C. elegans possesses a single $\mathrm{InsP}_{3} \mathrm{R}$ gene, itr 1 , and the receptors are expressed in a wide range of tissues, including gonad, nervous system, pharynx and intestine (29). However, itr-1 mutants are sterile, due to an ovulation defect (95), precluding an analysis of the lack of InsP $\mathrm{P}_{3} \mathrm{R}$ during early development. An alternative approach has been developed by expressing the $\mathrm{InsP}_{3}$ binding domain of the $\mathrm{InsP}_{3} \mathrm{R}$ under control of a heat shock promoter (584). The binding domain competes for InsP $\mathrm{P}_{3}$ with the endogenous $\mathrm{InsP}_{3} \mathrm{R}$ and disrupts InsP $\mathrm{P}_{3}$ signalling. It is known as an $\mathrm{InsP}_{3}$ sponge. Expression of the sponge severely disrupts embryonic development. The first cleavage division is affected, as is gastrulation as a consequence of a failure of gut precursor cell differentiation (584). This suggestion that $\mathrm{InsP}_{3}$ signalling may be involved in early cleavage is supported by the observation that a weak mutant allele of itr- 1 causes a failure in completion of cleavage in the first cell cycle (507). An increase in cytoplasmic free calcium measured with calcium green has been reported at the onset of mitosis and in the cleavage furrow during the first cell division (67). A calcineurin loss of function mutant shows defects in fertility and egg laying very similar to those of a gain of function CaMKII mutation, but these phenotypes appear to be related to muscle function rather than the embryo itself (26).

The pickings from genetics are slim. Even the transgenic approach with the $\mathrm{InsP}_{3}$ sponge suffers from the limitation that heat-shock induced expression takes place over tens of minutes to hours, with a similar time course for disappearance of the sponge once the heat shock is removed. The $\mathrm{InsP}_{3}$ sponge is a very promising and specific reagent for disrupting $\mathrm{Ins}_{3}$ signalling, but to properly test the role of $\mathrm{InsP}_{3}$ in specific cell cycle events, it will be preferable to microinject in vitro-expressed protein.

\section{E. The provenance and targets of embryonic cell cycle calcium signals}

Where do they come from? In general, calcium signals are generated by plasma membrane signal transduction mechanisms, but while such mechanisms exist in sea urchin embryos, for example $(25,202)$, there is a strong supposition that cell cycle calcium signals are generated endogenously as part of the cell cycle control machinery. In sea urchin embryos, levels of $\mathrm{Ins}_{3}$ rise just before NEB, at metaphase/anaphase and again at cleavage (93); progression through NEB, metaphase and cleavage is blocked by the same PLC $\gamma$ SH 2 domain antagonist that prevents fertilization(482). The question then seems to be how PLC $\gamma$ might be activated. We have no answers, other than that inhibition of MAP kinase appears to prevent the NEB transient while inhibition of the cdk1/cyclin B kinase does not(429) and, you will recall, the notion that in mouse embryos, the dissolution of the nuclear envelope may release the oscillogen PLCS (278).

In Xenopus, cell cycle calcium signals that correlate with mitosis in control embryos persist after the cell cycle is blocked with colchicine to prevent mitosis $(254,283)$. This finding implies that the calcium signaling system is a primary oscillator. 
What do they do? Well, again, data are scant. Almost certainly, calmodulin is the immediate target. A novel fluorescent calmodulin probe senses calmodulin activation (612) and identifies perinuclear activation of calmodulin in sea urchin embryos just before NEB and at the spindle poles just before metaphase (566) (Figure 8). It is possible that this activation maps to the centrosomes. A peptide based on the MLCK recognition motif can block both NEB (566) and anaphase onset. Peptide and antibody inhibitors of CaMKinaseII prevent NEB in sea urchin embryos (23). It is possible that this reflects the phosphorylation of the cdk1/cyclin B regulator cdc25 (423). Other potential targets of CaMKinase II include the proteasome $(11,252)$ and the APC activator Emi (441).

\section{A NATURAL BREAK}

Thus far, we have discussed calcium signals in eggs and embryos for which there is good cumulative evidence of causal significance as regulatory signals in the early developmental programme. Even here, we must qualify the assertion, as the apparent absence of clear evidence of calcium regulation of mitotic progression in mouse embryos gives pause for thought. Hereon in this review, the evidence for the causal significance of calcium signals to the developmental programme is much more open to debate. From this point, the rhetoric will be aiming to persuade where appropriate.

\section{CALCIUM AND THE MEIOTIC CELL CYCLE OF OOCYTES}

\section{Summary}

The evidence that phosphoinoisitide signalling pathways control the resumption of meiosis during oocyte maturation is reviewed and it is concluded on balance that the evidence points to a need for phosphoinositide/calcium signalling during resumption of meiosis. Changes to the calcium signalling machinery that occur during meiosis that enable the production of a calcium wave in the mature oocyte when it is fertilized are described.

As an oocyte waits to be fertilized, it is stopping for the second time. While the cells of the female germ line differentiate and grow in the ovary, they pause within the meiotic cell cycle (602). The meiotic cell cycle usually resumes as the oocytes are ovulated. The point at which oocytes first stop their cell cycle does not vary from species to species, in contrast to their second stopping point before fertilization. They first arrest late in interphase with an intact nuclear envelope, surrounding what in immature oocytes is known as the germinal vesicle, in which genetic recombination occurs. Just as in mitotic cell cycles, the activity of nuclear envelope, chromatin and spindle is controlled by the cdk1/cyclin B kinase, whose activity rises as germinal vesicle breakdown (GVBD) occurs. Indeed, the existence of a factor that when transferred from mature oocytes to immature oocytes led to $\operatorname{GVBD}(266$, $267,344)$ was one of the key observations that led to the discovery of the cdk/cyclin kinases(345). In addition, MAP kinase plays a key role during meiosis: it maintains chromatin condensed during the pseudo-interphase that separates the two meiotic divisions $(579,580)$. In this way, DNA synthesis is suppressed, allowing the creation of the mature, haploid oocyte.

\section{A. Calcium signals during oocyte maturation}

Meiosis is in principle difficult to study using cell physiological approaches because it takes place within the ovary. Mouse oocytes, for example, are released from the ovary only at second meiotic metaphase $(365,367)$. Most studies must accept the caveat that what has been shown in vitro may not hold in vivo. 
While there were early suggestions that calcium signals might be responsible for the initiation of oocyte maturation on release from the ovary $(59,193)$, it came to be accepted that in frog, starfish and mammalian oocyte the signal takes the form of a hormone-induced fall in cAMP $(344,602)$. More recently, it has been shown in starfish that stimulation with the maturation hormone 1-methyladenine leads to activation of a G-protein coupled PI-3kinase pathway that acts through the Akt/PKB pathway to trigger activation of cdk1/cyclin by downregulating Myt1, a cdk1 inhibitory kinase $(212,411,455,488)$. Immature starfish oocytes have well developed calcium signaling pathways. They are responsive to $\mathrm{InsP}_{3}$, cADPr and NAADP $(328,382,408,464)$ and application of 1-methyladenine generates a calcium wave in the oocyte $(459,460)$. But it was shown some time ago that GVBD occurred quite normally in the presence of a calcium chelator and $\mathrm{InsP}_{3}$ injection failed to induce GVBD, calcium therefore being neither necessary nor sufficient, despite the occurrence of a calcium spike when hormone is added (620). More recently it was demonstrated that abrogating the $\mathrm{InsP}_{3}$ pathway using an $\mathrm{InsP}_{3}$ chelator construct had no effect on maturation in response to hormone (227). However, despite these observations, there is evidence that calcium signaling plays a part during oocyte maturation.

A key finding was that though microinjection of the calcium chelator BAPTA into the cytoplasm did not block maturation, as judged by the breakdown of the germinal vesicle (GVBD), microinjection of the chelator into the GV itself was effective (463). The same report showed that a small $(100 \mathrm{nM})$ calcium increase occurred in the GV, even after cytoplasmic injection of BAPTA. It was then shown that microinjection of 50 or $250 \mu \mathrm{M}$ $\mathrm{InsP}_{3}$ or $250 \mu \mathrm{M}$ cADPr into the GV induced calcium transients and oscillations within the GV and caused GVBD in around half the oocytes treated and that time to GVBD after hormone addition doubled after microinjection of ryanodine or ruthenium red into the GV $(460,462)$. Microinjection into the $\mathrm{GV}$ of a calmodulin antibody or a myosin light chain kinase based calmodulin inhibitory peptide completely blocked GVBD and also blocked cADPr signaling (461). It appears that calcium within the GV may be regulated differentially to that in the cytoplasm (460) as in other cell types (169). It was also reported that addition of 1-methyladenine led to two successive calcium signals. The first signal controls cytoplasmic events of maturation (458), while the second is a signal local to the GV that occurs just before GVBD and is associated with a hormone-induced and cdk1/cyclin related increase in $\mathrm{Ins}_{3}$ receptor sensitivity that begins in the vicinity of the GV before spreading to the rest of the oocyte (460). Thus, as in the sea urchin embryo during mitosis (613), local calcium signals may induce GVBD in starfish oocytes.

Bivalve molluscs use calcium signals to induce GVBD. The surf clam oocyte is shed immature and begins to mature at fertilization. GVBD can be triggered by microinjection of $\mathrm{InsP}_{3}(47)$ or by depolarization with potassium chloride to open plasma membrane calcium channels(124); a similar mechanism operates in Barnea candida oocytes(125). This result can be thought of as confirming the rule: that calcium is a universal activator at fertilization (513). But other bivalve molluscs are stimulated to mature not by fertilization, but by a neurotransmitter hormone, 5-hydroxytryptamine (98). The hormone triggers a calcium transient and both it and release from arrest are prevented by the $\mathrm{InsP}_{3}$ antagonist heparin $(114,115)$ while GVBD can also be triggered by addition of calcium ionophore. It is not known how either this calcium signal, or indeed a fall in cAMP, might stimulate cdk/ cyclin and MAP kinase activities. In interpreting signaling data during oocyte maturation, it should be borne in mind that, unlike fertilization, hormone induced maturation occurs naturally within the ovary, not in sea water: in vitro experiments may not completely reproduce what happens in the ovary during physiological maturation $(365,367)$. 
Signalling pathways in immature sea urchin oocytes have not been studied because the eggs are released mature from the ovary, making the immature oocyte less accessible to the experimentalist.

GVBD can happen very quickly after hormone addition, but the process in frog and mammalian oocytes is slow and amenable to separate study. There are indications that the phosphoinositide signalling pathway is activated at GVBD. GVBD in mouse oocytes is delayed by the classical(39) phosphoinositide inhibitor lithium(428). The delay is rescued by either myo-inositol or $\mathrm{InsP}_{3}$. In frog oocytes, there is a transient increase in phosphoinositide turnover at time of $\operatorname{GVDB}(58)$ reminiscent of the increases measured during mitosis(93). Intracellular calcium concentrations have been measured in mouse oocytes around GVBD, where it is found that there are a series of repetitive transients on release from the follicle that cease at around the time of first meiotic metaphase $(63,64)$. As GVBD occurs, the transients become infrequent and may correlate with the events of meiosis. The oscillations are driven by $\mathrm{InsP}_{3}$; GVBD is blocked by the antagonist heparin(63). Permeant calcium chelators block GVDB in some mammalian oocytes, including mouse but are ineffective in others (reviewed in $(216,217)$, perhaps because the timing of the calcium signalling event varies relative to release from the ovary between species(216) or because the capacity of different oocytes to hydrolyse the permeant chelator to its active form varies.

\section{B. Calcium and control of the meiotic cell cycle}

On balance, the evidence points to a role for the $\mathrm{InsP}_{3}$ signalling system (and possibly cADPr) and calcium transients in controlling GVBD during the meiotic cell cycle. When meiotic events are controlled by fertilization, calcium clearly plays a central role in controlling meiotic progression, as when fertilization triggers GVDB in the surf clam oocyte(47), first meiotic anaphase in ascidian (356) and Cerebratulus oocytes(524) or second meiotic anaphase in frog mammalian oocytes (335). What of meiotic anaphase in oocytes where fertilization has already or has not yet occurred?

In ascidian oocytes, fertilization occurs at first meiotic metaphase. There is, you will recall, no chromatin decondensation between first and second meiotic metaphase; instead the second meiotic spindle reforms around the condensed chromosomes. Blocking the second set of calcium spikes that begin as the spindle reforms does not prevent assembly of the spindle but polar body formation does not occur $(358,632)$; the spindle leaves the cortex and its microtubules depolymerize (632). When starfish oocytes are fertilized around first meiotic metaphase, a calcium elevation or calcium spikes accompany second meiotic anaphase and second polar body extrusion $(525,526)$. Of the species whose oocytes are fertilized at second meiotic metaphase, only the mouse has been looked at. Calcium oscillations precede germinal vesicle breakdown (64) and removal of external calcium delays it(564).

\section{Priming the fertilization calcium wave}

Fertilization calcium transients are among the largest and longest calcium signals known, reaching an extreme in the frog egg, where calcium remains elevated above micromolar levels for ten or more minutes $(164,407,602)$. In most other circumstances, such large calcium waves are either unphysiological(446) or a symptom of pathology(77). Fertilization calcium responses have been called explosions $(233,603)$. One process that occurs during oocyte maturation is the priming of the explosive regenerative mechanism. After fertilization, eggs' calcium signalling mechanisms revert to a less explosive mode. One recondite illustration of this point is the response of sea urchin eggs activated with ammonia (608). Ammonia re-activates the cell cycle without triggering a fertilization calcium wave; instead, a comparably large transient occurs just before NEB, at the time that the ten-fold 
smaller NEB transient would normally occur. One obvious interpretation of this observation is that the charge hangs fire after ammonia treatment, being detonated only once another fuse is lit by the generation of $\operatorname{InsP}_{3}$ at $\mathrm{NEB}(93)$. The implication is that the fertilization calcium response in sea urchin is a one shot mechanism.

Increased sensitivity to $\mathrm{InsP}_{3}$ begins immediately after addition of the maturation-inducing hormone 1-methyladenine in starfish $(80,328)$. Absolute levels of expression of the receptor do not alter during maturation, though the spatial distribution changes (227). This increased sensitivity is due to activation of cdk1/cyclin in the GV(554), as it is attenuated in enucleated or roscovotine-treated oocytes (328). It is not due to phosphorylation of the $\mathrm{InsP}_{3}$ receptor, but is correlated with stability of the actin cytoskeleton (328). An increase in $\mathrm{InsP}_{3}$ sensitivity has also been demonstrated in frog oocytes as they mature and correlates with the formation of clusters of $\mathrm{ER}(555)$.

The evolution of the calcium signalling framework in oocytes begins even before maturation. Mammalian oocytes removed prematurely from antral follicles before ovulation have a spectrum of meiotic competence (64). Fully grown oocytes spontaneously begin maturation once removed from the ovary; growing competent oocytes will also undergo maturation on removal, though not yet full size; incompetent oocytes will not mature. The fully grown oocytes show robust repetitive calcium oscillations with a period of around 1 min; growing oocytes have oscillations, but with a period of around $5 \mathrm{~min}^{-1}$; incompetent oocytes show no spontaneous oscillations. This correlation implies a link between the appearance of calcium oscillations and cell cycle progression. The evolution of an intrinsic oscillatory mechanisms is implied, since even incompetent oocytes will respond to agonists such as carbachol and thimerosal with calcium oscillations.

There is further evolution of the calcium release mechanism as the oocyte matures. This can be uncovered by testing the system using the mammalian sperm factor (PLC $\zeta$ ). Competent but immature oocytes oscillate after injection of sperm factor, but peak attained, rate of rise and decay time of each transient are between 0.5- and 4-fold greater in mature oocytes(64). The density of $\mathrm{InsP}_{3}$ receptors increases around 2-fold during maturation and receptor clusters appear(366); increasing amounts of anti-receptor antibody are required to block the effects of $\mathrm{InsP}_{3}(473)$. In hamster oocytes it is reported that the response to InsP $\mathrm{P}_{3}$ increases gradually during oocyte maturation, and that a regenerative component of the response appears only in mature oocytes(158); releasable calcium (store filling) does not appear to vary significantly. Type $\mathrm{I} \mathrm{InsP}_{3}$ receptors are the predominant isoform in human (182) and in mouse oocytes; in mouse oocytes they increase during maturation and decrease again after fertilization(420). After fertilization in mouse oocytes, sensitivity to $\mathrm{InsP}_{3}$ decreases markedly in a use-dependent way. Down regulation is not attributable to the calcium transients themselves, but is due to $\mathrm{InsP}_{3}$ receptor occupancy $(51,240,637)$. The observation of $\mathrm{InsP}_{3}$ receptor clustering leads us to think about the structure of the endoplasmic reticulum.

\section{ENDOPLASMIC RETICULUM IN OOCYTES AND EMBRYOS}

\section{Summary}

Evidence is reviewed that points to dynamic alterations in the shape and structure of the endoplasmic reticulum during maturation and after fertilization and the link between ER dynamics and the cytoskeleton is discussed.

The endoplasmic reticulum is obviously a key element in shaping calcium signals that rely on release of calcium from internal stores. Taking frog oocytes as an exemplar, it appears 
that oocyte ER has many of the features of ER in somatic cells, for example, SERCA pumps, calsequestrin/calreticulin $(208,446)$ and store-operated calcium entry, the last downregulated by the increase in MPF as meiosis is initiated (343). The architecture of the ER can have profound effects on the spatiotemporal characteristics of calcium signals in somatic cells(20). It is striking that ER architecture evolves as oocytes mature. Interactions between ER and cytoskeleton shape calcium signals in oocytes, eggs and embryos.

Our insights into ER structure and function in the female germ line owe a lot to Terasaki's developing a relatively straightforward way of visualizing it using a lipophilic fluorescent probe $(552,556)$ and latterly with green fluorescent protein(553). Terasaki's experiments were the first to show that the lamellar-reticulate organization of the ER broke down during the peak of the fertilization calcium transient in sea urchin eggs, with the ER transiently vesiculating(552).

The same method has revealed the development or cortical ER clusters, probably stacked lamellae, in hamster, mouse and frog oocytes whose appearance correlates with maturation of the responsiveness of the calcium release mechanism. InsP $\mathrm{P}_{3}$ receptors localize to the clusters (274). The existence of these clusters may explain why injection of sperm factor (PLCS) into mature mouse oocytes can lead to repetitive calcium waves whose cortical point of origin varies from one wave to the next(64). It may also account for the enhanced cortical sensitivity of the egg cortex to sperm factor, relative to deeper cytoplasm(410). Similarly after intracytoplasmic sperm injection (ICSI) in which the sperm is slightly damaged to allow the release of its cytoplasmic contents, the origin of calcium waves is at the cortex, not at the sperm nucleus itself(472). There are no reports of ER clustering in sea urchin eggs or starfish.

During the frog fertilization wave, the clusters disappear(555), but in ascidians, worms and mammals, the clusters persist while the multiple calcium transients that characterize their oocytes continue(153, 274, 506, 528). Kline(269) has suggested that the ER fragmentation or disappearance of ER clusters in sea urchins, starfish and frog may be a mechanism that prevents the generation of repetitive transients in these species. Another correlation to note is that clusters are present in oocytes arrested with high levels of cdk1/cyclin B and MAP kinase activities. Remember that these activities decline with each repeated transient after fertilization. Carroll and his colleagues have shown that if cdk1/cyclin B activity is maintained after fertilization by the use of proteasome inhibitors or excess cyclin $\mathrm{B}$, then the clusters persist, whereas treatment of unfertilized oocytes with roscovitine, a cdk inhibitor, led to cluster disappearance(153). It seems that the clusters are dynamically maintained by high levels of cdk activity; they disappear not because calcium transients themselves effect their reorganization, but because the calcium transients cause cdk activity to fall. MAP kinase activity on the other hand does not appear to regulate ER clusters, at least in worms (530)

However, the clusters do not reappear at the next, mitotic, nuclear division when once again cdk activity is high(153). The clusters are thus peculiar to the mature oocyte, possibly because at this point in the meiotic cycle, MAP kinase activity is very high. The mos -/mouse, lacking active MAP kinase, lacks clusters (Carroll, personal communication). In mouse, what happens to the ER at first mitosis (153) mirrors what has been found universally in oocytes and embryos: that the ER surrounds first the nucleus then the mitotic spindle $(422,552)$. This gathering of the ER about the mitotic nucleus and spindle is particularly easy to see in syncytial Drosophila embryos (Figure 9), where ER becomes densely accumulated round the spindle, with highest concentrations on each side of the spindle poles, penetrating the spindle at the metaphase plate. 
It is thus the absence of spindle-associated ER in oocytes in second meiotic metaphase that is the anomaly requiring explanation. Carroll and his co-workers(153) suggest that isolating the meiotic spindle from a source of calcium that might otherwise prove a threat to a stable meiotic arrest is important, as is avoiding segregation of ER into the polar body, where it would be lost to the oocyte. Of course, lack of spindle ER does not in itself imply that cortical ER clusters should form: these are a specialization that may improve the sensitivity of the oocyte to the PLC $\zeta$ sperm factor, though it should be noted that both the sea urchin and the mos $-/$-mouse appear to fertilize quite readily in their absence.

In mouse, the first meiotic spindle shows the usual penumbra of ER (Carroll, personal communication). This suggests to me that the everyday relationship between calcium and the events of mitosis is an intimate one; only at fertilization does the ER disperse to involve the entire oocyte in a large cell cycle calcium signal; in other circumstances, the relationship is much more discreet.

The disposition of ER within the cell is known to depend on interactions with microtubules and their motors(15). Presumably the centripetal accumulation around the spindle is due to dynein-based interactions, while the centrifugal clustering of the fertilizable oocyte may depend on kinesin-based interactions with astral microtubules. A centripetal microtubuleassociated accumulation of ER is also seen in sea urchin zygotes around the pronuclei as they prepare to fuse (our unpublished observations), another germ-line event that is controlled by a calcium signal $(173,485)$. Cyclin/cdk phosphorylation of the actin cytoskeleton is also involved in modulating ER sensitivity to $\operatorname{InsP}_{3}(328)$. One of the most beautiful examples of microtubule/ER interactions is seen in ascidian embryos after fertilization.

The fertilization calcium waves in ascidian embryos, as is common, initiates at the point of sperm egg fusion, usually within the animal hemisphere(447). Thereafter, the site of initiation migrates with the sperm nucleus and aster around the cortex towards the vegetal pole $(358,359,447)$. The sperm aster in association with actin-based cortical contractions driven by the calcium waves themselves reorganizes and segregates the oocyte cytoplasm to generate a nipple of ER-containing cytoplasm at or near the vegetal pole $(127,130,358$, 506). The accumulation of ER is then the locus of origin of the second set of calcium waves that occur as the oocyte enters the second meiotic division and which originate at this ERenriched pacemaker. The ER is separated, incidentally, by a cordon sanitaire of mitochondria from the rest of the cytoplasm, an arrangement analogous to the disposition of pacemaker ER at the pole of pancreatic acinar cells(20). The ER accumulation itself, though, does not generate the pacemaker activity: the activity is closely associated with the sperm aster and there is evidence that it may be due to localization of the egg activating factor introduced by the ascidian sperm(65).

The gentle gavotte orchestrated by the post fertilization calcium waves in ascidian oocytes may be responsible for setting up the dorso-ventral axis of ascidian embryos. This brings us to our final topic: the contribution of calcium signals to embryonic pattern formation.

\section{CALCIUM, AXES AND PATTERN FORMATION IN EMBRYOS}

\section{Summary}

Evidence that calcium signalling plays a key part in the development of patterning in early embryos is reviewed. Morphogenesis in ascidian, frog and zebrafish embryos is briefly set out to provide a description of the developmental context in which calcium signals act. Intracellular calcium waves that may play a role in axis formation in ascidian 
are discussed. Evidence that the Wingless/calcium signalling pathway is a strong ventralizing signal in Xenopus, mediated by phoshoinositide signalling, is reviewed. The central role that calcium channels play in morphogenetic movements during gastrulation and in ectodermal and mesodermal gene expression during late gastrulation is demonstrated. A hypothesis that reconciles conflicting data in the field is suggested. There is discussion of how the optical transparency of zebrafish oocytes has permitted confirmation of the experiments in Xenopus embryos, themselves opaque and largely unsuited to calcium imaging. Experiments in zebrafish provide a strong indication that calcium signals are essential for pattern formation and organogenesis.

Many more genes are involved during embryonic development of higher organisms than are expressed during juvenile and adult life (519). Development is a process of huge complexity even in simple metazoans like $C$. elegans. Understanding the part that calcium signals play in embryogenesis will be difficult because of the complexity, not of the calcium signals themselves, but of the developmental context in which they act. Nonetheless, there are simple macroscopic principles to be drawn from observing development; experiments have already begun to demonstrate the importance of calcium signalling in this macroscopic context.

The fundamental macroscopic principle in embryology is that of the embryonic axes: anterior-posterior, dorso-ventral and left-right. In some embryos, all the axes are set up within the oocyte in the ovary, so that the symmetry of the embryo may be predicted by inspection of the oocyte. In other embryos, one or more axes crystallizes during early development due to a symmetry breaking event, for example sperm entry, and cannot be predicted in advance. Another macroscopic principle of development is that of coordinated cell migrations at a scale comparable to the size of the embryo. Examples include epiboly is fish embryos, gastrulation (formation of the gut) and neurulation (formation of the spinal cord). A third principle is organogenesis, where once the overall body plan is laid out, local differentiation gives rise to organs such as eye, kidney and liver. There is evidence that calcium signals are important in both cell migration and axis formation and some suggestion that they may also be involved in organogenesis (595).

The majority of the data linking calcium signals and pattern formation comes from ascidian, frog and zebrafish embryos. While sea urchin embryos have contributed enormously to our understanding of gene regulatory networks and cell specification in early embryos (112), it has been shown only that lithium and protein kinase $\mathrm{C}$ alter vegetal cell fate by altering the expression of forkhead transcription factors $(332,333,339)$. These observations may be linked to the interesting finding that the micromeres (the most vegetal cell type) exhibit marked, oscillating, InsP $\mathrm{P}_{3}$-driven calcium transients at the time that their fate is determined $(629,630)$.

\section{A. Calcium and dorso-ventral axis formation}

Dorso-ventral axis specification occurs early during development in ascidian and frog embryos, at the one cell stage; in fish, axis specification occurs later. All involve calcium and phosphoinositide signalling.

1. Ascidian embryos-The migration of sperm and aster towards the vegetal pole of the ascidian oocyte during the first phase of calcium spikes that precedes first polar body extrusion (Figure 10) is accompanied by a profound cytoplasmic reorganization involving marked cortical contractions in which ER is transported to a nipple-shaped extrusion in the vegetal hemisphere known as the contraction pole $(130,358,502,505,506)$. In the same reorganization, mitochondria are concentrated towards the equator on one side of the 
embryo. The mitochondrial region is known as myoplasm, as it contains both the mitochondria and the cytoplasmic determinants that will specify and fuel the muscular tail of the swimming embryo (239). Since the cortical, actin-based contractions are dependent on the calcium spikes and the ooplasmic segregation dependent on the contractions $(239,447$, 506), it is firmly established that the calcium waves are essential for embryonic pattern formation.

The second set of calcium spikes originate from the contraction pole and their direction of propagation defines the dorso-ventral axis, in the sense that it is a marker of the axis (Figure 10). It is reasonable to suppose that this second spike train consolidates the axis in the embryo; however, the subcortical rotation that establishes the axis occurs in first mitotic interphase, once the second set of transients have ceased. The accumulation of ER and the putative ascidian sperm factor in the contraction pole pacemaker offers a possible explanation of why it is the point of origin of the calcium waves (65), by analogy with acinar cells(20), though in fact the animal pole is more sensitive to $\operatorname{InsP}_{3}(130)$. This latter finding in fact implies that the contraction pole harbours a Ins $\mathrm{P}_{3}$ generator, for example, the sperm factor. By elimination, it has been shown that neither accumulation of PtdInsP $\mathrm{P}_{2}$, nor local calcium influx nor local accumulation of endoplasmic reticulum can explain the origin of the calcium waves, leaving localization of the sperm factor as the only explanation (65). There are no data to demonstrate that the contraction pole calcium waves are essential for maintenance of the dorso-ventral axis. However, the observation that it is possible to generate animal pole waves in place of the usual contraction pole waves suggests a way of testing the idea(130).

Mouse eggs show a similar migration of repetitive fertilization calcium transients from the site of sperm entry to the vegetal pole $(116,127)$. Whether they perform a similar function to those in ascidian is unclear. However, it has been found that the site of sperm entry specifies the embryonic-abembryonic axis in mouse embryos to within $30^{\circ}(432,433,636)$, so it is conceivable that these transients specify an axis in mouse embryos; against this idea is the finding that axis specification is correlated with the disposition of the male and female pronuclei, rather than sperm entry itself (211).

2. Frog embryos-One celled frog embryos also undergo a cytoplasmic reorganization driven by sperm entry in the animal pole. Cortical vegetal cytoplasm slides upwards towards the equator to define the dorsal region of the embryo in part through specifying a dorsal or ventral fate for overlying mesoderm. The rotation aligns cortical microtubules, allowing asymmetric transport of determinants towards the equator on the dorsal side $(201,371)$. It is not known whether calcium signals accompany this movement. Three broad phases of signaling have been identified during dorso-ventral specification in frog embryos $(201,497)$ (Figure 11). The first is during the mid-blastula stage when vegetal cells convert cells at the animal/vegetal boundary to a mesodermal fate. This phase of signaling involves fibroblast growth factor (FGF) and activin (transforming growth factor $\beta$-family) pathways, but there is no evidence on balance that it is more than permissive for dorso-ventral axis formation. The second phase involves the vegetally-located dorsalizing Nieuwkoop centre whose role is to cause the appearance of the Spemann Organizer that carries out induction of dorsal tissues in the early gastrula, for example neural induction. Signals in the second phase (that of dorsoventral axis specification) are carried by Bone Morphogenetic Protein and Wingless (Wnt) signaling and in the third phase by proteins such as Noggin, Follistatin, Xnr3, Cerberus, Frzb and eFGF (Fibroblast Growth Factor) generated by the Spemann organizer.

i. Fibroblast Growth Factor Signals and phospholipase $\mathbf{C} \boldsymbol{\gamma}$ : It is known that the FGF signaling pathway is involved in mesoderm induction but not in dorso-ventral patterning (201). FGFR1 is the receptor subtype that effects induction of mesoderm and its main 
effector system is the Ras/MAP kinase cascade (574). It has however been found that phosphorylated (activated) PLC $\gamma 1$ associates with active FGFR1 in presumptive mesoderm cells (454) along with Sos, an upstream regulator of Ras. The obvious implication is that calcium signals may play a role in mesoderm induction and indeed, exogenous FGF1R can activate $\mathrm{PLC} \gamma$, production of $\mathrm{InsP}_{3}$ and calcium release (394) in embryos. This conjecture seems unlikely to obtain, as a mutant Y766F FGF1 receptor was unable to activate the phosphonositide pathway, while still being quite capable of inducing mesoderm (394). Taking as a starting point the very substantial localization of $\operatorname{InsP}_{3} \mathrm{R}$ to the animal hemisphere in Xenopus embryos (290), and the known involvement of the MAP kinase signaling pathway, a systems biology approach has replicated periodic calcium signals driven by FGF (119) and has suggested that the calcium oscillations are driven by a pattern generator in the prospective dorsal ectoderm. The model implies that the calcium signals are central to mesoderm induction. Though the model is contradicted by the available evidence, it is the only study that gets to grips with a quantitative analysis of cellular calcium signaling during development and is recommended on these grounds alone.

\section{ii. The Nieuwkoop centre: the Wingless (Wnt) pathway and calcium signaling: Early} embryologists knew lithium to be a dorsalizing agent; we know lithium to be a phosphoinositide signalling antagonist(39). The dorsalizing effects of lithium could be rescued by myo-inositol (a signature of the phosphoinositide pathway) or by a protein kinase $\mathrm{C}$ agonist(54). Ectopic stimulation of the pathway using an exogenous G-protein-linked 5-

HT receptor and treatment with 5-HT led to ventralization when agonist was applied (21). It was also found that lithium-sensitive inositol phosphate production occurred during mesoderm induction (349). Treating embryos with an anti- $\mathrm{InsP}_{3}$ receptor antibody dorsalized them (289). These observations strongly suggest that calcium signalling from intracellular stores driven by $\mathrm{InsP}_{3}$ defines an active ventralizing mechanism in Xenopus.

However, as with many other developmental pathways that control cell fate (409), the dorsoventral system in the frog is a yin-yang mechanism in which a dorsalizing pathway competes with a ventralizing pathway. Both pathways are based on signalling through the Wnt/Frizzled agonist/membrane receptor family. The canonical Wnt pathway, so called because it was the prevalent Wnt (Wingless) pathway identified in Drosophila does not involved calcium at all. Agonists of this pathway such as Wnt-8 act through Frizzed via an unknown mechanism that may involve casein kinases to activate Dishevelled; in turn, Dishevelled inhibits glycogen synthase kinase (GSK), leading to downregulation of phosphorylation of its substrate $\beta$-catenin (a protein associated with cadherin in cell-cell adherens junctions, but also a nuclear transcription factor); phosphorylation of $\beta$-catenin leads to its proteolysis, so by inhibiting GSK, Dishevelled stabilizes $\beta$-catenin. Dishevelled translocates dorsally as a consequence of cortical rotation (371). This pathway is dorsalizing and it is dominant, in that ectopic ventral expression of Wnts in Xenopus embryos leads to dorsalization, in the shape of a secondary body axis $(388,500)$. mRNA of Wnts 1 and 8 mimic the Nieuwkoop centre when injected into frog embryos $(498,500)$. These Wnts signal via the subclass of Frizzled receptors represented by Rfz1 (628).

If the canonical Wnt pathway is the yang then the yin is the $\mathrm{Wnt} / \mathrm{Ca}^{2+}$ pathway. A second class of Wnts do not themselves induce an obvious phenotype when overexpressed, but do antagonize the effects of ventral overexpression of the canonical Wnts (567) and themselves signal to maintain ventral cell fate $(225,286,289)$. They signal via pertussis toxin-sensitive heterotrimeric G-proteins (495). These weaker Wnts activate protein kinase C $(370,415)$, in an echo of the rescue of lithium inhibition by a protein kinase $\mathrm{C}$ agonist mentioned above(54); the weaker Wnts presumably signal through protein kinase $C \beta$, as overexpression of protein kinase $\mathrm{Ca}$ induces dorsal competence in ventral tissue (413). They interact with a subset of Frizzled receptors exclusive to the Wnt/Ca pathway and distinct 
from the subset that mediate canonical Wnt signalling: a panel of exogenous mouse Frizzled receptors either induced PKC activation (ventralizing) or both siamois and Xnr-3 expression (dorsalizing products downstream of $\beta$-catenin) in Xenopus; no receptor activated both pathways (483). The weaker Wnts (here Xwnts 5A and 11) and their Frizzled partners activate CaM Kinase II in Xenopus embryos; CaM kinase II activity is higher ventrally than dorsally, and suppressed by ectopic expression of a dominant-negative construct of Xwnt-11 (286). Microinjection of constitutively active CaM Kinase II ventralizes embryos, while a dominant negative CaM Kinase II construct induces dorsal structures (286); the $\operatorname{InsP}_{3}$ / calcium/CaM Kinase II pathway controls the expression of Xvent1, a ventral specification gene $(286,289)$.

A more precise identification of the identity of the trimeric GTP binding proteins involved in dorso-ventral specification has been attempted using anti-peptide antibodies directed at the C-terminal of three difference G-protein a subunits. Injection of anti-Gas/olf and anti$\mathrm{Gai} / \mathrm{o} / \mathrm{t} / \mathrm{z}$ antibodies into Xenopus oocytes led to dorsalization, the former antibody showing the much stronger phenotype (288), implying that the Gas G-protein family is responsible. Anti-Gas/olf antibody induced the organizer genes chordin and noggin in ventral explants and produced a strong second axis with well-developed anterior structure, while the anti$\mathrm{Gai} / \mathrm{o} / \mathrm{t} / \mathrm{z}$ antibody induced chordin strongly, but noggin only weakly, with anteriormost structures absent in the second axis. These phenotypes are very similar to that seen after injection of $\mathrm{InsP}_{3}$ receptor antibody $(289,290)$. An anti-Gaq11 antibody was without effect, though it was shown that the subunit was present and the antibody functionally-inhibitory. It was not possible to demonstrate any effect of the adenylyl cyclase inhibitors SQ22536 and MDL12330A on embryonic development, despite the demonstration that both inhibitors markedly reduced adenylyl cyclase activity in animal caps (dissected ectoderm). Signalling through $\beta \gamma$ subnits was abrogated by expression of $C$-terminus of the $\beta \gamma$ binding protein $\beta$ ARK1 (an Adrenergic Receptor Kinase) and a non-functional $\beta$ ARK1 control: this led to formation of an ectopic axis (dorsalization). Finally, it was shown that $\beta 2$ adrenergic receptor/transducin $\beta \gamma$ dimer-expressing animal caps generated calcium transients in response to arterenol (implying a phosphoinositide response) and that this response could be prevented by microinjection of the anti-Gas/olf antibody; a similar result was obtained using exogenous muscarinic acetylcholine receptor 1 and the anti-Gaq11 antibody.

These data are unexpected. The usual route from receptor to PLC $\beta$ activation and calcium release is through Gaq. Gai/o can stimulate PLC $\beta$ by dissociation of the $\beta \gamma$ dimer. Gas subunits are most commonly linked to activation of adenylyl cyclase, but no effects of adenylyl cyclase inhibition were found. While it was clearly shown that stimulation of Gas could generate calcium signals in animal caps, these signals were localized to a small subset of animal cap cells, whereas stimulation routed through Gaq led to widespread calcium release (288). More fundamentally, it is clear that weaker Wnts signal through pertussis toxin-sensitive G-proteins (Gao/i) (495). There is thus no place for pertussis-insensitive Gas in the Wnt/Ca pathway. Though the authors do not discuss this point, they do suggest that the weaker phenotype seen with the anti-Gai/o/t/z antibody may be due to a lower capacity for functional inhibition. These data are therefore weakly consistent with a role for Gai/o in the Wnt/Ca signaling pathway.

Whatever the role of Gaq in dorso-ventral axis formation, it has been used to adduce further evidence for $\mathrm{InsP}_{3}$ signalling in dorsoventral specification. A constitutively active Gaq subunit contruct induced dorsalization when expressed ventrally, but not dorsally, in Xenopus blastulae (291). Given that its companion paper (288) shows that blocking Gaq has no effect, this is paradoxical. The resolution of the paradox is that constitutive activation was shown to lead to desensitization of the $\mathrm{InsP}_{3}$ receptor in the embryos (291). The authors attribute this desensitization to use-dependent inhibition (621). The observation 
is further evidence that maintaining ventral structures requires a patent phosphoinositide pathway.

It has also been shown using Xenopus animal caps that the path from the weaker Wnts to calcium runs through Dishevelled (Dsh). Animal caps are convenient pieces of embryo that can be induced to produce dorsally-competent cells by the Nieuwkoop centre, but can also take on ventral fates. Dsh appears to be a multivalent protein that interacts with the GSK/ $\beta$ catenin pathway via a so-called DIX domain. A Dsh $\triangle$ DIX construct activates both PKC and CaMKII in animal caps (484). The effect is not blocked by pertussis toxin, indicating that Dsh is downstream of the Frizzled receptor-G-protein component of the pathway. A morpholino oligonucleotide directed against endogenous Xdsh blocks activation of PKC measured enzymatically; as a control, it was shown that the morpholino construct did not block the effect of a truncated but effective exogenous Xdsh mRNA with which the morpholino could not hybridize. This is a key observation, as it is one of only two sets of data in Xenopus that target endogenous Wnt/Ca signaling rather than use exogenous Wnt or Frizzled constructs; the other data set is that showing differential dorso-ventral activation of CaMKII (286).

The Dsh $\Delta$ DIX construct has been shown to stimulate the so-called Planar Cell Polarity (PCP) pathway during gastrulation in Xenopus, a point to which we shall return in due course; however, it also rescues the aberrant phenotype of a Wnt 11 loss of function during gastrulation (545). By using a deletion construct (Dsh $\triangle$ DEP $\triangle$ PDZ) lacking the DEP and PDZ domains that are known to be required for activation of the PCP pathway by Dsh, it was demonstrated using exogenous Frizzled (Xfz-7) expression that stimulation of PKC in animal caps was independent of the PCP pathway; nonetheless, a DEP domain construct did reduce membrane localization of ectopic $\mathrm{PKC}$, implying that this domain plays some part in Dsh stimulation of the Wnt/Ca pathway (484).

The calcineurin-NFAT pathway has been found to be involved in dorso-ventral specification and may thus lie downstream of the calcium signal. A constutitively active, calcineurinindependent NF-AT ventralizes embryos (457), inhibiting activin-induced morphogenesis in an echo of the consequences of Wnt-5A overexpression (387). It also prevents the dorsalization induced by ectopic Xwnt-8. A dominant-negative NF-AT stabilized coexpressed $\beta$-catenin, the NF-AT antagonism of the dominant Wnt pathway acting downstream of disheveled (457). Measurement of the evolution of calcineurin activity by assay of phospho-NF-AT implied that calcineurin was activated transiently at the start of mesoderm induction and the transient dephosphorylation of NF-AT was prevented by microinjection of BAPTA. A potentially conflicting, dorsalizing activity of calcineurin may be to inactivate TGF $\beta$ family signaling via the immunophilin FKBP12 (591). Ventral expression of a Xenopus FKBP homologue resulted in axis duplication of muscle and pronephros (400). A mutant able to bind to TGF $\beta$ but unable to bind to calcineurin was ineffective. Microinjection of a constitutively active calcineurin catalytic subunit was also capable of inducing a secondary axis. Simultaneous overexpression of XFKBP and either XBMP 4 or its downstream effector Xmad 1 did not lead to duplication, implying that calcineurin is antagonizing BMP 4 signalling, possibly by inhibiting the BMP 4 receptor. In situ hybridization demonstrated that XFKBP was localized dorsally and anteriorly in neural structures after gastrulation. XFKBP was detected on Western blots in the unfertilized eggs and increased gradually after the transcription of the zygotic genome at mid-blastula stage (400). However, it was undetectable by in situ hybridization until after gastrulation. Nonetheless, its anterior-dorsal localization in later embryos suggests very simply that calcineurin is on a dorsalizing pathway in dorsal mesoderm and on the Wnt/Ca ventralizing pathway in ventral mesoderm and that this can be explained by the presence of XFKBP dorsally and its absence ventrally. This conjecture is supported by the observation that three 
inhibitors of calcineurin that require FKBP for their action are effective in affecting dorsal structures in embryos when injected dorsally (preventing the late stage development of heart, liver, gut and somites) but have no effect on development when injected ventrally (633). This argument implies a dorsal calcium signaling system distinct from the ventral Wnt/Ca pathway. We return to this in sections VA2iv and $v i$.

The calcineurin-NFAT pathway provides the clearest exposition in Xenopus of the general observation that a ventralizing $\mathrm{Wnt} / \mathrm{Ca}^{2+}$ pathway antagonizes a dorsalizing canonical Wnt pathway during dorso-ventral axis formation in frog embryos. It is ironic, though, that the teratogenic effects of lithium that first led to the hypothesis that the phosphoinositide signalling pathway was involved in pattern formation (39) can only be interpreted ambiguously in Xenopus. It has been reported that L 690330, a drug that blocks inositol monophosphatase and so depletes the polyphosphoinositide pool preventing phosphoinositide signaling, has no effect on dorso-ventral polarity in Xenopus (268); the result very evidently flies in the face of all the other evidence presented in this section that points to a role for calcium as a ventral specification signal as well as the evidence that L690330 dorsalizes zebrafish embryos, inducing duplicate heads (600). Until this study was published, the dorsalizing effects of lithium had been attributed to its action as a noncompetitive inhibitor of inositol monophosphatase (39); in sea urchin embryos axis disruption caused by lithium is accompanied by the expected decrease in $\operatorname{InsP}_{3}(92)$. An alternative molecular target was proposed: lithium inhibits the activity of GSK3 $\beta$ both in vivo and in vitro $(203,268)$ : its dorsalizing effects can happily be ascribed on this basis as inhibition of GSK, a component of the canonical dorsalizing strong Wnt pathway, would stabilize dorsal-specifying $\beta$-catenin. However, it has not been shown directly that myoinositol reverses lithium inhibition of kinase activity, only that it can reverse the effects on downstream targets such as $\beta$-catenin (203). Its effects on GSK's downstream targets in oocytes can be reversed by adding myo-inositol, as can the effects of a dominant negative GSK (203), results inexplicable in other terms than stimulation of the phosphoinositide pathway (457).

\section{iii. Gastrulation and convergent extension of the notochord, somites and neural plate:} Large scale cell movements at gastrulation determine the final body plan. The two major cell movements at gastrulation are involution, which refers to the migration of cells through the blastopore that converts a single- into a two-layered embryo and convergent extension, a movement that gathers cells towards the midline dorsally (convergence) and elongates the dorsal midline by an anteriorward elongation through cell intercalation (extension). Convergent extension movements set up the anterior-posterior organization of the vertebrate, defining the head-to-tail pattern of the embryo $(215,257,258,399)$. The weaker Wnts first hit the headlines as signaling molecules that antagonized convergent extension, some time before their importance in ventral specification became known $(123,389)$. In fact, their effects on convergence extension are the other side of the coin of ventral specification. The ability to undergo convergent extension is a property of dorsal margin cells: ventral margin cells lack this propensity, but can acquire it when treated with dorsalizing molecules. The yin-yang balance of Wnt signaling is reflected in the specification of the propensity for convergent extension, just as it is in the specification of dorso-ventral gene expression: the canonical pathway promotes convergent extension, the Wnt/Ca pathway prevents it, almost certainly by suppressing transcription of Xnr-3, a factor essential for the emergence of convergent extension behaviour $(285,499)$. Data from the analysis of convergent extension offer further evidence of the importance of calcium signaling in dorso-ventral specification.

The early specification of convergent extension can be studied in animal caps treated with activin (to induce mesoderm). It has been shown that the Wnt/Ca pathway antagonizes the 
canonical pathway both by PKC-mediated phosphorylation of Dsh upstream of GSK $\beta$ and by downstream CaM Kinase II mediated phosphorylation of a transcription factor complex (285), explaining the suppression of Xnr-3 ventrally, where CaM Kinase II activity has been shown to be high (286).

The cell movements that comprise convergent extension can also be studied in activintreated animal caps. By the time of cell movement, the Wnt signaling landscape has changed and canonical Wnt signaling is no longer required $(545,588)$. Instead, the planar cell polarity pathway that signals via JNK is invoked. This second, non-canonical Wnt pathway also signals through Dsh to activate JNK. It was shown using truncated Dsh constructs that were unable to activate the canonical Wnt pathway that the canonical pathway was not necessary for convergent extension. Using the Dsh $\triangle \mathrm{DEP}$ and Dsh $\triangle \mathrm{PDZ}$ constructs described earlier that block the PCP pathway in Drosophila, the PCP pathway was shown to be essential (588). Activation of the PCP pathway is associated with polarized cell motility and filopodial extension (588). So while the use of Dsh constructs demonstrated the importance of the Wnt/Ca pathway in early dorsoventral specification and the absence of PCP signalling, the same approach shows the reverse during convergent extension. The necessity of PCP signaling in the parallel and simultaneous convergent extension movements in neural ectoderm has also been demonstrated (587). The PCP pathway may also be capable of interacting with the Wnt/Ca pathway, as convergent extension is specified (84). However, this interaction is very hard to interpret, as both under- and over activation of the PCP pathway can lead to abnormal cell adhesion $(84,545)$, making the usual logical inferences from overexpression and dominant negative approaches impossible to draw.

With Yang out of the picture in regulating the cell movements of convergent extension, is there any need for Yin? Possibly. During involution, the mesodermal cells remain separated from the overlying ectoderm. This appears to involve the regulation of cell-cell adhesion by the Wnt/Ca pathway (618). The development of the cleft (Brachet's cleft) that appears between mesoderm and ectoderm during gastrulation was prevented by injection of an antisense morpholino to the frizzled receptor Fz-7 at the 4-cell stage. The defect could not be rescued by dominant negative GSK3, nor stabilized $\beta$-catenin nor activated transcription factor TCF3, ruling out the canonical Wnt pathway. Nor was it rescued by Dsh $\triangle \mathrm{DIX}$, nor activated Cdc42 (a small G-protein component of the PCP pathway), ruling out PCP signaling. To reinforce this conclusion, dominant negative Wnt-11 was ineffective, though it blocked PCP-mediated convergent extension as expected. The evidence that the $\mathrm{Wnt} / \mathrm{Ca}$ pathway is mediating the formation of Brachet's cleft is as follows: cleft formation is blocked in embryos injected with pertussis toxin and this could be rescued by injection of $\mathrm{PKCa}$, but not by coexpression of Fz-7; PKCa rescues the defect caused by the Fz-7 morpholino; phosphorylation of PKC in dorsal cells was prevented by the Fz-7 morpholino. Using a neat assay in which FGF-induced (mesodermally-directed) animal cap cells were placed in a ball on untreated blastocoel roof explants (BCR: the underside of the animal cap), it was found that expression of Fz-7 led to the continued separation of the two explants. In the absence of Fz-7, the animal cap cells sank into the BCR. The separation induced by $\mathrm{Fz}-7$ was prevented by pertussis toxin and toxin inhibition in turn rescued by PKCa.

Mesoderm-ectoderm adhesion is clearly controlled by the Wnt/Ca pathway, not the PCP pathway. Again, the simplest way to view these results is to infer that dorsal mesodermectoderm adhesion is another facet of the dorsoventral specification process in the early blastula. But in this case, the Wnt/Ca pathway is acting dorsally to specify cell adhesiveness, not directly antagonizing the dorsalizing canonical Wnt pathway. These experiments do give rise to a contradiction. The authors conclude, based on the observation that Dsh $\Delta$ DIX does not rescue the Fz-7 morpholino, that disheveled plays no part in the Wnt/Ca pathway. As we 
have seen, a later study showed that a morpholino directed against Dsh blocks activation of PKC (484) and that contrary to the expectation raised by the Brachet's cleft experiments, Dsh $\triangle$ DIX can activate both PKC and CaM Kinase II in animal caps. Sheldahl and colleagues (483) suggest that there may be an overlap between the Wnt/Ca and PCP pathways in that the PCP pathway may stimulate PKC, but they do not comment on the conflicting results obtained with Dsh $\triangle \mathrm{DIX}$.

Another hint that the Wnt/Ca pathway may regulate cell movement comes from calcium measurements in explants: there are striking calcium signals associated with convergent extension in Keller explants of the dorsal blastopore lip at gastrulation; their observable occurrence is reduced from $80 \%$ to $40 \%$ by suppressing all Wnt signaling with an Nterminal fragment of the Xenopus Frizzled-8 receptor (586).

An attentive and by now perhaps frustrated reader of this section will have noted that the preceding paragraph makes the first mention of the measurement of calcium signals themselves. There are good reasons for the lack of data. Xenopus embryos are large and lend themselves readily to microinjection. The well-worn experiment in Xenopus development is to microinject mRNA or protein, observe the perturbed developmental phenotype and infer the involvement of the relevant regulatory pathway. But Xenopus embryos are opaque and so do not at all lend themselves to the optical methods employed by cell biologists and cell physiologists. The salient feature of the demonstration of calcium signals during convergent extension in Xenopus is the use of relatively thin sheets of excised tissue together with multiphoton microscopy to image calcium levels. The method allows good imaging at depth in highly scattering and absorbing tissues, with proportionately less photobleaching and tissue damage (616).

In so-called Keller explants of the blastopore lip customarily used to study convergent extension movements, large, multicellular calcium waves were observed that propagated 15-20 cell diameters $(300-400 \mu \mathrm{m})$ at a rate of around $5 \mu \mathrm{m} . \mathrm{s}^{-1}$, or more frequently 5-10 cell diameters at a rate of 2-3 $\mu \mathrm{m} . \mathrm{s}^{-1}$. The waves originated in 2-4 adjacent cells, apparently randomly in space and time; after propagation, intracellular calcium remained elevated for 2-4 minutes before subsiding uniformly. The waves varied in amplitude (calcium concentration was not determined) and timing, but occurred with a mean frequency of $0.7 / \mathrm{hr}$ (range $0.3-3 / \mathrm{hr}$ ). The calcium wave accompanied a wave of contraction. Calcium flashes in 2-4 cells were also observed with a similar rise time but shorter duration. These may represent abortive waves. Waves were not observed in ventral mesoderm or animal caps isolated from the same embryonic stage (stage 10). When inspected, similar waves were observed in the neural ectoderm present in the Keller explant; these were not quantified. The waves were absent in explants treated with either thapsigargin or BHQ, treatments that would be expected to deplete intracellular calcium stores. Significantly, these agents prevented convergent extension, but not the expression of dorsal mesoderm genes (Xnot, chordin) and ventrolateral markers (MyoD, Xwnt-8). Ventral (Xvex), endodermal (Edd), and ectodermal (NCAM) markers were not expressed, demonstrating that there had been no alteration in tissue specification. These data indicate that calcium signals are essential for proper convergent extension during gastrulation, possibly through the regulation of cell motility and migration (585). The authors point out that such intercellular calcium waves may provide a means of coordinating the large numbers of cells involved in gastrulation movements in vertebrates. The mechanisms of origin of the waves are unknown. At this point and position in development, calcium waves do not alter developmental expression of key embryonic dorsomesodermal specification genes.

iv. Induction of neural tissue and somites: Involution and convergent extension during gastrulation shape the embryo and generate a raised ridge of cells in the surface ectoderm 
running meridionally from the blastopore towards the animal pole (201). The ridge of cells extends during gastrulation: its fate farthest from the blastopore is ultimately to form anterior structures (encephalon, eyes and so on). Those cells closest to the blastopore go on to form posterior structures (spinal cord). Involuting cells also align beneath the ectodermal ridge and differentiate along the anterior posterior axis, their mesodermal origin determining that they ultimately form muscle and bone. Cell fate is determined by signals from the Spemann Organizer as gastrulation proceeds. Noggin/chordin signaling, for example, induces neural tissue when acting on ectoderm and muscle and bone (from somites) when acting on mesoderm. An accepted view is that these and other molecules from the Spemann organizer do not themselves have dedicated downstream signaling pathways, but instead bind and inactivate other signals (BMP, Wnt) that would otherwise induce more ventral cell fates. Thus the natural propensity of ectoderm is to form neural tissue and only the local presence of BMP signals produces an epidermal fate; latero-ventral mesoderm constitutively forms somites $(201,466)$. Antero-posterior pattern formation is thought to be determined by a second process, caudalization, that switches cell fates to form more posterior structures.

The first demonstration of an ionic signal during neural induction was the observation that a very small 0.1 unit increase in $\mathrm{pH}$ was essential for the expression of a neural engrailed homologue, en-2 (468). The increase was measured in dorsal ectoderm in Keller explants like those used to detect the calcium signals during convergent extension discussed in the previous section. It could be abolished by a choride-bicarbonate exchange antagonist (H2DIDS) or by manipulating extracellular sodium and choride concentrations. Expression of a notochord (mesodermal) marker was not affected by preventing the $\mathrm{pH}$ increase. However, H2DIDS did prevent convergent extension of the mesoderm. Control ventral ectomesodermal explants showed no change in $\mathrm{pH}$. Interestingly, dorsal tissue deprived of the planar inducing signal from the mesoderm by excision of the mesoderm from the explant showed a $0.1 \mathrm{pH}$ unit alkalinization, perhaps implying that a fall in $\mathrm{pH}$ was associated with an epidermal cell fate.

There is evidence that calcium signals may play a role in neural induction. A key observation is that the neuralizing signal noggin triggers a calcium transient in animal cap cells of the salamander Pleurodeles waltl (302) and induces the immediate early gene fos via CREB, a calmodulin-responsive transcription factor (304). The calcium signal and fos induction can also be generated by treatment with an L-type calcium channel agonist, BayK 8644; the effects of either stimulus are blocked by an L-type channel antagonist (304). Moreau's group had earlier shown in the salamander that animal cap explants would develop over 5-6 days in culture into neurons and glia when treated with concanavalin A (a lectin that induced a calcium signal in these cells) or with an L-type channel agonist (391). The effect was prevented by L-type channel antagonists and by BAPTA. Notably, BAPTA treatment led to an epidermal cell fate, confirming that BAPTA was not simply causing cell morbidity. Similar calcium signals and neural cell faste were seen in response to phorbol ester stimulation of (presumably) protein kinase $\mathrm{C}$, and in response to caffeine and ryanodine (391). It had previously been demonstrated that protein kinase $C \beta$ induced neural cell fates (413) and that PKC signaling of neuralization was enhanced by the cAMP pathway (414).

These data point to the importance of voltage-sensitive calcium channels in neural specification, findings underlined by the observation that the distribution of the L-type channels determined in salamander embryos using a fluorescent dihydropyridine antagonist (STbodipy-DHP) evolved in dorsal ectoderm in line with the ectoderm's competence to be induced as neural tissue (303). A similar approach in Xenopus found the dihydropyridinebinding channels to be localized to the marginal zone dorsally, but not ventrally (419) and weakly in ectoderm. Antibody directed against the L-type calcium channel $a_{1}$ subunit 
revealed that expression of the subunit was detectable at blastula stage in Xenopus, where its localization was cytoplasmic; at gastrulation it was found in the plasma membrane of dorsal and ventral ectoderm, with some labeling in mesoderm, but none in endoderm (122). mRNA encoding the major ion-conducting subunit $\left(a_{1} S\right)$ of the L-type channel was found in embryos from stage 7 (mid-blastula) at the time that zygotic transcription begins (419). It was expressed at later stages in presomitic mesoderm and in somites. Note that the localization as determined by in situ hybridization suggests a predominantly mesodermal localization, but that as determined by antibody, a predominantly ectodermal localization.

Overexpression of $\mathrm{a}_{1} \mathrm{~S}$ increased intracellular calcium in dorsal mesoderm and led to axis duplication (dorsalization). Overexpression of other L-type calcium channel subunits $\left(\beta_{1 b}+a_{2} \beta_{2}+/-a_{1}\right)$ also caused axis duplication. The misexpression of the dorsal signal chordin due to overexpression of $\mathrm{a}_{1} \mathrm{~S}$ could be antagonized by co-injection of BMP 4 mRNA, an echo of the finding already mentioned that dorsalization by overexpression of FKBP in the calcineurin pathway was also rescued by BMP 4 (400). Increased $a_{1} S$ led to strong ventral expansion (dorsalization) of dorsal mesoderm genes (Cereberus, chordin) and inhibition of the expression of ventral mesoderm genes (Vent-1, Wnt-8). Ventral marginal zone explants from $\mathrm{a}_{1} \mathrm{~S}$-injected embryos also strongly expressed two dorsal mesoderm markers (Pintallavis, MyoD) and appeared to undergo convergent extension. The key experiment in this study however, is the use of specific channel toxins (calcicludin or taicatoxin) to determine the role of L-type calcium channels in embryos in the absence of exogenous constructs. Gastrulation was prevented. Dorsal marginal zone explants treated with the toxins failed to express MyoD or to converge and extend (419). The use of these toxins thus indicate that L-type calcium channels are essential for correct specification of dorsal mesoderm, as embodied in both cell movements and dorsal gene expression.

Our discussion of L-type calcium channels started out with neural induction in ectoderm in the salamander Pleurodeles waltl and ended up back before we started, before convergent extension, with a clear role for L-type calcium channels in dorsal mesoderm specification in Xenopus. Indeed, expression of the $a_{1} \mathrm{~S}$ subunit was found to be weak in ectoderm and overexpression of $\mathrm{a}_{1} \mathrm{~S}$ subunit in Xenopus did not lead to any detectable expression of a neural gene (Sox-2) in excised animal caps (419). Nonetheless, there is further evidence of the importance of L-type calcium channels in neural specification. Striking periodic calcium pulses have been observed at the presumptive site of neural induction (305). It had already been observed in the salamander using aequorin imaging that calcium levels increase dorsally as gastrulation approaches, subsiding as the neural tube begins to form (302); these changes were seen only when aequorin had been microinjected into dorsal, not ventral, blastomeres. Using aequorin imaging in Xenopus, episodic calcium pulses were seen to be superimposed upon this gradual dorsal rise and fall in calcium concentration (305). Large transients began to appear at stage 9 (Figure 11). At stage 10-10.5 regions of increased calcium encompassed groups of between 4 and 35 cells located on a meridian (animal polevegetal pole) at the midline on the dorsal side, around $45^{\circ} \mathrm{N}$ of the equator. The occurrence of transients increased from 4 at stage 8 to 84 at stage 11, localized to an area between 24 and 38 cells above the blastopore lip (anterior dorsal ectoderm). By stage 13, the transients were occurring in what had become the neural plate. The absolute magnitude of the transients was bimodally distributed, with around $15 \%$ of transients of ten times the magnitude of the remainder, with no obvious correlation between magnitude and time of occurrence.

By stage 11, the clusters of cells generating calcium transients has begun to propagate long range calcium waves that spread first laterally, then posteriorly across the dorsal ectoderm with a wave velocity of around $10 \mu \mathrm{m} . \mathrm{s}^{-1}$ (305). Calcium concentrations were estimated to reach $1 \mu \mathrm{M}$ and remained significantly above background for perhaps 20 min after the wave 
had passed. No calcium signals were seen when aequorin was injected ventrally. The calcium transients and calcium waves were completely abolished by treatment with the Ltype calcium channel antagonists $\mathrm{R}(+)$ BayK $8644(10 \mu \mathrm{M})$ or nicardipine $(500 \mu \mathrm{M})$. With $\mathrm{R}(+)$ BayK 8644 , around half of the treated embryos failed to gastrulate; the other half were significantly $(70 \%)$ shorter than control embryos and showed lack of anterior structures such as eyes and melanophores. With nicardipine, all embryos underwent gastrulation and appeared similar in appearance to those BayK-treated embryos that had undergone gastrulation.

The expression of two early expressed neural genes (Zic3, geminin) was determined to assess the effects of the antagonists on neural induction. Expression of both genes was substantially reduced in gastrulating embryos treated with either antagonist (305). In addition, noggin-treated animal caps failed to upregulate NCAM (a broad neural marker) when exposed to R (+)BayK 8466 (305).

These experiments strongly support a role for L-type calcium channel-derived calcium signals in neural induction.

v. Calcium and neural induction - putting one and two together: The three key papers that describe calcium signaling during gastrulation appear discrepant. The one paper showing clear effects on neural gene expression (305) is apparently contradicted by two papers that show: (i) that calcium waves during gastrulation are required for cell movements during convergent extension, but not gene expression (586); (ii) that L-type calcium channels are essential for dorsoventral specification of mesoderm and dorsal competence, but not expression of neural genes (419). My feeling is that resolving these contradictions is straightforward and will produce a broad and useful picture of the contribution that L-type calcium channels make to axis specification and neural induction. Let's start with the concordance between the studies. Two agree that resting calcium concentrations in dorsal regions (ectoderm (305); mesoderm (419)) are substantially higher than in corresponding ventral regions. The third (586) did not attempt this comparison. The same two studies agree that calcium waves arise by simultaneous calcium increase in a cluster of cells, that then propagate tens of cell diameters at 5-10 $\mu \mathrm{m} . \mathrm{s}^{-1}$, that these clusters are randomly distributed within the active tissue, that clusters often fail to initiate a calcium wave and that they are seen only in dorsal tissue (ectoderm (305); mesoderm (588)). Again, the third did not attempt to visualize calcium waves (419). All three studies noted that blocking calcium waves prevented convergent extension/gastrulation. Depleting calcium stores suppressed convergent extension in Keller explants (586); L-type calcium channel inhibitory toxins completely suppressed gastrulation (419), while BayK suppressed gastrulation in half the embryos (305). The remaining embryos and those treated with another blocker, nicardipine, were substantially (50-60\%) shorter than control embryos (305). The toxins are the most robust blockers of L-type channels. It appears that inhibitors of increasing potency arrest gastrulation more effectively. Two studies demonstrated that inhibition of L-type channels led to loss of markers associated with either neural (305) or somite (419) induction by the Spemann Organizer. The third showed that depleting intracellular calcium stores blocked convergent extension, but not mesodermal gene expression, a disparity to which we shall return. It was noted that the mesodermal calcium waves would not be readily observable in whole embryos because the mesoderm in the gastrulating embryo is occluded by opaque and pigmented ectoderm and stated that, in Keller explants, calcium waves could be seen in both ectoderm and mesoderm (586) The other reported discrepancy is that animal caps from embryos overexpressing the $a_{1}$ subunit of the L-type calcium channel did not show any induction of the neural gene Sox-2 (419). However, as the authors themselves note, the subunit was expressed poorly in animal cap ectoderm, while others report that L-type 
channels are clearly present in native embryos in comparable quantities in both ectoderm and mesoderm (122).

The overall picture then is of two sets of calcium waves at gastrulation, one set propagating in mesoderm and originating in mesoderm close to the dorsal lip of the blastopore (586), the other originating in dorsal ectoderm in the animal hemisphere and propagating through ectoderm towards the blastopore (305), antiparallel to the mesodermal calcium waves in the underlying tissue. The waves are accompanied by contraction (586). Blocking the waves either by using L-type calcium channel blockers or by emptying intracellular calcium stores prevents convergent extension/gastrulation $(305,419,586)$. It is evident that these calcium waves rely on both L-type calcium channels and calcium release from intracellular stores and that they coordinate the convergent extension cell movements that occur at gastrulation in both ectoderm and mesoderm; from around half way through gastrulation, ectodermal and mesodermal convergent extension movements are well coordinated and the movements of the ectoderm rely on vertical signals from the underlying mesoderm (136).

When it comes to calcium signals and gene expression, my observation is that the calcium waves themselves are a distraction; indeed, the waves can be suppressed by calcium store emptiers without affecting expression of somite marker genes (586). It is clear that L-type calcium channels determine the expression of both early $(419)$ and late $(305,419)$ dorsal genes. My hypothesis is that dorsal gene expression correlates not with calcium waves, but with the higher levels of resting calcium associated with dorsal tissue $(419,586)$ brought about by expression of L-type channels. It is possible that the short, non-propagating calcium pulses in both mesoderm (586) and ectoderm (305) may also play a part in gene expression, though their frequency has been shown to be reduced after depleting calcium stores, while dorsal gene expression is not (588).

vi. Calcium and organogenesis: Aequorin luminescence reveals calcium transients in the kidney anlage after neurulation (122). Kidney tubules will develop in animal caps treated with activin and then retinoic acid. When retinoic acid is added to the caps, calcium signals ensue. The calcium ionophore A23187 and ammonium chloride, two agents that elevate calcium in activin-treated caps, can substitute for retinoic acid in stimulating the appearance of kidney tubules. Activin/retinoic acid treated animal caps do not form kidney tubules when treated with the calcium chelator BAPTA (122). Xenopus expresses a homologue of the polycystin (PKD1) gene (167) that is involved in calcium sensing in the kidney and in kidney morphogenesis (see section VIIB1). It is very possible, though not proven that calcium signaling through the cilia/polycystin mechanism is a key regulatory event in kidney morphogenesis (122).

vii. Calcium and specification of neurotransmission in the neural plate: Neurones in the developing neural plate of Xenopus embryos display spontaneous calcium spikes some time before synaptic connections are established and are slow relative to calcium action potentials, with durations of the order of 10s and frequencies of around 1-5 per hour (190). Different neuronal cell types exhibit different spike frequencies. A recent study has uncovered the unexpected finding that spike frequency determines the class of neurotransmitter expressed and released by the cells (48). In unperturbed neural plate, neuronal cell types with low spike frequencies expressed excitatory neurotransmitters (glutamate, acetylcholine) and those wioth high spike frequencies, inhibitory neurotransmitters (GABA, glycine). The spike frequency was manipulated either by expression of the inward rectifier channel (Kir) to reduce spike frequency or by expressing the TTX-sensitive sodium channel to increase spike frequency, as well as by varying the extracellular calcium concentration. High imposed spike frequencies switched neurotransmitter expression in cells from excitatory to inhibitory, while low imposed spike 
frequencies promoted a switch from inhibitory to excitatory neurotransmission. These effects could be demonstrated in dissociated neural plate cells, as well as in situ (48). What is most striking is that neurotransmitter switching occurred in cells whose developmental cell fate was unperturbed, as judged by the expression of well-established neuronal cell markers, as well as the position and morphology of the cells.

This example of calcium signaling in embryogenesis takes us beyond cell fate specification into the realm of neuronal plasticity, perhaps implying the existence of a homeostatic mechanism to ensure that transmitters are expressed correctly not only in the context of neuronal cell fate but also taking account of the functional neuronal context (48).

viii. Calcium and dorsal patterning in Xenopus: There are thus two calcium signaling systems with very different roles during dorsal patterning: the Wnt/Ca pathway acting through protein kinase C, CaMKII and calcineurin/NF-AT (457) and the L-type calcium channel pathway $(305,419)$, putatively acting through calcineurin/FKBP (633). Recall at this juncture the three phases of dorsoventral patterning. The first phase involves induction of mesoderm by the activin/FGF signaling systems, the second the generation of the Nieuwkoop centre in dorsal mesoderm by Wnt/BMP signaling and the third induction of neural tissue, somites and organs by the Spemann organizer at gastrulation. There is no evidence that calcium signals play a role in the first phase. In the second phase, the $\mathrm{Wnt} / \mathrm{Ca}$ pathway acts ventrally to suppress dorsal signals generated by canonical Wnt signalling, acting in the same sense as the BMP pathway. In the second phase, L-type calcium channels act dorsally to specify dorsal gene expression and propensity for convergent extension movements (collectively dorsal competence) and antagonize the BMP pathway. In the third phase, calcium channels mediate induction brought about by signals from the Spemann Organizer. The molecular architecture of the signaling pathways that lead to calcium channel activation in the second and third phases is very unclear. Given that the current view is that neural/somite cell fates are a default pathway repressed by BMP signaling (201), it is possible that activation of L-type calcium channels in neural and somatic tissue that will give rise to muscle, nerve and bone is simply the beginning of expression of a differentiated phenotype.

The Wnt/Ca pathway probably antagonizes convergent extension movements at gastrulation by signaling in the second phase to prevent the emergence of dorsal comnetence. The Planar Cell Polarity pathway is known to control convergent extension (379), but the link between this pathway and the large scale calcium waves that coordinate convergent extension movements in both Dorsal ectoderm and dorsal mesoderm has not yet been discovered.

The final point to make about calcium signals in Xenopus is that they are very hard to see. The mesodermal calcium waves observed in Keller explants are undetectable in whole embryos, masked as they are by the opaque overlying ectoderm. The absence of signals detectable in the whole embryo does not imply that such signals do not exist.

3. Zebrafish-A number of features of its development recommend the zebrafish as an experimental model, for example, its rapidity. Paramount in the context of this review is the embryo's unusual transparency $(496,596)$, in marked contrast, as we have seen, to the frog. A much greater range of developmental calcium signals has been observed in zebrafish embryos than in any other. Transparency is an important factor, but so too is the use of luminescent imaging, whose advantage over confocal imaging, the ability to image embryos frequently over long periods of time without damage, outweighs a disadvantage, that of lower spatial resolution (596). In addition, though zebrafish are not well-suited to reverse genetics, mutagenesis has identified many mutants defective in developmental patterning. 
i. Cytoplasmic movements: Just as in ascidian, frog and possibly mammalian oocytes, zebrafish eggs undergo a profound cytoplasmic rearrangement after fertilization. Cytoplasm previously arranged in a thin rim around the surface of the oocyte and within the central yolk migrates animal-polewards to form the blastodisc from which the body parts of the embryo will form, a process known as ooplasmic segregation. In the medaka (a teleost closely related to zebrafish) cytoplasmic movements towards the animal pole are actinbased, blocked by cytochalasin D (598), while intracellular membrane-bound compartments are carried towards the animal pole by microtubules (7). Segregation is brought about by a cortical contraction wave that moves from the animal pole downwards towards the equator. Contraction is accompanied by a slow cortical calcium wave that moves towards the equator then stops at the margins of the forming blastodisc at the single cell stage, transforming into a stationary ring of elevated calcium that is co-incident with a ring of contracting actin (103, 319,320 ). Ooplasmic segregation is prevented by microinjection of dibromo-BAPTA (319); the animal pole microtubule array is disrupted, suggesting that the calcium wave may orient the array (8).

ii. Cell migration in Zebrafish and the development of the body plan: The stages of zebrafish development have been set out definitively by Kimmel (261). Cell division in the segregated cytoplasm gives rise through variations in cleavage plane orientation to a 32-cell embryo that generally presents as a $4 \times 8$ array. The next division plane is horizontal, giving rise to two types of cells: those that are superficial and those that are deep, covered by superficial cells. The superficial cells are known as the enveloping layer (EVL) and the deep layer cells as DEL cells. It is the DEL cells that give rise to embryonic structures. The EVL cells play important roles in morphogenesis, but ultimately form periderm, a protective single cell layer that covers the developing embryo. The EVL and DEL cells now form a blastodisc atop the yolk cell. At cycle 10 (1024 cells), at about the time of the midblastula transition, a third cell layer arises. The marginal tier of blastomeres undergoes a dissolution of their cell membranes to become syncytial with the underlying yolk cells, becoming the yolk syncytial layer (YSL). The YSL develops as a ring beneath the blastodisc, but spreads to become a continuous sheet beneath the blastodisc (the I-YSL). It is thought that the I-YSL is responsible for delivering nutrients from the yolk cell to the embryo. A small portion of the YSL remains external, just beneath the EVL cells and this E-YSL appears to be the major motor for epiboly. Epiboly, a thinning and spreading of the YSL and blastodisc over the whole surface of the yolk cell, has been aptly described by Kimmel as akin to pulling a knitted ski cap down over the head towards the nape and chin. The E-YSL advances across the yolk, appearing to pull the EVL and DEL behind it. At 50\% epiboly, that is, when the YSL margin has reached the equator, gastrulation begins. Involution in teleosts involves the ski cap hem rolling back under itself. The DEL cells at the margin that begin to move back upwards under the overlying cells are known as the hypoblast; cells that remain on the surface are known as the epiblast. The border between the hypoblast cells moving upwards and the epiblast cells at the surface is Brachet's cleft, by analogy with Xenopus gastrulation. The epiblast corresponds to epidermal and neural cell fates, while then hypoblast is endomesoderm, there being no obvious differentiation at this stage in teleosts between mesoderm and endoderm. Convergence movements occur at gastrulation to produce a dorsal thickening equatorially (the embryonic shield), an accumulation of DEL cells that act as the equivalent of the Spemann organizer in Zebrafish. Extension movements then begin within the embryonic shield to produce the anterior-posterior axis. During gastrulation epiboly is suspended. It resumes as the shield thickens and extends anteriorwards. Thereafter the dorsal structures begin to resolve with the underlying hypoblast into the vertebrate body plan. From here on, developmental patterning is very similar to that of the frog embryo, except that neurulation and segmentation occur in parallel in zebrafish, not sequentially as in the frog. 
iii. Axis formation: It is zebrafish embryos that have provided the necessary demonstration that the weaker Wnts directly modulate calcium signals. Using either confocal fluorescence imaging or luminescence imaging, sporadic calcium transients are observed very early during the cell divisions of the blastodisc $(442,596)$ (Figure 7) and persist until the sphere stage. Spikes in adjacent cells are correlated; aequorin luminescence observations have suggested that they take the form of intercellular calcium waves that propagate through gap junctions which are abundant in blastomeres (596). Transients are seen mostly in EVL cells, with some activity in the I-YSL. There are no indications that the occurrence of spikes varies along the dorso-ventral axis $(442,496)$. Inositol trisphosphate levels in the embryo increase after the 32 cell stage (442). Expression of XWnt-5a in zebrafish doubles the frequency of the sporadic transients and enhances their synchrony with the cell division cycle (496).

Expressing a rat Frizzled-2 receptor in zebrafish embryos also enhances the endogenous transients (495). Enhanced calcium signalling with expression of either ligand (Wnt) or receptor (Rfz-2) implies that each can interact with an endogenous, analogous receptor or ligand and suggests that an endogenous Wnt/Frizzled calcium-linked may be responsible for the endogenous transients. However, though the trimeric G-protein inhibitors pertussis toxin, GDP- $\beta$-S or a-transducin prevented the enhancement of the endogenous spikes by both XWnt-5A and RFz-2, they did not affect the endogenous transients (495).

This observation in zebrafish embryos is a key element in understanding the relevance of the $\mathrm{Wnt} / \mathrm{Ca}^{2+}$ in frog embryos, so it is important to determine whether the overall features of the dorso-ventral specification mechanism are the same. It has been done. Lithium dorsalizes zebrafish embryos, as it does frog (39), indeed microinjecting lithium into 16-cell embryos reveals a pre-existing dorso-ventral polarity (1). These effects are rescued by myo-inositol. When the experiments that expressed exogenous 5-HT receptors to stimulate phosphoinositide signalling in the frog (21) are repeated in zebrafish, a similar ventralizing phenotype is obtained that also phenocopies XWnt-5A overexpression (496). The effects of ectopic RFz-2 expression on the sporadic calcium spikes can be blocked with the pharmacological inhibitor that targets inositol monophosphatase (L-690330), as lithium may; the block is relieved by myo-inositol (495). L-690330 also reduced the frequency of endogenous sporadic calcium spikes, an effect rescued by myo-inositol, and caused dorsalization (600). In experiments paralleling those in Xenopus (457), an $\mathrm{InsP}_{3}$-receptor antibody and the $\mathrm{InsP}_{3}$-receptor antagonist Xestospongin $\mathrm{C}$ induced dorsalization phenotypes in zebrafish (600). The phospholipase C $\beta$ inhibitor U-73122 induced dosedependent mild to severe dorsalizing phenotypes, while its inactive congener U-73343 was without effect (600). The calcium store depleter thapsigargin also induced dorsalized phenotypes with high penetrance, with marked expansion of the chordin expression domain. Xestospongin $C$ treatment expanded the region of expression of $\beta$-catenin, as did U-73122. Both treatments expanded the expression domains of a homeodomain protein bozozok, downstream of $\beta$-catenin (600). Injection of the Xenopus Dsh construct enhanced sporadic EVL calcium transients and the XDsh $\triangle$ DIX construct that stimulates the non-canonical Wnt pathway was even more effective (484). This finding demonstrates explicitly that the Wnt/ Ca pathway defined in Xenopus modulates calcium signaling.

Isolation of zebrafish mutants defective in dorsal competence and specification confirms the contribution of the Wnt/Ca pathway to dorsoventral specification. The silberblick mutation results in abnormal extension, leading to midline defects including the development of a single eye; the mutation can be rescued by a Dsh $\Delta$ DIX construct, demonstrating a contribution from the non-canonical Wnt pathway to convergent extension in zebrafish (207). Silberblick encodes a weaker Wnt (zWnt11). zWnt 11 overexpression markedly increased the frequency of EVL calcium transients (599). Zebrafish Wnt 5 is encoded by the 
pipetail gene. Pipetail mutants display mild defects such as shortened body length and malformation of the tail implying an effect on convergent extension, but the phenotype of a pipetaill silberblick double mutant shows genetic interaction, with a dorsalized phenotype markedly more severe than the sum of the parts (599). Blastodisc calcium transients are very much suppressed in pipetail/zWnt 5 mutants, particularly in the I-YSL where they disappear completely. Conversely overexpression of zWnt 5 mRNA enhanced EVL calcium transients in the period up to the mid-blastula transition while zWnt 5 DNA enhanced the transients after the MBT when expression of zygotic genes begins (599). zWnt 5 overexpression from mRNAor microinjection led unexpectedly to hyperdorsalization $(260,600)$. This was interpreted as a consequence of calcium store depletion due to the enhanced calcium release before the MBT $(599,600)$ which would suppress the Wnt/Ca pathway ventrally. Support for the interpretation was obtained by overexpressing zWnt 5 from DNA: this approach led to the expected cell movement defects and hyperventralization (599). It it was elegantly demonstrated by using zWnt 5 DNA injection to rescue $p p t^{-/}$embryos during early development that the relatively mild pipetail phenotype is due to the presence of maternal gene product before the MBT: homozygous mutant embryos from homozygous mutant females showed very severe dorsalization, including axis duplication, due to marked expansion of the $\beta$-catenin expression domain and extended and ectopic chordin expression (599)

Slusarski's study (599) is a key paper in the field in two important respects: (i) it provides the only direct evidence that the absence of endogenous Wnt/Ca activity causes both dorsalization and suppression of calcium signals in the blastula; (ii) it demonstrates two epochs of Wnt/Ca signaling in that before the MBT, the major action of the pathway is on antagonizing dorsal competence, while after the MBT, the pathway is controlling convergent extension movements. However, the study did not assess the frequency of calcium transients in homozygous mutant embryos from homozygous mutant females - a key experiment needed to determine whether the suppression of calcium signals correlates with antagonism of dorsal competence before the MBT.

As we have discussed, it is thought that convergent extension movements in Xenopus are controlled by the Planar Cell Polarity (PCP) pathway. The clearest evidence that the PCP and $\mathrm{Wnt} / \mathrm{Ca}$ pathways interact to control convergent extension comes from studies on the zebrafish homologues of the prickle ( $p k$ ) gene of Drosophila, a component of the PCP pathway. There are two $z P k s$ (578). I shall discuss $z P k 1$, as it has the stronger phenotype. It is expressed dorsally and axially. Antisense morpholino mRNA directed against zPk1strongly suppresses convergent extension movements, but not the expression of dorsal marker genes; this effect is not apparently cell autonomous. Exogenous Pk1 activates AP-1 transcription, as would be expected if it signals via the PCP (JNK) pathway; it also enhances calcium signals in blastodisc EVL cells (578).

The evidence for the involvement of calcium signalling in the antagonism of the canonical Wnt pathway to specify the ventral embryonic developmental field is thus persuasive. The data from zebrafish complement and confirm those obtained in Xenopus (Figure 13).

However, beyond the direct demonstration that weaker Wnt signaling can modulate calcium transients in the blastula and the evidence that PCP signaling can also modulate these transients, we have learned little more about the Wnt/Ca pathway. Most confusingly, while there is abundant evidence that interfering with calcium signals ventrally promotes dorsal cell fates, there is no evidence at all from these studies using fluorescent calcium reporter dyes that the blastodisc calcium signals show any dorsoventral differences $(484,495,496$, $578,599,600)$. There is one report using the luminescent reporter aequorin that has been said to show that calcium levels are higher ventrally than dorsally in the blastodisc, but in fact a higher calcium in the presumptive ventral region was observed in only four of seven 
embryos; in two others, no difference was observed and in the seventh, calcium was higher dorsally (103).

\section{iv. The virtues of the luminescence method for detecting embryonic calcium signals:}

The photoprotein aequorin emits light as it reacts with calcium; the rate of light production is steeply dependent on calcium concentration. The use of aequorin together with imaging photon detectors has proved very fruitful in revealing calcium signals during zebrafish development $(103,172,596)$. Imaging photon detectors record as a long list the times and positions of the arrival of individual photons emitted by aequorin. Images of aequorin emission are then built up by choosing two points in time, then plotting the events in a two dimensional array. This is very different from fluorescence imaging, where images are captured at fixed intervals with fixed sampling times. The fluorescence imager always faces a dilemma: to sample as quickly as possible, but to expose the embryo to the phototoxic effects of the exciting light; or to sample infrequently, sparing the embryo, but running the risk of missing events in the long intervals between samples. Aequorin is self-luminous, requiring no exciting light; the imaging photon detector allows continuous sampling. After the experiment is completed, the pattern of light emission with time can be inspected, intervals that show calcium increases in the whole embryo determined and images assembled of the intervals of interest. The technique has captured focal areas of higher calcium concentration (hotspots) and widespread calcium waves in zebrafish embryos. The method has a disadvantage: levels of photon emission from aequorin are too low and the optical configuration insufficiently resolved to determine calcium concentrations at the single cell level in all but the very large cells of the early blastodisc. However, once the aequorin-derived data had given an idea of where and when to look, it has been possible to detect calcium waves in early zebrafish embryos using multiphoton microscopy (171) and it may be possible to extend multiphoton fluorescence imaging to the cellular level.

The two key papers $(103,172)$ used different approaches to analysis, a difference reflected in their findings. Jaffe's (103) approach was to build up static images over fixed and relatively long time intervals. The temporal averaging over these long time intervals would tend to produce images that reflect temporally-stable calcium patterns within the embryo. Miller's approach (172) was to use long time integration intervals, but to space images temporally at intervals shorter than the integration interval; this approach would be more likely to detect spatiotemporal element of embryonic calcium signaling.

Using a very sensitive aequorin that might be expected to reveal small changes in resting calcium as well as calcium transients, Jaffe found that average calcium levels in the zebrafish embryo rose after fertilization, dipped mid-blastula, rose again to peak just before half way through gastrulation and finally rose substantially early in the segmentation stage (103). This overall pattern might be taken to reflect the overall intensity of calcium signaling during zebrafish development.

v. Aequorin imaging in the blastodisc: Aequorin imaging $(103,172)$ confirms the existence of sporadic calcium transients in the EVL and the absence of any obvious dorsoventral gradient $(442,495,496)$. In addition, two other signals were detected: a mid-blastula calcium wave that originated at the animal pole and a hotspot associated with marginal blastomeres that may be involved in triggering dissolution of the first tier of marginal cells to form the YSL.

vi. Aequorin and epiboly and gastrulation: The more static images of epiboly showed that by $75 \%$ epiboly, calcium was high at the blastoderm margin (E-YSL); there was a clear dorso-ventral gradient of calcium around the margin, with dorsal regions showing the highest calcium concentrations (103). The more dynamic images revealed three patterns of 
calcium transients between 50 and $75 \%$ epiboly $(172,596)$. At the shield stage a small ventral region at the equator opposite to the shield showed elevated calcium levels 6-8 fold higher than the rest of the embryo. This calcium signal is very similar to one described in medaka (Oryzias latipes) from which calcium waves emanante to induce contraction of the EVL during epiboly $(27,155)$. The calcium wave in medaka arises at a ventral pacemaker and is carried by cells of stellate shape just beneath the EVL and can be seen in EVL explants (494). No such waves were reported in zebrafish. At around 65\% epiboly, the remaining exposed surface of the yolk cell bounded by the YSL showed a brief, large and uniform calcium imcrease (Figure 14). This yolk flash appears to be very superficial and so perhaps brought about by a calcium influx. Additional hotspots appeared around the blastoderm margin, one located dorsally; these hotspots then generated calcium waves that traveled around the margin. Miller has termed these gastrulation waves (172). Until blastopore closure, their average frequency was 7 per hour, increasing to 11-12 per hour after closure of the blastopore. By $85 \%$ epiboly, these waves almost always originated from a hot spot at the dorsal midline. The waves propagated decrementally at a velocity of around $5 \mu \mathrm{m} . \mathrm{s}^{-1}$. In addition, by $85 \%$ epiboly, waves from the dorsal hotspot also moved up the anterior-posterior axis of the embryo towards the animal pole; axial and marginal waves were not observed to occur at the same time, leading to the suggestion that the dorsal hot spot could originate waves only in one or other of the two routes (172). If resting calcium concentrations were assumed to be around $100 \mathrm{nM}$, then the peak level in the hotspot during wave initiation was estimated to be $1 \mu \mathrm{M}$. Interestingly, wave initation at the hotspot was preceded by a widespread and quasi-synchronous small calcium increase across much of the embryo. A hotspot persisted dorsally after blastopore closure and became incorporated into the developing tailbud, just rostral to the blastopore.

vii. Aequorin and segmentation and organogenesis: The long range cell migrations of gastrulation establish the embryonic body plan, then give way to local inductive interactions that pave the way for organogenesis. There is a marked increase in resting calcium across the whole embryo during early segmentation and an increase in the frequency of calcium spikes (103). Dynamic aequorin imaging has detected successive calcium pacemaker regions during segmentation and organogenesis $(103,596)$. The first is seen in the tail bud as epiboly is completed; it is probably the same locus as that which generates the gastrulation waves. Waves continue to propagate from the tail bud at around one every $5 \mathrm{~min}$. This activity then ceases. A second locus of activity is observed in the developing brain, perhaps at the midbrain-hindbrain boundary. Static aequorin imaging shows a small disparity in calcium concentration across the midbrain/hindbrain boundary, with forebrain and midbrain showing a consistent $30 \mathrm{nM}$ higher calcium concentration than hindbrain (103). Waves are no longer observed, but calcium pulses also appear in the optic region at the late somite stage (596). A third locus is seen at the early somite stage in the trunk region, correlating with somite formation. (596). Static aequorin imaging detects a clear antero-posterior gradient of calcium with calcium highest at the posterior, apparently enhanced by a slow migration of a high calcium zone in the trunk that moves slowly backwards from 10-14 hours of development (103). Microinjection of low concentrations of the mild calcium chelator dibromo-BAPTA gives rise to small eyes and bad hearts, with poorly developed atria, ventricles incapable of pumping blood and an enlarged pericardium (103).

viii. Calcium signals in zebrafish embryos: Experiments on calcium signaling in zebrafish development thus offer two very different perspectives. On the one hand, experiments involving components of the Wnt/Ca pathway argue strongly that calcium signals are essential for dorsoventral specification and convergent extension, as in the frog. But experiments demonstrating effects of weaker Wnt agonists and antagonists on calcium signaling have been made only on the EVL calcium transients in the blastodisc. These EVL 
transients are seen in tissue that forms no part of the embryo itself and show no obvious dorso-ventral patterning. On the other hand, aequorin imaging has revealed a rich tapestry of developmental calcium pulses and waves that occur at times and in places that testify to their likely developmental significance, but we are completely ignorant of their links to known developmental signaling pathways.

It is possible to make one or two conjectures. The ventral calcium hotspot that appears at shield stage when the dorso-ventral axis first becomes apparent $(172,594,596)$ may be a manifestation of the Wnt/Ca pathway. It is also possible that the panembryonic small increase in calcium that just precedes the emanation of a gastrulation wave from a hotspot (172) may reflect the calcium channel/propagating wave sequence seen in Xenopus embryos (see Section VA2 v). It has been pointed out $(595,596)$ that some experiments in chick embryos (which are more amenable to experimental manipulation at the somite/neurula stage) are consistent with the idea of developmental calcium signals. For example, application of the calcium ionophore A23187 to chick embryos at the appropriate stage induces somites while embryos treated with the calcium channel blockers verapamil and papaverine do not undergo segmentation (78); the same treatments had analogous effects on folding of the neural tube (149); optic cup formation is known to be a calcium-regulated process (49). Similarly spontaneous calcium transients have been shown to be essential for the early differentiation of Xenopus myocytes (148).

There is an obvious need for mechanistic experiments in zebrafish.

\section{B. Calcium and left-right axis formation}

1. Mouse embryos and the role of ciliary calcium signals-The left-right body plan in vertebrates is determined by left-side expression of genes, for example nodal and lefty (368), that encourage development of leftward structures. The question is how bilateral symmetry is broken, a question analogous to that of how dorsoventral asymmetry is set up. We know the answer to the latter question in vertebrates: the fertilizing sperm provides an asymmetry that is exploited by cytoskeletal rearrangements (Section VA2). Left right axis determination involves no external agent. Instead it exploits molecular chirality in an intricate and delicate way.

Mutations in single genes can disrupt left-right axis formation. In Kartagener syndrome, half of the affected individuals (there are other phenotypes) have reversed left right body plans (cited in 361). A similar pattern is seen in mouse $i V$ and $P k d 2$ mutants. Kartagena syndrome patients have immotile cilia (10), IV mice carry a point mutation in the left-right dynein (lrd) gene (532) and $P k d 2$ mice lack a ciliary calcium channel (424). All this points to the involvement of cilia. The body of evidence pointing to the need for cilia in left-right axis formation has been set out by Tabin (544).

Left-right axis formation occurs across a structure called Hensen's node. A key observation in the field was that fluid flowed from right to left across the node, propelled by ciliary motion and so absent in mice lacking cilia (405); a second was that the left-right randomization phenotype in iV/iv mice could be rescued by applying fluid flow to Hensen's node from right to left and the left-right axis reversed in wild type mice by applying fluid flow from left to right across the node (404). These and other similar observations firmly established that fluid flow across the node was a determining event in left right axis formation (544). The basis of the symmetry-breaking event is thus the unidirectional flow of fluid across the node caused by the asymmetric ciliary beat pattern that in turn depends on the chiral properties of tubulin. 
Tabin set out the hypothesis (Figure 15) that there were two sets of cilia in the node, motile cilia and sensory cilia (544). He noted that sensory cilia would produce a calcium signal when bent and that the calcium signal, amplified by calcium-induced calcium release could propagate from cell to cell via gap junctions (436). $P k d 1$ and $P k d 2$ are genes that are essential for kidney development; homozygous mutations are embryonic lethals and human heterozygotes develop polycystic kidney disease as adults, one of the most common forms of kidney disease $(167,338,508)$. The genes encode a calcium channel localized to primary cilia and responsible for sensing fluid flow in kidney epithelium (167, 340, 398, 590). Tabin predicted that fluid flow from right to left at the node generated by motile cilia would stimulate sensory cilia on the left, but not right, of the node and generate a calcium signal localized to the left of the node (544). Almost immediately, a study proved that this was the case (361). It showed that all cilia at the node expressed the $P k d 2$ product, polycystin- 2 but that only a central subset of cilia expressed the lrd protein and so were motile. Intracellular calcium concentrations measured with Fluo3 were substantially higher of the left hand side of the node, often over a region comprising several cell diameters; this difference was absent in homozygous mutants lacking either lrd or polycystin-2. In addition, fluid flow transports particulate cargo containing FGF and retinoic acid that appears to be responsible for the increased intracellular calcium concentrations in the cells at the left side of the node (550). Retinoic acid has also been implicated in left-right axis formation in zebrafish (253). These experiments are a compelling demonstration that fluid flow at the node leads to a differential intracellular calcium signalling across the node. They do not themselves demonstrate that this calcium signal alters gene expression. This comes next.

\section{Chick embryos and the role of calcium signals in gene expression-Leftness} in vertebrates is determined by an evolutionarily-conserved expression of Nodal in left lateral plate mesoderm (368) which in turn requires an early expression of the gene in endoderm bordering Hensen's node. Nodal expression of Nodal involves the notch signaling pathway in both mouse and zebrafish $(282,439)$. In the chick, expression of the notch ligand DII1 evolves on the left, but not right, side of Hensen's node due, it is inferred by modelling, to a preexisting left-right asymmetry (440). One possible asymmetry is the left-right gradient in membrane potential and $\mathrm{H}^{+} / \mathrm{K}^{+}$ATPase antiporter activity (324). Exogenous expression of $\mathrm{H}^{+} / \mathrm{K}^{+}$ATPase together with potassium channel subunits (Kir4.1) that would be expected to perturb membrane potential led to laterality defects. Inhibition of the $\mathrm{H}^{+} / \mathrm{K}^{+}$ATPase disturbed left-right patterning and Notch expression (440). Strikingly and quite unexpectedly, a left-right gradient of extracellular calcium concentration has been demonstrated in the chick node, dependent on $\mathrm{H}^{+} / \mathrm{K}^{+}$ATPase activity (440) and so correlated with membrane depolarization in the left nodal region and with Notch expression. Perturbing the asymmetry with local calcium or BAPTA treatments and with $\mathrm{a} \mathrm{H}^{+} /$ $\mathrm{K}^{+}$ATPase inhibitor leads to the expected perturbations of left-right patterning. It is suggested that the asymmetry in extracellular calcium concentration leads to altered binding of DII1 to its Notch receptor (a conjecture substantiated in vitro), thus explaining the laterality of gene expression (440). It is conjectured that the lateral gradient of extracellular calcium is due to its electrophoretic accumulation on the left of the node as a consequence of a right to left extracellular current generated by the lateral difference in cellular membrane potentials $(440,445)$.

3. Calcium and left-right symmetry breaking-Putting the findings in mouse and chick together, the simplest hypothesis is that the intracellular calcium signaling in the left of the node as a result of fluid flow and sensing by primary cilia leads to modulation of $\mathrm{H}^{+}$/ $\mathrm{K}^{+}$ATPase activity with depolarization of the left anlage, as predicted by Tabin (544). This causal chain links right to left fluid flow with gene expression. A counterargument is that asymmetries in $\mathrm{H}^{+} / \mathrm{K}^{+}$ATPase are seen very early in development, at the 2-4 cell stage in 
Xenopus (324); moreover $\mathrm{PKC} \gamma$ signaling in Xenopus specifies left-right asymmetries early in gastrulation, before the appearance of monocilia (281). This line of thinking implies that left-right asymmetry may be specified very early in development. However, the early asymmetry in $\mathrm{H}^{+} / \mathrm{K}^{+}$ATPase expression disappears in the early cleavage stages in Xenopus; no such asymmetry is seen in the chick (324) and there is no evidence for PKC $\gamma$ signaling in left-right patterning in chick or mouse (281). Moreover, the development of membrane potential asymmetry coincides with the appearance of motile cilia (142). It seems sensible to stick with the simplest hypothesis for chick and mouse.

The mechanism underlying the extracellular accumulation of calcium on the left side of Hensen's node has not been satisfactorily explained. The measured $20 \mathrm{mV}$ difference in resting membrane potential is unlikely to generate a current sufficient to account for the observed difference in extracellular calcium between left and right peripheries of the node. In addition, an electrophoretic accumulation of calcium requires current to pass not only extracellularly, but transcellularly through gap junctions. Gap junctional communication is essential for left-right determination in both chick and Xenopus embryos $(322,323)$. However, the gap junction subunit connexin 43 is not present in the node itself, but in peripheral endoderm (322). This observation favours the idea that gap junctional communication is essential for the local left side spread of calcium signals generated by monocilia (361); it does not support the idea of transnodal electrophoretic currents (324, 445).

\section{CALCIUM SIGNALS IN EGGS AND EMBRYOS}

\section{Summary}

The point here is two fold. First that the ever changing landscape as development ensues poses special challenges when trying to understand calcium signals: in most other areas of calcium signalling, a reproducible calcium response can be obtained by repeated application of an external stimulus, but during development stimuli are internal, each occurring only once as the developmental programme unfolds. Second that the changing landscape during development manifests very different sorts of calcium signal, spanning the full range of known calcium signalling mechanisms.

There is a fascination that grips all who work with eggs and embryos: that of seeing a developmental programme unfold before their very eyes. This fascination is bought at a price because in any embryo, the same thing never happens twice, the context of any event changing as maturation, fertilization and development proceed. When thinking about calcium signals during development, it is very important to be aware of this analogy between development and history, the difference being that development, unlike history, repeats itself manifold times in the near-identical ontogeny of each individual of a species. It is the stereotypic repetition of the developmental programme that permits the methods of cell physiology to be applied to eggs and embryos but, unlike many other experiments in physiology, the same protocol cannot be applied to the same embryo twice. Moreover, experiments in eggs and embryos are in general aiming at a moving target. It is, I think, no coincidence that we know more about calcium signals at fertilization than at other points in development. It is because the occurrence and timing of the experimental event is under the experimentalist's control through the addition of sperm. If the reader should feel disappointed that often the part played by calcium in a developmental process is tantalizingly obscure, these experimental limitations should be borne in mind. 


\section{A. Development as a showcase for calcium signalling}

I have focused in this article on calcium signals in embryos for which there is an experimental context of mechanistic studies and where there are links to protein signaling networks. There are other phenomenological observations of calcium signals in development, many of which are discussed in Jaffe's relatively recent review (236) and include the role of calcium in polarization of Fucus zygotes; in the stalk/spore cell fate in Dictyostelium, where striking calcium waves are observed during mound formation; in Drosophila eye discs; in plant floret formation.

It will have been noted that while there is evidence during development of the importance of calcium signals in modulating or controlling membrane fusion, the microtubule and actin cytoskeleton, meiosis and mitosis, cell division, cell migration and cell fate, there is a tendency to study one or other of these mechanisms at specific developmental stages. Take, for example, cell cycle calcium signals. Almost all the data linking calcium signals to the control of mitosis and cytokinesis come from experiments on one- or two-celled embryos. Are we to conclude that the role of calcium in mitosis is confined to these large-celled stages? I want to argue instead that calcium signalling during mitosis and cytokinesis is a general property of both germ line and somatic cells and that the large cells of the early embryo offer a showcase for these signals that are otherwise much more difficult to detect with our current methods. My point is that the various stages of development provide different contexts in which to study different aspects of calcium signalling.

1. The fertilization showcase-Fertilization is a showcase for calcium waves and their mechanisms of propagation. It remains the only context in which physiological calcium waves can be ascribed a clear physiological function, the activation of a very large cell by a very small one. The property of generating intracellular calcium waves is particular to mature eggs and oocytes. It arises because a fundamental change takes place in the distribution and properties of the ER. As I have already remarked, as maturation proceeds, ER leaves its juxta-nuclear location, spreading out throughout the cytoplasm, often forming ER clusters at the plasma membrane. I suggest that this redistribution reflects a switch from the local cell cycle calcium signalling of meiosis to the global cell cycle signalling of fertilization. Fertilization is thus also a showcase for the mechanisms through which calcium interacts with cell cycle control mechanisms such as the APC and proteasome-mediated degradation of cyclins. Fertilization is a showcase for calcium-cytoskeleton interactions, responsible for pronuclear migration and ooplasmic segregation in ascidian, sea urchin zebrafish and frog. Finally fertilization is a showcase for the variety of calcium releasing mechanisms: NO, cGMP, cADPr and NAADP as well as $\mathrm{InsP}_{3}$.

2. The cell cycle showcase of the early blastula-After fertilization, the relation between ER and nucleus and spindle becomes again more intimate. Nonetheless the large size and specialization for rapid synchronous cell divisions of the early blastomeres has allowed the identification of calcium signals that control entry into mitosis, chromosome disjunction and cytokinesis. The supposition is that these signals continue to be present even once the small size of the continually dividing blastomeres makes them hard to discern.

3. The axis determination showcase of the late blastula-The late blastula stage of frog and zebrafish reveal a novel role for calcium signals in interactions with the canonical Wnt pathway through modulation of cell movements and developmental gene expression in the control of cell fate. It will offer a model for calcium-Wnt interactions later in development. 
The existence of fate-determining calcium signals in late blastulae raises the question of whether or how calcium signals can have more that one job to do at this stage of development. Can they control cell cycle progression at the same time as they contribute to axis specification? In syncytial blastomeres of Drosophila embryos a dorsoventral gradient of calcium concentration coexists with cell cycle calcium signals (102) ${ }^{1}$. It is interesting to note that one effect of XWnt-5A expression is to bring the late blastula calcium spikes into closer synchrony with the global cell division cycle of the blastula (496), so that they clearly occur at the time of mitosis and cell division. It is possible that this is an effect not of altering the synchrony of spikes and cell division in single blastomeres, but of improving global cell cycle synchrony in the blastula, so that the association of the calcium spikes with cell division becomes more apparent. It may be that the axis formation mechanism is hitching a ride on the cell cycle signals by increasing their magnitude without altering their timing.

4. The zebrafish gastrula as a showcase for intercellular calcium waves-The paradox raised above is, of course, the simplest formulation at a relatively simple stage of development of a broader question pertinent to later development: how can signals control the cell cycle and pattern formation while at the same time also controlling, for example, membrane fusion events and cytoskeletal functions within the same cell? The idea that cells express subsets of the broad set of calcium signaling components (their calcium signaling toolkit) is well developed (41). The answer to the broader question is less obvious, but a clue may be found in the intercellular calcium waves identified in zebrafish gastrula. The function of these waves is presumably to coordinate the calcium signalling systems of individual embryonic cells. Miller (596) has noted an evolution of calcium signal patterning during zebrafish development that parallels at the multicellular level the change from local to global that we have noted at fertilization. In the very early blastomere calcium signals are cell autonomous; in late blastula there are the beginnings of coordination from blastomere to blastomere, with signals spreading one or two cell diameters; at gastrula, full blown intercellular waves appear that travel hundreds of microns; finally during segmentation calcium signals once again become focal. It will be interesting to determine what mechanisms underlie these changes.

\section{B. What calcium signals do}

Summing up, it is worth surveying in the broadest sense what we know about the targets of calcium signalling in eggs and embryos. The answer is, I'm afraid, not a great deal. In eggs oocytes and early embryos the effectors are protein kinase C, CaM Kinase II and calcineurin and the targets are the kinases and phosphatases of the cell cycle control machinery. This level of post-translational control is appropriate for cells whose transcriptional machinery is largely inactive. In late blastula there is evidence of regulation of gene transcription by the same trio: CaM Kinase II, protein kinase C, and calcineurin/NF-AT. We do not know how, or even in most cases strictly whether, the calcium signals of later development shape the pattern of the embryo, so there is much to do (Figure 16).

\section{Acknowledgments}

I should like to thank the following for useful comments and criticisms: Dr John Carroll, Dr Anthony Galione, Dr Laurinda Jaffe, Dr Roberto Mayor, Dr Luigia Santella, Dr Diane Slusarski, Dr Nick Spitzer, Professor Karl Swann. I thank Michael Aitchison for preparing the figures. Work in the lab is supported by BBSRC and the Wellcome Trust.

\footnotetext{
${ }^{1}$ Calcium is observed to higher dorsally than ventrally in Drosophila, which may seem to be at odds with the idea that calcium is higher ventrally than dorsally in vertebrates, until one takes note of the fact that dorsal structures in vertebrates correspond to ventral structures in insects and other non-chordates 19.
} 


\section{GLOSSARY}

Anaphase

\section{Anaphase \\ promoting complex/ \\ Cyclosome}

Animal cap

Anlage

Anterior-posterior axis

Axial expression

Blastocoel roof
explants (BCR)

Blastodisc

Blastopore

Brachet's cleft

Centrosome

Contractile ring

Convergent extension

Cyclin/cdk1

kinase

Cytokinesis

Dorsal lip of blastopore

Embryonic shield the stage of mitosis during which sister chromatids are separated and pulled to opposite poles of the mitotic spindle by microtubules and microtubule motors.

a large molecular machine active at points during the cell division cycle that targets specific proteins for degradation.

an experimental preparation dissected from Xenopus embryos consisting of the ectoderm that overlies the blastocoel (see Figure *).

a developmental field that will give rise to a specific embryonic structure, for example the kidney.

the embryonic axis that runs from head to tail.

gene expression concentration on the midline of the anteriorposterior axis.

the underside of an animal cap.

the cell mass that accumulates at the animal pole during the early development of, for example, zebrafish embryos.

the site of ingression (involution) of cells during gastrulation.

the space between the involuting mesoderm and the overlying ectoderm during gastrulation.

the amorphous cellular organelle that organises the cell's microtubule network and splits to form the spindle poles during mitosis.

a torus of actin and associated myosin that underlies the cleavage furrow during cytokinesis and which shortens like a purse string during cytokinesis.

the cellular behaviour that underlies gastrulation movements; it consists of cell elongation and intercalation and is responsible for the antero-posterior elongation of the embryo during gastrulation.

activation of this mitotic kinase leads to entry into mitosis.

the process of cell division during which the cell membrane is drawn in equatorially by the contractile ring, leading to fission of the two daughter cells.

embryonic tissue situated dorsad and adjacent to the blastopore in Xenopus and thiugh to be the source of dorsal-specifying signals during gastrulation.

a dorsal thickening in zebrafish embryos that appears during epiboly and that is thought to be the source of dorsal-specifying signals analogous to Spemann's organizer in Xenopus. 
Echinoderm

Ectoderm

Egg

Encephalon

Endoderm

Epiboly

Epidermal fate

DEL $=$ deep layer

Dorso-ventral axis

Dorsalizing

Embryonic axes

EVL = enveloping layer

Gastrulation

Germline

Hensen's node

Immature oocyte

Involution

Keller explants

Kinetochore

Left-right axis a phylum that includes sea urchin and starfish, as well as brittle stars, holuthurians and crinoids.

embryonic tissue that will give rise to mesoderm and later to epidermal and neural tissue.

strictly speaking, an oocyte that has completed meiosis.

the developing brain.

embryonic tissue that will give rise to blood, liver, endocrine tissue and so on.

the migration of the blastodisc cells over the yolk layers in teleosts; the equivalent of gastrulation in Xenopus.

ectoderm that becomes epiderm (epithelia and so on), rather than neural tissue.

cells within the teleost blastodisc that ultimately give rise to embryonic structures.

the embryonic axis that specifies the differences between back and belly.

a treatment that enhances dorso-anterior structures in an embryo at the expense of ventral structures; an extreme example is the production of two heads.

three axes that determine an embryos asymmetries; dorso-ventral (back-belly), anterior-posterior (head-tail) and left-right.

cells at the exterior of the teleost blastodisc that enclose the deep layer (DEL) cells; these cells play a prominent part in morphogenesis during epiboly, but ultimately give rise to periderm, an extraembryonic protective layer.

the key event in development that leads to formation of the gut and to the specification of antero-posterior structures (from brain to tail in vertebrates).

embryonic cells that are segregated from other embryonic cells and give rise to sperm and oocytes; all other cells in the embryo are somatic cells.

an embryonic structure at the midline at the level of the developing heart that determines left-right asymmetry.

a female germ line cell arrested in first meiotic prophase.

movement of cells into and through the blastopore during gastrulation.

dissections of the blastopore lip that will undergo convergentextension movements in vitro.

the specialised region on each chromosome that forms the attachment point for microtubules during meiosis and mitosis.

the embryonic axis that determines the asymmetry of internal organs such as liver, spleen and heart in vertebrates, 
MAP kinase

\author{
Mature oocyte \\ Meiosis/Meiotic \\ cell cycle
}

Melanophores

Mesoderm

Metaphase

Neural plate

Notochord

Oocyte

Ooplasmic segregation

Presumptive

Pronephros

Prophase

Proteasome

Segmentation

Spemann

organizer

Sister chromatids

Somites

Syncytial nuclear division a protein kinase activity essential for oocyte maturation and for meiotic arrest prior to fertilization; its activity is also essential for embryonic mitosis.

an oocyte that has undergone meiotic recombination and is competent to be fertilized.

the process peculiar to germ cells that ensures genetic recombination and results in haploid gametes.

pigment cells characteristic of the epidermal developmental lineage.

cells in the embryo that will give rise to muscle an connective tissue.

the phase of mitosis at which chromosome bivalents are captured by the spindle microtubules and constrained by them to align as a disc prior to anaphase; misalignment of chromosomes prevents anaphase onset due to operation of the so-called spindle checkpoint.

the anteroposterior midline structure that gives rise to the nervous system during neurulation.

a dorsal antero-posterior tubular structure that forms as a result of neurulation; it specifies the development of the cephalospinal nervous system in vertebrates.

female germline cell that undergoes meiosis, maturing to produce a fertilizable mature oocyte or egg.

the migration of the embronic cell mass to the animal pole in teleosts.

denotes a cell mass that will subsequently assume a defined fate and develop into an organ, neural tissue and so on.

the anlage that will develop into the kidney.

the intitial stage of mitosis or meiosis in which chromatin condensation begins and the nuclear envelope breaks down.

a very large multi-subunit enzyme complex that degrades ubiquitinated proteins.

the process during which the body plan is laid down along the antero-posterior axis; in vertebrates each spinal segment gives rise to vertebrae and their associated efferent nerves and muscle.

the region around the blastopore lip that provides signals that specify dorsal and ectodermal cell fates during gastrulation.

pairs of identical chromosomes joined at the kinetochore that line up on the metaphase plate of the spindle and separate at anaphase onset.

segmental tissue that will give rise to muscle.

in Drosphila embryos, the first 13 nuclear divisions occur without full cytokinesis within a large syncytial embryo; cellularization occurs only during cycle 14, with the exception of the germ cells. 
Tailbud

Ubiquitin

Ventralizing

YSL (I/E) the region from which the growing tail extends in zebrafish and other teleosts.

a small protein added as a chain to proteins destined for degradation by the ubiquitin conjugating enzymes of the APC/C.

a treatment that enhances ventro-posterior structures in an embryo at the expense of dorsal structures; an extreme example is the complete absence of a nervous system.

the I-YSL (interior yolk syncytial layer) forms the boundary between the blastodisc and the yolk in teleost embryos; the cell cytoplasm of the I-YSL cells is continuous with the yolk; the EYSL (exterior YSL) is a ring of YSL cells beneath the EVL cells that provides the motile force for epiboly, the spreading of the blastodisc over the yolk.

Zygote the one-celled embryo that results from the fusion of sperm and egg and the subsequent fusion of sperm and egg nuclei.

\section{REFERENCES}

1. Aanstad P, Whitaker M. Predictability of dorso-ventral asymmetry in the cleavage stage zebrafish embryo: an analysis using lithium sensitivity as a dorso-ventral marker. Mech Dev. 1999; 88:33-41. [PubMed: 10525186]

2. Aarhus R, Dickey DM, Graeff RM, Gee KR, Walseth TF, Lee HC. Activation and inactivation of $\mathrm{Ca}^{2+}$ release by NAADP ${ }^{+}$J Biol Chem. 1996; 271:8513-8516. [PubMed: 8621471]

3. Aarhus R, Graeff RM, Dickey DM, Walseth TF, Lee HC. ADP-ribosyl cyclase and CD38 catalyze the synthesis of a calcium-mobilizing metabolite from NADP. 1995; 270:30327-30333.

4. Abassi YA, Carroll DJ, Giusti AF, Belton RJ, Foltz KR. Evidence that Src-type tyrosine kinase activity is necessary for initiation of calcium release at fertilization in sea urchin eggs. Dev Biol. 2000; 218:206-219. [PubMed: 10656764]

5. Abassi YA, Foltz KR. Tyrosine phosphorylation of the egg receptor for sperm at fertilization. Dev Biol. 1994; 164:430-443. [PubMed: 8045346]

6. Abou-Haila A, Tulsiani DR. Mammalian sperm acrosome: formation, contents, and function. Arch Biochem Biophys. 2000; 379:173-182. [PubMed: 10898932]

7. Abraham VC, Gupta S, Fluck RA. Ooplasmic segregation in the medaka (Oryzias latipes) egg. Biol Bull. 1993; 184:115-124.

8. Abraham VC, Miller AL, Fluck RA. Microtubule arrays during ooplasmic segregation in the medaka fish egg (Oryzias latipes). Biol Bull. 1995; 188:136-145.

9. Adkins CE, Taylor CW. Lateral inhibition of inositol 1,4,5-trisphosphate receptors by cytosolic $\mathrm{Ca}^{2+}$ Curr Biol. 1999; 9:1115-1118. [PubMed: 10531009]

10. Afzelius BA. A human syndrome caused by immotile cilia. Science. 1976; 193:317-319. [PubMed: 1084576]

11. Aizawa H, Kawahara H, Tanaka K, Yokosawa H. Activation of the proteasome during Xenopus egg activation implies a link between proteasome activation and intracellular calcium release. Biochem Biophys Res Commun. 1996; 218:224-228. [PubMed: 8573136]

12. Akiyama SK. Integrins in cell adhesion and signaling. Hum Cell. 1996; 9:181-186. [PubMed: 9183647]

13. Albrieux M, Lee HC, Villaz M. Calcium signaling by cyclic ADP-ribose, NAADP, and inositol trisphosphate are involved in distinct functions in ascidian oocytes. J Biol Chem. 1998; 273:14566-14574. [PubMed: 9603972] 
14. Albrieux M, Sardet C, Villaz M. The two intracellular $\mathrm{Ca}^{2+}$ release channels, ryanodine receptor and inositol 1,4,5-trisphosphate receptor, play different roles during fertilization in ascidians. Dev Biol. 1997; 189:174-185. [PubMed: 9299112]

15. Allan VJ, Schroer TA. Membrane motors. Curr Opin Cell Biol. 1999; 11:476-482. [PubMed: 10449338]

16. Allbritton NL, Meyer T. Localized calcium spikes and propagating calcium waves. Cell Calcium. 1993; 14:691-697. [PubMed: 8131187]

17. Allen RD, Griffin JL. The time sequence of early events in the fertilization of sea urchin eggs. Exp Cell Res. 1958; 15:163-173. [PubMed: 13574169]

18. Aoto M, Sato K, Takeba S, Horiuchi Y, Iwasaki T, Tokmakov AA, Fukami Y. A 58-kDa Shc protein is present in Xenopus eggs and is phosphorylated on tyrosine residues upon egg activation. Biochem Biophys Res Commun. 1999; 258:265-270. [PubMed: 10329376]

19. Arendt D, Nübler-Jung K. Dorsal or ventral: similarities in fate maps and gastrulation patterns in annelids, arthropods and chordates. Mech Dev. 1997; 61:7-21. [PubMed: 9076674]

20. Ashby MC, Tepikin AV. Polarized calcium and calmodulin signaling in secretory epithelia. Physiol Rev. 2002; 82:701-734. [PubMed: 12087133]

21. Ault KT, Durmowicz G, Galione A, Harger PL, Busa WB. Modulation of Xenopus embryo mesoderm-specific gene expression and dorsoanterior patterning by receptors that activate the phosphatidylinositol cycle signal transduction pathway. Development. 1996; 122:2033-2041. [PubMed: 8681784]

22. Ayabe T, Kopf GS, Schultz RM. Regulation of mouse egg activation: presence of ryanodine receptors and effects of microinjected ryanodine and cyclic ADP ribose on uninseminated and inseminated eggs. Development. 1995; 121:2233-2244. [PubMed: 7635066]

23. Baitinger C, Alderton J, Poenie M, Schulman H, Steinhardt RA. Multifunctional $\mathrm{Ca}^{2+} /$ calmodulindependent protein kinase is necessary for nuclear-envelope breakdown. J Cell Biol. 1990; 111:1763-1773. [PubMed: 2229172]

24. Baker PF, Presley R. A direct method of measuring the rate of entry of sperm into sea urchin eggs. J Physiol. 1966; 186:47p-49p.

25. Baker PF, Presley R. Promotion of polyspermy in the sea-urchin egg by nicotine and its antagonism by curare. J Physiol. 1971; 213:62p-63p.

26. Bandyopadhyay J, Lee J, Lee J, Lee Jin I, Yu J-R, Jee C, Cho J-H, Jung S, Lee Myon H, Zannoni S, Singson A, Kim Do H, Koo H-S, Ahnn J. Calcineurin, a calcium/calmodulin-dependent protein phosphatase, is involved in movement, fertility, egg laying, and growth in Caenorhabditis elegans. Mol Biol Cell. 2002; 13:3281-3293. [PubMed: 12221132]

27. Barber B, Da Cruz MJB, DeLeon J, Fluck RA, Hasenfeld MP, Unis LA. Pacemaker region in the rythmically contracting embryonic epithelium, the enveloping layer of Oryzias latipes, a teleost. J Exp Zool. 1987; 242:35-42. [PubMed: 3598512]

28. Bayer KU, De Koninck P, Schulman H. Alternative splicing modulates the frequency-dependent response of CaMKII to $\mathrm{Ca}^{2+}$ oscillations. EMBO J. 2002; 21:3590-3597. [PubMed: 12110572]

29. Baylis HA, Furuichi T, Yoshikawa F, Mikoshiba K, Satelle DB. Inositol 1,4,5-trisphosphate receptors are strongly expressed in nervous system, pharynx, intestine, gonad and excretory cell of Caenorhabditis elegans and are encoded by a single gene (itr-1). J Mol Biol. 1999; 294:467-476. [PubMed: 10610772]

30. Becchetti A, Whitaker M. Lithium blocks cell cycle transitions in the first cell cycles of sea urchin embryos, an effect rescued by myo-inositol. Development. 1997; 124:1099-1107. [PubMed: 9102297]

31. Begg DA, Rebhun LI. pH regulates the polymerization of actin in the sea urchin egg cortex. J Cell Biol. 1979; 83:241-248. [PubMed: 41844]

32. Bement WM, Capco DG. Activators of protein kinase C trigger cortical granule exocytosis, cortical contraction, and cleavage furrow formation in Xenopus laevis oocytes and eggs. J Cell Biol. 1989; 108:885-892. [PubMed: 2493460]

33. Ben-Yosef D, Shalgi R. Early ionic events in activation of the mammalian egg. Rev Reprod. 1998; 3:96-103. [PubMed: 9685188] 
34. Berridge G, Dickinson G, Parrington J, Galione A, Patel S, et al. Solubilization of receptors for the novel $\mathrm{Ca}^{2+}$-mobilizing messenger, nicotinic acid adenine dinucleotide phosphate. J Biol Chem. 2002; 277:43717-43723. [PubMed: 12223470]

35. Berridge MJ. Inositol trisphosphate and calcium mobilization. Ciba Found Symp. 1986; 122:39_ 49. [PubMed: 3491745]

36. Berridge MJ. Inositol trisphosphate and calcium signalling. Nature. 1993; 361:315-325. [PubMed: 8381210]

37. Berridge MJ. Phosphatidylinositol hydrolysis and calcium signaling. Advances in cyclic nucleotide research. 1981; 14:289-299. [PubMed: 6269383]

38. Berridge MJ, Downes CP, Hanley MR. Lithium amplifies agonist-dependent phosphatidylinositol responses in brain and salivary glands. Biochem J. 1982; 206:587-595. [PubMed: 7150264]

39. Berridge MJ, Downes CP, Hanley MR. Neural and developmental actions of lithium: a unifying hypothesis. Cell. 1989; 59:411-419. [PubMed: 2553271]

40. Berridge MJ, Galione A. Cytosolic calcium oscillators. FASEB J. 1988; 2:3074-3082. [PubMed: 2847949]

41. Berridge MJ, Lipp P, Bootman MD. The versatility and universality of calcium signalling. Nat Rev Mol Cell Biol. 2000; 1:11-21. [PubMed: 11413485]

42. Berrie CP, Cuthbertson KS, Parrington J, Lai FA, Swann K. A cytosolic sperm factor triggers calcium oscillations in rat hepatocytes. Biochem J. 1996; 313:369-372. [PubMed: 8573066]

43. Bi GQ, Alderton JM, Steinhardt RA. Calcium-regulated exocytosis is required for cell membrane resealing. J Cell Biol. 1995; 131:1747-1758. [PubMed: 8557742]

44. Bi GQ, Morris RL, Liao G, Alderton JM, Scholey JM, Steinhardt RA. Kinesin- and myosin-driven steps of vesicle recruitment for $\mathrm{Ca}^{2+}$ regulated exocytosis. J Cell Biol. 1997; 138:999-1008. [PubMed: 9281579]

45. Billington RA, Ho A, Genazzani AA. Nicotinic acid adenine dinucleotide phosphate (NAADP) is present at micromolar concentrations in sea urchin spermatozoa. J Physiol. 2002; 544:107-112. [PubMed: 12356884]

46. Bird JM, Houghton JA. The activation of mammalian sperm. Sci Prog. 1991; 75:107-120. [PubMed: 1792553]

47. Bloom TL, Szuts EZ, Eckberg WR. Inositol trisphosphate, inositol phospholipid metabolism, and germinal vesicle breakdown in surf clam oocytes. Dev Biol. 1988; 129:532-540. [PubMed: 2843404]

48. Borodinsky LN, Root CM, Cronin JA, Sann SB, Gu X, Spitzer NC. Activity-dependent homeostatic specification of transmitter expression in embryonic neurons. Nature. 2004; 429:523530. [PubMed: 15175743]

49. Brady RC, Hilfer SR. Optic cup formation: a calcium regulated process. Proc Natl Acad Sci U S A. 1982; 79:5587-5591. [PubMed: 6813862]

50. Brandriff B, Hinegardner RI, Steinhardt R. Development and life cycle of the parthenogenetically activated sea urchin embryo. J Exp Zool. 1975; 192:13-24. [PubMed: 1092807]

51. Brind S, Swann K, Carroll J. Inositol 1,4,5-trisphosphate receptors are downregulated in mouse oocytes in response to sperm or adenophostin $\mathrm{A}$ but not to increases in intracellular $\mathrm{Ca}^{2+}$ or egg activation. Dev Biol. 2000; 223:251-265. [PubMed: 10882514]

52. Brooker G, Seki T, Croll D, Wahlestedt C. Calcium wave evoked by activation of endogenous or exogenously expressed receptors in Xenopus oocytes. Proc Natl Acad Sci U S A. 1990; 87:28132817. [PubMed: 2157216]

53. Brownlee C, Dale B. Temporal and spatial correlation of fertilization current, calcium waves and cytoplasmic contraction in eggs of Ciona intestinalis. Proc R Soc Lond B Biol Sci. 1990; 239:321328. [PubMed: 1972794]

54. Busa WB, Gimlich RL. Lithium-induced teratogenesis in frog embryos prevented by a polyphosphoinositide cycle intermediate or a diacylglycerol analog. Dev Biol. 1989; 132:315-324. [PubMed: 2538373]

55. Busa WB, Nuccitelli R. An elevated free cytosolic $\mathrm{Ca}^{2+}$ wave follows fertilization in eggs of the frog, Xenopus laevis. J Cell Biol. 1985; 100:1325-1329. [PubMed: 3980584] 
56. Campanella C, Talevi R, Kline D, Nuccitelli R. The cortical reaction in the egg of Discoglossus pictus: a study of the changes in the endoplasmic reticulum at activation. Dev Biol. 1988; 130:108-119. [PubMed: 3181623]

57. Campbell KD, Reed WA, White KL. Ability of integrins to mediate fertilization, intracellular calcium release, and parthenogenetic development in bovine oocytes. Biol Reprod. 2000; 62:1702-1709. [PubMed: 10819774]

58. Carrasco D, Allende CC, Allende JE. The incorporation of myo-inositol into phosphatidylinositol derivatives is stimulated during hormone-induced meiotic maturation. Exp Cell Res. 1990; 191:313-318. [PubMed: 2257883]

59. Carroll AG, Eckberg WR. Possible involvement of calmodulin in maturation and activation of Chaetopterus eggs. Dev Biol. 1983; 99:1-6. [PubMed: 6617994]

60. Carroll DJ, Albay DT, Terasaki M, Jaffe LA, Foltz KR. Identification of PLC $\gamma$-dependent and independent events during fertilization of sea urchin eggs. Dev Biol. 1999; 206:232-247. [PubMed: 9986735]

61. Carroll DJ, Ramarao CS, Mehlmann LM, Roche S, Terasaki M, Jaffe LA. Calcium release at fertilization in starfish eggs is mediated by phospholipase C $\gamma$. J Cell Biol. 1997; 138:1303-1311. [PubMed: 9298985]

62. Carroll EJ, Endress AG. Sea urchin fertilization envelope: uncoupling of cortical granule exocytosis from envelope assembly and isolation of an envelope intermediate from Strongylocentrotus purpuratus embryos. Dev Biol. 1982; 94:252-258. [PubMed: 7152106]

63. Carroll J, Swann K. Spontaneous cytosolic calcium oscillations driven by inositol trisphosphate occur during in vitro maturation of mouse oocytes. J Biol Chem. 1992; 267:11196-11201. [PubMed: 1597455]

64. Carroll J, Swann K, Whittingham D, Whitaker M. Spatiotemporal dynamics of intracellular $\left[\mathrm{Ca}^{2+}\right]_{\mathrm{i}}$ oscillations during the growth and meiotic maturation of mouse oocytes. Development. 1994; 120:3507-3517. [PubMed: 7821218]

65. Carroll M, Levasseur M, Wood C, Whitaker MJ, Jones KT, McDougall A. Exploring the mechanism of action of the sperm-triggered calcium-wave pacemaker in ascidian zygotes. $\mathrm{J}$ Cell Sci. 2003; 116:4997-5004. [PubMed: 14625393]

66. Casazza G, De Santis R, Pinto MR. Sperm binding to eggs of Ciona intestinalis. Role of $\mathrm{Ca}^{2+}$ experimental cell research. Exp Cell Res. 1984; 155:261-266. [PubMed: 6436043]

67. Centonze, VE.; White, JG. Calcium oscillations during early C. elegans development. 1995. http:// elegansswmededu/wli/[wm95e148] (cited with permission)

68. Chambers EL. Fertilization and cleavage of the eggs of the sea urchin Lytechinus variegatus in $\mathrm{Ca}^{2+}$-free sea water. Eur J Cell Biol. 1980; 22:476.

69. Chambers EL, Angeloni SV. Is external calcium required for fertilization of sea urchin eggs by acrosome-reacted sperm? J Cell Biol. 1981; 91:181a.

70. Chambers EL, de Armendi J. Membrane potential, action potential and activation potential of eggs of the sea urchin, Lytechinus variegatus. Exp Cell Res. 1979; 122:203-218. [PubMed: 582584]

71. Chambers EL, McCulloh DH. Excitation, activation and sperm entry in voltage-clamped sea urchin eggs. J Reprod Fertil. 1990:117-132. [PubMed: 2374113]

72. Chambers EL, Pressman BC, Rose B. The activation of sea urchin eggs by the divalent ionophores A23187 and X-537A. Biochem Biophys Res Commun. 1974; 60:126-132. [PubMed: 4472499]

73. Chang DC, Lu P. Multiple types of calcium signals are associated with cell division in zebrafish embryo. Microsc Res Tech. 2000; 49:111-122. [PubMed: 10816249]

74. Chang DC, Meng CL. A localized elevation of cytosolic-free calcium is associated with cytokinesis in the zebrafish embryo. J Cell Biol. 1995; 131:1539-1545. [PubMed: 8522610]

75. Charbonneau M, Moreau M, Picheral B, Vilain JP, Guerrier P. Fertilization of amphibian eggs: a comparison of electrical responses between anurans and urodeles. Dev Biol. 1983; 98:304-318. [PubMed: 6603379]

76. Chen RH, Murray A. Characterization of spindle assembly checkpoint in Xenopus egg extracts. Methods Enzymol. 1997

77. Cheng H, Lederer WJ, Cannell MB. Calcium sparks: elementary events underlying excitationcontraction coupling in heart muscle. Science. 1993; 262:740-744. [PubMed: 8235594] 
78. Chernoff EAG, Hilfer SR. Calcium dependence and contraction in somite formation. Tissue Cell. 1982; 14:435-449. [PubMed: 6815826]

79. Cheung A, Swann K, Carroll J. The ability to generate normal $\mathrm{Ca}^{2+}$ transients in response to spermatozoa develops during the final stages of oocyte growth and maturation. Hum Reprod. 2000; 15:1389-1395. [PubMed: 10831575]

80. Chiba K, Kado RT, Jaffe LA. Development of calcium release mechanisms during starfish oocyte maturation. Dev Biol. 1990; 140:300-306. [PubMed: 2373255]

81. Chini EN, Beers KW, Dousa TP. Nicotinate adenine dinucleotide phosphate (NAADP) triggers a specific calcium release system in sea urchin eggs. J Biol Chem. 1995; 270:3216-3223. [PubMed: 7852407]

82. Chini EN, De Toledo FGS. Nicotinic acid adenine dinucleotide phosphate: a new intracellular second messenger? Am J Physiol. 2002; 282:C1191-1198.

83. Cho C, Bunch DO, Faure JE, Goulding EH, Eddy EM, Primakoff P, Myles DG. Fertilization defects in sperm from mice lacking fertilin beta. Science. 1998; 281:1857-1859. [PubMed: 9743500]

84. Choi S-C, Han J-K. Xenopus Cdc42 regulates convergent extension movements during gastrulation through Wnt/Ca2+ signaling pathway. Dev Biol. 2002; 244:342-357. [PubMed: 11944942]

85. Churchill GC, Galione A. Spatial control of $\mathrm{Ca}^{2+}$ signaling by nicotinic acid adenine dinucleotide phosphate diffusion and gradients. J Biol Chem. 2000; 275:38687-38692. [PubMed: 11006280]

86. Churchill GC, O’Neill JS, Masgrau R, Patel S, Thomas JM, Genazzani AA, Galione A. Sperm deliver a new second messenger: NAADP. Curr Biol. 2003; 13:125-128. [PubMed: 12546785]

87. Churchill Grant C, Okada Y, Justyn M Thomas, Armando A Genazzani, Patel S, Galione A. NAADP mobilizes $\mathrm{Ca}(2+)$ from reserve granules, lysosome-related organelles, in sea urchin eggs. Cell. 2002; 111:703-708. [PubMed: 12464181]

88. Ciapa B, Borg B, Whitaker M. Polyphosphoinositide metabolism during the fertilization wave in sea urchin eggs. Development. 1992; 115:187-195. [PubMed: 1322270]

89. Ciapa B, Chiri S. Egg activation: upstream of the fertilization calcium signal. Biol Cell. 2000; 92:215-233. [PubMed: 11043410]

90. Ciapa B, Epel D. An early increase in cGMP follows fertilization of sea urchin eggs. Biochem Biophys Res Commun. 1996; 223:633-636. [PubMed: 8687447]

91. Ciapa B, Epel D. A rapid change in phosphorylation on tyrosine accompanies fertilization of sea urchin eggs. FEBS Lett. 1991; 295:167-170. [PubMed: 1722461]

92. Ciapa B, Maggio K. Effect of lithium on ionic balance and polyphosphoinositide metabolism during larval vegetalization of the sea urchin Paracentrotus lividus. Dev Biol. 1993; 159:114-121. [PubMed: 8396053]

93. Ciapa B, Pesando D, Wilding M, Whitaker M. Cell-cycle calcium transients driven by cyclic changes in inositol trisphosphate levels. Nature. 1994; 368:875-878. [PubMed: 8159248]

94. Ciapa B, Whitaker M. Two phases of inositol polyphosphate and diacylglycerol production at fertilisation. FEBS Lett. 1986; 195:347-351. [PubMed: 3080333]

95. Clandinin TR, DeModena JA, Sternberg PW. Inositol trisphosphate mediates a RAS-independent response to LET-23 receptor kinase activation in C. elegans. Cell. 1998; 92:523-533. [PubMed: 9491893]

96. Clapham DE. Calcium signaling. Cell. 1995; 80:259-268. [PubMed: 7834745]

97. Clapper DL, Walseth TF, Dargie PJ, Lee HC. Pyridine nucleotide metabolites stimulate calcium release from sea urchin egg microsomes desensitized to inositol trisphosphate. J Biol Chem. 1987; 262:9561-9568. [PubMed: 3496336]

98. Colas P, Dube F. Meiotic maturation in mollusc oocytes. Semin Cell Dev Biol. 1998; 9:539-548. [PubMed: 9835642]

99. Coward K, Campos-Mendoza A, Larman M, Hibbitt O, McAndrew B, Bromage N, Parrington J. Teleost fish spermatozoa contain a cytosolic protein factor that induces calcium release in sea urchin egg homogenates and triggers calcium oscillations when injected into mouse oocytes. Biochem Biophys Res Commun. 2003; 305:299-304. [PubMed: 12745073] 
100. Cox LJ, Larman MG, Saunders CM, Hashimoto K, Swann K, Lai FA. Sperm phospholipase CS from humans and cynomolgus monkeys triggers $\mathrm{Ca} 2+$ oscillations, activation and development of mouse oocytes. Reproduction. 2002; 124:611-623. [PubMed: 12416999]

101. Cran DG, Esper CR. Cortical granules and the cortical reaction in mammals. J Reprod Fertil Suppl. 1990; 42:177-188. [PubMed: 2077122]

102. Crèton R, Kreiling JA, Jaffe LF. Presence and roles of calcium gradients along the dorso-ventral axis in Drosophila embryos. Dev Biol. 2000; 217:375-385. [PubMed: 10625561]

103. Crèton R, Speksnijder JE, Jaffe LF. Patterns of free calcium in zebrafish embryos. J Cell Sci. 1998; 111:1613-1622. [PubMed: 9601092]

104. Crossley I, Swann K, Chambers E, Whitaker M. Activation of sea urchin eggs by inositol phosphates is independent of external calcium. Biochem J. 1988; 252:257-262. [PubMed: 3421904]

105. Crossley I, Whalley T, Whitaker M. Guanosine $5^{\prime}$-thiotriphosphate may stimulate phosphoinositide messenger production in sea urchin eggs by a different route than the fertilizing sperm. Cell Regul. 1991; 2:121-133. [PubMed: 1650582]

106. Csordas G, Thomas AP, Hajnoczky G. Calcium signal transmission between ryanodine receptors and mitochondria in cardiac muscle. Trends Cardiovasc Med. 2001; 11:269-275. [PubMed: 11709280]

107. Dale B, De Felice LJ, Taglietti V. Membrane noise and conductance increase during single spermatozoon-egg interactions. Nature. 1978; 275:217-219. [PubMed: 29229]

108. Dale B, Fortunato A, Monfrecola V, Tosti E. A soluble sperm factor gates $\mathrm{Ca}^{2+}$-activated $\mathrm{K}^{+}$ channels in human oocytes. J Assist Reprod Genet. 1996; 13:573-577. [PubMed: 8844315]

109. Damsky C, Sutherland A, Fisher S. Extracellular matrix 5: adhesive interactions in early mammalian embryogenesis, implantation, and placentation. FASEB J. 1993; 7:1320-1329. [PubMed: 8224605]

110. Darszon A, Labarca P, Nishigaki T, Espinosa F. Ion channels in sperm physiology. Physiol Rev. 1999; 79:481-510. [PubMed: 10221988]

111. David C, Halliwell J, Whitaker M. Some properties of the membrane currents underlying the fertilization potential in sea urchin eggs. J Physiol. 1988; 402:139-154. [PubMed: 2466981]

112. Davidson EH, Rast JP, Oliveri P, Ransick A, Calestani C, Yuh C-H, Minokawa T, Amore G, Hinman V, Arenas-Mena C, Otim O, Brown CT, Livi CB, Lee PY, Revilla R, Rust AG, Pan Zj, Schilstra MJ, Clarke PJC, Arnone MI, Rowen L, Cameron RA, McClay DR, Hood L, Bolouri H. A genomic regulatory network for development. Science. 2002; 295:1669-1678. [PubMed: 11872831]

113. Day ML, McGuinness OM, Berridge MJ, Johnson MH. Regulation of fertilization-induced $\mathrm{Ca}^{2+}$ spiking in the mouse zygote. Cell Calcium. 2000; 28:47-54. [PubMed: 10942703]

114. Deguchi R, Osanai K. Meiosis reinitiation from the first prophase is dependent on the levels of intracellular $\mathrm{Ca}^{2+}$ and $\mathrm{pH}$ in oocytes of the bivalves Mactra chinensis and Limaria hakodatensis. Dev Biol. 1994; 166:587-599. [PubMed: 7813778]

115. Deguchi R, Osanai K, Morisawa M. Extracellular $\mathrm{Ca}^{2+}$ entry and $\mathrm{Ca}^{2+}$ release from inositol 1,4,5-trisphosphate-sensitive stores function at fertilization in oocytes of the marine bivalve Mytilus edulis. Development. 1996; 122:3651-3660. [PubMed: 8951080]

116. Deguchi R, Shirakawa H, Oda S, Mohri T, Miyazaki S. Spatiotemporal analysis of $\mathrm{Ca}^{2+}$ waves in relation to the sperm entry site and animal-vegetal axis during $\mathrm{Ca}^{2+}$ oscillations in fertilized mouse eggs. Dev Biol. 2000; 218:299-313. [PubMed: 10656771]

117. Deng MQ, Huang XY, Tang TS, Sun FZ. Spontaneous and fertilization-induced $\mathrm{Ca}^{2+}$ oscillations in mouse immature germinal vesicle-stage oocytes. Biol Reprod. 1998; 58:807-813. [PubMed: 9510970]

118. Denton RM, McCormack JG, Midgley PJ, Rutter GA, Thomas AP. The role of $\mathrm{Ca}^{2+}$ in the hormonal control of intramitochondrial metabolism in heart, liver, and adipose tissue. Adv Second Messenger Phosphoprotein Res. 1988

119. Diaz J, Baier G, Martinez-Mekler G, Pastor N. Interaction of the $\mathrm{IP}_{3}-\mathrm{Ca}^{2+}$ and the FGF-MAPK signaling pathways in the Xenopus laevis embryo: a qualitative approach to the mesodermal induction problem. Biophys Chem. 2002; 97:55-72. [PubMed: 12052495] 
120. Dong JB, Tang TS, Sun FZ. Xenopus and chicken sperm contain a cytosolic soluble protein factor which can trigger calcium oscillations in mouse eggs. Biochem Biophys Res Commun. 2000; 268:947-951. [PubMed: 10679311]

121. Dousa TP, Chini EN, Beers KW. Adenine nucleotide diphosphates: emerging second messengers acting via intracellular $\mathrm{Ca}^{2+}$ release. Am J Physiol. 1996; 271:C1007-1024. [PubMed: 8897805]

122. Dréan G, Leclerc C, Duprat AM, Moreau M. Expression of L-type Ca2+ channel during early embryogenesis in Xenopus laevis. Int J Dev Biol. 1995; 39:1027-1032. [PubMed: 8901206]

123. Du S, Purcell S, Christian J, McGrew L, Moon R. Identification of distinct classes and functional domains of Wnts through expression of wild-type and chimeric proteins in Xenopus embryos. Mol Cell Biol. 1995; 15:2625-2634. [PubMed: 7739543]

124. Dube F. The relationships between early ionic events, the pattern of protein synthesis, and oocyte activation in the surf clam, Spisula solidissima. Dev Biol. 1988; 126:233-241. [PubMed: 3350208]

125. Dube F, Guerrier P. Activation of Barnea candida (Mollusca, Pelecypoda) oocytes by sperm of $\mathrm{KCl}$, but not by $\mathrm{NH}_{4} \mathrm{Cl}$, requires a calcium influx. Dev Biol. 1982; 92:408-417. [PubMed: 7117692]

126. Ducibella T, Huneau D, Angelichio E, Xu Z, Richard M Schultz, Gregory S Kopf, Fissore R, Madoux S, Ozil J-P, et al. Egg-to-embryo transition is driven by differential responses to $\mathrm{Ca}^{2+}$ oscillation number. Dev Biol. 2002; 250:280-291. [PubMed: 12376103]

127. Dumollard R, Carroll J, Dupont G, Sardet C. Calcium wave pacemakers in eggs. J Cell Sci. 2002; 115:3557-3564. [PubMed: 12186942]

128. Dumollard R, Hammar K, Porterfield M, Smith PJ, Cibert C, Rouviere C, Sardet C. Mitochondrial respiration and $\mathrm{Ca}^{2+}$ waves are linked during fertilization and meiosis completion. Development. 2003; 130:683-692. [PubMed: 12505999]

129. Dumollard R, Marangos P, Fitzharris G, Swann K, Duchen M, Carroll J. Sperm-triggered [Ca ${ }^{2+}$ ] oscillations and $\mathrm{Ca}^{2+}$ homeostasis in the mouse egg have an absolute requirement for mitochondrial ATP production. Development. 2004; 131:3057-3067. [PubMed: 15163630]

130. Dumollard R, Sardet C. Three different calcium wave pacemakers in ascidian eggs. J Cell Sci. 2001; 114:2471-2481. [PubMed: 11559755]

131. Dupont G, McGuinness OM, Johnson MH, Berridge MJ, Borgese F. Phospholipase C in mouse oocytes: characterization of $\beta$ and $\gamma$ isoforms and their possible involvement in sperm-induced $\mathrm{Ca}^{2+}$ spiking. Biochem J. 1996; 316:583-591. [PubMed: 8687404]

132. Ehrlich BE. Functional properties of intracellular calcium-release channels. Curr Opin Neurobiol. 1995; 5:304-309. [PubMed: 7580152]

133. Eisen A, Kiehart DP, Wieland SJ, Reynolds GT. Temporal sequence and spatial distribution of early events of fertilization in single sea urchin eggs. J Cell Biol. 1984; 99:1647-1654. [PubMed: 6490715]

134. Eisen A, Reynolds GT. Calcium transients during early development in single starfish (Asterias forbesi) oocytes. J Cell Biol. 1984; 99:1878-1882. [PubMed: 6490725]

135. Eisen A, Reynolds GT. Source and sinks for the calcium released during fertilization of single sea urchin eggs. J Cell Biol. 1985; 100:1522-1527. [PubMed: 3988799]

136. Elul T, Keller R. Monopolar protrusive activity: a new morphogenetic cell behaviour in the neural plate dependent on vertical interactions with the mesoderm in Xenopus. Dev Biol. 2000:3-19. [PubMed: 10898957]

137. Endo M, Tanaka M, Ogawa Y. Calcium induced release of calcium from the sarcoplasmic reticulum of skinned skeletal muscle fibres. Nature. 1970; 228:34-36. [PubMed: 5456208]

138. Epel D. The initiation of development at fertilization. Cell Differ Dev. 1990; 29:1-12. [PubMed: 2154300]

139. Epel D, Perry G, Schmidt T. Intracellular calcium and fertilization: role of the cation and regulation of intracellular calcium levels. Prog Clin Biol Res. 1982; 91:171-183. [PubMed: 6292942]

140. Epel D, Schatten G. Daniel Mazia: a passion for understanding how cells reproduce. Trends Cell Biol. 1998; 8:416-418. [PubMed: 9789331] 
141. Epel D, Steinhardt R, Humphreys T, Mazia D. An analysis of the partial metabolic derepression of sea urchin eggs by ammonia: the existence of independent pathways. Dev Biol. 1974; 40:245255. [PubMed: 4609819]

142. Essner JJ, Vogan KJ, Wagner MK, Tabin CJ, Yost HJ, Brueckner M. Conserved function for embryonic nodal cilia. Nature. 2002; 418:37-38. [PubMed: 12097899]

143. Eto K, Puzon-McLaughlin W, Sheppard D, Sehara-Fujisawa A, Zhang XP, Takada Y. RGDindependent binding of integrin a $9 \beta 1$ to the ADAM-12 and - 15 disintegrin domains mediates cell-cell interaction. J Biol Chem. 2000; 275:34922-34930. [PubMed: 10944520]

144. Evans JP, Kopf GS, Schultz RM. Characterization of the binding of recombinant mouse sperm fertilin beta subunit to mouse eggs: evidence for adhesive activity via an egg beta1 integrinmediated interaction. Dev Biol. 1997; 187:79-93. [PubMed: 9224676]

145. Evans T, Rosenthal ET, Youngblom J, Distel D, Hunt T. Cyclin: a protein specified by maternal mRNA in sea urchin eggs that is destroyed at each cleavage division. Cell. 1983; 33:389-396. [PubMed: 6134587]

146. Fenichel P, Durand-Clement M. Role of integrins during fertilization in mammals. Hum Reprod. 1998; 13:31-46. [PubMed: 10091056]

147. Fernandez-Busquets X, Burger MM. Cell adhesion and histocompatibility in sponges. Microsc Res Tech. 1999; 44:204-218. [PubMed: 10098923]

148. Ferrari MB, Rohrbough J, Spitzer NC. Spontaneous calcium transients regulate myofibrillogenesis in embryonic Xenopus myocytes. Dev Biol. 1996; 178:484-497. [PubMed: 8812144]

149. Ferreira MC, Hilfer SR. Calcium regulation of neural fold formation: visualization of the actin cytoskeleton in living chick embryos. Dev Biol. 1993; 159:427-440. [PubMed: 8405669]

150. Fissore RA, Pinto-Correia C, Robl JM. Inositol trisphosphate-induced calcium release in the generation of calcium oscillations in bovine eggs. Biol Reprod. 1995; 53:766-774. [PubMed: 8547468]

151. Fissore RA, Robl JM. Mechanism of calcium oscillations in fertilized rabbit eggs. Dev Biol. 1994; 166:634-642. [PubMed: 7813782]

152. Fissore RA, Robl JM. Sperm, inositol trisphosphate, and thimerosal-induced intracellular $\mathrm{Ca}^{2+}$ elevations in rabbit eggs. Dev Biol. 1993; 159:122-130. [PubMed: 8365556]

153. FitzHarris G, Marangos P, Carroll J. Cell cycle-dependent regulation of the structure of the endoplasmic reticulum and inositol 1,4,5-trsphosphate-induced $\mathrm{Ca}^{2+}$ release in mouse oocytes and embryos. Mol Biol Cell. 2003; 14:288-301. [PubMed: 12529444]

154. Fluck R, Abraham V, Miller A, Galione A. Microinjection of cyclic ADP-ribose triggers a regenerative wave of $\mathrm{Ca}^{2+}$ release and exocytosis of cortical alveoli in medaka eggs. Zygote. 1999; 7:285-292. [PubMed: 10717946]

155. Fluck RA, Miller AL, Jaffe LF. Calcium waves accompany contraction waves in the Oryzias latipes (medaka) blastoderm. Biol Bull. 1991; 181:352.

156. Fluck RA, Miller AL, Jaffe LF. Slow calcium waves accompany cytokinesis in medaka fish eggs. J Cell Biol. 1991; 115:1259-1265. [PubMed: 1955473]

157. Fontanilla RA, Nuccitelli R. Characterization of the sperm-induced calcium wave in Xenopus eggs using confocal microscopy. Biophys J. 1998; 75:2079-2087. [PubMed: 9746550]

158. Fujiwara T, Nakada K, Shirakawa H, Miyazaki S. Development of inositol trisphosphate-induced calcium release mechanism during maturation of hamster oocytes. Dev Biol. 1993; 156:69-79. [PubMed: 8383620]

159. Galione A. Cyclic ADP-ribose, the ADP-ribosyl cyclase pathway and calcium signalling. Mol Cell Endocrinol. 1994; 98:125-131. [PubMed: 8143921]

160. Galione A, Churchill GC. Cyclic ADP ribose as a calcium-mobilizing messenger. Sci STKE. 2000; 2000:Pe1. [PubMed: 11752598]

161. Galione A, Cui Y, Empson R, Iino S, Wilson H, Terrar D. Cyclic ADP-ribose and the regulation of calcium-induced calcium release in eggs and cardiac myocytes. Cell Biochem Biophys. 1998; 28:19-30. [PubMed: 9386890] 
162. Galione A, Jones KT, Lai FA, Swann K. A cytosolic sperm protein factor mobilizes $\mathrm{Ca}^{2+}$ from intracellular stores by activating multiple $\mathrm{Ca}^{2+}$ release mechanisms independently of low molecular weight messengers. J Biol Chem. 1997; 272:28901-28905. [PubMed: 9360959]

163. Galione A, Lee HC, Busa WB. $\mathrm{Ca}^{2+}$-induced $\mathrm{Ca}^{2+}$ release in sea urchin egg homogenates: modulation by cyclic ADP-ribose. Science. 1991; 253:1143-1146. [PubMed: 1909457]

164. Galione A, McDougall A, Busa WB, Willmott N, Gillot I, Whitaker M. Redundant mechanisms of calcium-induced calcium release underlying calcium waves during fertilization of sea urchin eggs. Science. 1993; 261:348-352. [PubMed: 8392748]

165. Galione A, Patel S, Churchill GC. NAADP-induced calcium release in sea urchin eggs. Biol Cell. 2000; 92:197-204. [PubMed: 11043408]

166. Galione A, Swann K, Georgiou P, Whitaker M. Regenerative and non-regenerative calcium transients in hamster eggs triggered by inositol 1,4,5-trisphosphate. J Physiol. 1994; 480:465474. [PubMed: 7869260]

167. Geng L, Segal Y, Peissel B, Deng N, Pei Y, Carone F, Rennke HG, Glucksmann-Kuis AM, Schneider MC, Ericsson M, Reeders ST, Zhou J. Identification and localization of polycystin, the PKD1 gene product. J Clin Invest. 1996; 98:2674-2682. [PubMed: 8981910]

168. Gerasimenko JV, Maruyama Y, Yano K, Dolman NJ, Tepikin A, V. Petersen OH, Gerasimenko $\mathrm{O}, \mathrm{V}$. NAADP mobilizes $\mathrm{Ca}^{2+}$ from a thapsigargin-sensitive store in the nuclear envelope by activating ryanodine receptors. J Cell Biol. 2003; 163:271-282. [PubMed: 14568993]

169. Gerasimenko OV, Gerasimenko JV, Tepikin AV, Petersen OH. ATP-dependent accumulation and inositol trisphosphate- or cyclic ADP-ribose-mediated release of $\mathrm{Ca} 2+$ from the nuclear envelope. Cell. 1995; 80:439-444. [PubMed: 7859285]

170. Gilkey JC, Jaffe LF, Ridgway EB, Reynolds GT. A free calcium wave traverses the activating egg of the medaka, Oryzias latipes. J Cell Biol. 1978; 76:448-466. [PubMed: 10605450]

171. Gilland E, Baker R, Denk W. Long duration three-dimensional imaging of calcium waves in zebrafish using multiphoton fluorescence microscopy. Biol Bull. 2003; 205:176-177. [PubMed: 14583515]

172. Gilland E, Miller AL, Karplus E, Baker R, Webb SE. Imaging of multicellular large scale rhythmic calcium waves during zebrafish gastrulation. Proc Natl Acad Sci U S A. 1999; 96:157161. [PubMed: 9874788]

173. Gillot I, Whitaker M. Calcium signals in and around the nucleus in sea urchin eggs. Cell Calcium. 1994; 16:269-278. [PubMed: 7820846]

174. Giusti AF, Carroll DJ, Abassi YA, Terasaki M, Foltz KR, Jaffe LA. Requirement of a Src family kinase for initiating calcium release at fertilization in starfish eggs. J Biol Chem. 1999; 274:29318-29322. [PubMed: 10506191]

175. Giusti AF, O’Neill FJ, Yamasu K, Foltz KR, Jaffe LA. Function of a sea urchin egg Src family kinase in initiating $\mathrm{Ca}^{2+}$ release at fertilization. Dev Biol. 2003; 256:367-378. [PubMed: 12679109]

176. Giusti AF, Xu W, Hinkle B, Terasaki M, Jaffe LA. Evidence that fertilization activates starfish eggs by sequential activation of a Src-like kinase and phospholipase $\mathrm{C} \gamma$. J Biol Chem. 2000; 275:16788-16794. [PubMed: 10747984]

177. Glabe CG. Interaction of the sperm adhesive protein, bindin, with phospholipid vesicles. II. Bindin induces the fusion of mixed-phase vesicles that contain phosphatidylcholine and phosphatidylserine in vitro. J Cell Biol. 1985; 100:800-806. [PubMed: 3972896]

178. Glahn D, Mark SD, Behr RK, Nuccitelli R. Tyrosine kinase inhibitors block sperm-induced egg activation in Xenopus laevis. Dev Biol. 1999; 205:171-180. [PubMed: 9882505]

179. Gordo AC, Kurokawa M, Wu H, Fissore RA. Modifications of the $\mathrm{Ca}^{2+}$ release mechanisms of mouse oocytes by fertilization and by sperm factor. Mol Hum Reprod. 2002; 8:619-629. [PubMed: 12087076]

180. Gordo AC, Rodrigues P, Kurokawa M, Jellerette T, Exley GE, Warner C, Fissore R. Intracellular calcium oscillations signal apoptosis rather than activation in in vitro aged mouse eggs. Biol Reprod. 2002; 66:1828-1837. [PubMed: 12021069] 
181. Gordo AC, Wu H, He CL, Fissore RA. Injection of sperm cytosolic factor into mouse metaphase II oocytes induces different developmental fates according to the frequency of $\left[\mathrm{Ca}^{2+}\right]_{\mathrm{i}}$ oscillations and oocyte age. Biol Reprod. 2000; 62:1370-1379. [PubMed: 10775189]

182. Goud PT, Goud AP, Van Oostveldt P, Dhont M. Presence and dynamic redistribution of type I inositol 1,4,5-trisphosphate receptors in human oocytes and embryos during in-vitro maturation, fertilization and early cleavage divisions. Mol Hum Reprod. 1999; 5:441-451. [PubMed: 10338367]

183. Goudeau H, Depresle Y, Rosa A, Goudeau M. Evidence by a voltage clamp study of an electrically mediated block to polyspermy in the egg of the ascidian Phallusia mammillata. Dev Biol. 1994; 166:489-501. [PubMed: 7813772]

184. Goudeau H, Goudeau M, Guibourt N. The fertilization potential and associated membrane potential oscillations during the resumption of meiosis in the egg of the ascidian Phallusia mammillata. Dev Biol. 1992; 153:227-241. [PubMed: 1397680]

185. Goudeau M, Goudeau H, Guillaumin D. Extracellular $\mathrm{Mg}^{2+}$ induces a loss of microvilli, membrane retrieval, and the subsequent cortical reaction, in the oocyte of the prawn Palaemon serratus. Dev Biol. 1991; 148:31-50. [PubMed: 1936567]

186. Grainger JL, Winkler MM, Shen SS, Steinhardt RA. Intracellular $\mathrm{pH}$ controls protein synthesis rate in the sea urchin egg and early embryo. Dev Biol. 1979; 68:396-406. [PubMed: 35433]

187. Grandin N, Charbonneau M. Changes in intracellular free calcium activity in Xenopus eggs following imposed intracellular $\mathrm{pH}$ changes using weak acids and weak bases. Biochim Biophys Acta. 1991; 1091:242-250. [PubMed: 1704800]

188. Groigno L, Whitaker M. An anaphase calcium signal controls chromosome disjunction in early sea urchin embryos. Cell. 1998; 92:193-204. [PubMed: 9458044]

189. Grumetto L, Wilding M, De Simone ML, Tosti E, Galione A, Dale B. Nitric oxide gates fertilization channels in ascidian oocytes through nicotinamide nucleotide metabolism. Biochem Biophys Res Commun. 1997; 239:723-728. [PubMed: 9367836]

190. Gu X, Olson CE, Spitzer NC. Spontaneous neuronal calcium spikes and waves during early differentiation. J Neurosci. 14:6325-6335. [PubMed: 7965039]

191. Guerrero A, Darszon A. Egg jelly triggers a calcium influx which inactivates and is inhibited by calmodulin antagonists in the sea urchin sperm. Biochim Biophys Acta. 1989; 980:109-116. [PubMed: 2923893]

192. Guerrier P, Leclerc-David C, Moreau M. Evidence for the involvement of internal calcium stores during serotonin-induced meiosis reinitation in oocytes of the bivalve mollusc Ruditapes philippinarum. Dev Biol. 1993; 159:474-484. [PubMed: 8405672]

193. Guerrier P, Moreau M, Meijer L, Mazzei G, Vilain JP, Dube F. The role of calcium in meiosis reinitiation. Prog Clin Biol Res. 1982

194. Gurdon JB. The generation of diversity and pattern in animal development. Cell. 1992; 68:185199. [PubMed: 1733498]

195. Gurdon JB, Hopwood ND, Taylor MV. Mechanisms of gene activation in early vertebrate development. C R Acad Sci III. 1992; 314:51-53. [PubMed: 1422906]

196. Hafner M, Petzelt C, Nobiling R, Pawley JB, Kramp D, Schatten G. Wave of free calcium at fertilization in the sea urchin egg visualized with fura-2. Cell Motil Cytoskeleton. 1988; 9:271277. [PubMed: 3365773]

197. Halet G, Tunwell R, Balla T, Swann K, Carroll J. The dynamics of plasma membrane PtdIns(4,5) $\mathrm{P}_{2}$ at fertilization of mouse eggs. J Cell Sci. 2002; 115:2139-2149. [PubMed: 11973355]

198. Hamaguchi Y, Hiramoto Y. Activation of sea urchin eggs by microinjection of calcium buffers. Exp Cell Res. 1981; 134:171-179. [PubMed: 6788576]

199. Hamaguchi Y, Mabuchi I. Accumulation of fluorescently labeled actin in the cortical layer in sea urchin eggs after fertilization. Cell Motil Cytoskeleton. 1988; 9:153-163. [PubMed: 3359492]

200. Han JK, Fukami K, Nuccitelli R. Reducing inositol lipid hydrolysis, Ins(1,4,5)P receptor availability, or $\mathrm{Ca}^{2+} 3$ gradients lengthens the duration of the cell-cycle In Xenopus Laevis blastomeres. J Cell Biol. 1992; 116:147-156. [PubMed: 1309810] 
201. Harland R, Gerhart J. Formation and function of Spemann's organizer. Annu Rev Cell Dev Biol. 1997; 13:611-667. [PubMed: 9442883]

202. Harrison PK, Falugi C, Angelini C, Whitaker M. Muscarinic signalling affects intracellular calcium concentration during the first cell cycle of sea urchin embryos. Cell Calcium. 2002; 31:289-297. [PubMed: 12098218]

203. Hedgepeth CM, Conrad LJ, Zhang J, Huang HC, Lee VM, Klein PS. Activation of the Wnt signaling pathway: a molecular mechanism for lithium action. Dev Biol. 1997; 185:82-91. [PubMed: 9169052]

204. Heilbrunn, LV. The colloid chemistry of protoplasm. Verlag von Gebrueder Borntraeger; Berlin: 1928.

205. Heinecke JW, Meier KE, Lorenzen JA, Shapiro BM. A specific requirement for protein kinase C in activation of the respiratory burst oxidase of fertilization. J Biol Chem. 1990; 265:7717-7720. [PubMed: 2335502]

206. Heinecke JW, Shapiro BM. Protein kinase C activates the respiratory burst of fertilization, but not cortical granule exocytosis, in ionophore-stimulated sea urchin eggs. Dev Biol. 1990; 142:216223. [PubMed: 2227097]

207. Heisenberg CP, Tada M, Rauch G-J, Saúde L, Concha ML, Geisler R, Stemple D, Smith JC, Wilson SW. Silberblick/Wnt11 mediates convergent extension movements during zebrafish gastrulation. Nature. 2000; 405:76-81. [PubMed: 10811221]

208. Henson JH, Begg DA, Beaulieu SM, Fishkind DJ, Bonder EM, Terasaki M, Lebeche D, Kaminer B. A calsequestrin-like protein in the endoplasmic reticulum of the sea urchin: localization and dynamics in the egg and first cell cycle embryo. J Cell Biol. 1989; 109:149-161. [PubMed: 2663877]

209. Hepler PK. Calcium and mitosis. Int Rev Cytol. 1992

210. Hesketh TR, Moore JP, Morris JD, Taylor MV, Rogers J, Smith GA, Metcalfe JC. A common sequence of calcium and $\mathrm{pH}$ signals in the mitogenic stimulation of eukaryotic cells. Nature. 1985; 313:481-484. [PubMed: 3918272]

211. Hiiragi T, Solter D. First cleavage plane in the mouse is not predetermined but defined by the topology of the two apposing pronuclei. Nature. 2004; 430:360-364. [PubMed: 15254539]

212. Hiraoka D, Hori-Oshima S, Fukuhara T, Tachibana K, Okumura E, Kishimoto T. PDK1 is required for the hormonal signaling pathway leading to meiotic resumption in starfish oocytes. Dev Biol. 2004; 276:330-336. [PubMed: 15581868]

213. Hirose K, Kadowaki S, Tanabe M, Takeshima H, Lino M. Spatiotemporal dynamics of inositol 1,4,5-triphosphate that underlies complex $\mathrm{Ca}^{2+}$ metabolism patterns. Science. 1999; 284:15271530. [PubMed: 10348740]

214. Hohenegger M, Suko J, Gscheidlinger R, Drobny H, Zidar A. Nicotinic acid-adenine dinucleotide phosphate activates the skeletal muscle ryanodine receptor. Biochem J. 2002; 367:423-431. [PubMed: 12102654]

215. Holtfreter J. A study of the mechanics of gastrulation, part II. J Exp Zool. 1944; 95:171-212.

216. Homa ST. Calcium and meiotic maturation of the mammalian oocyte. Mol Reprod Dev. 1995; 40:122-134. [PubMed: 7702866]

217. Homa ST, Carroll J, Swann K. The role of calcium in mammalian oocyte maturation and egg activation. Hum Reprod. 1993; 8:1274-1281. [PubMed: 8408526]

218. Homa ST, Swann K. A cytosolic sperm factor triggers calcium oscillations and membrane hyperpolarizations in human oocytes. Hum Reprod. 1994; 9:2356-2361. [PubMed: 7714158]

219. Howell KP, Skipwith A, Galione A, Eckberg WR. Phospholipase C-dependent $\mathrm{Ca}^{2+}$ release by worm and mammal sperm factors. Biochem Biophys Res Commun. 2003; 307:47-51. [PubMed: 12849979]

220. Hunt T. Cyclins and their partners: from a simple idea to complicated reality. Semin Cell Biol. 1991; 2:213-222. [PubMed: 1842340]

221. Hyslop LA, Carroll M, Nixon VL, McDougall A, Jones KT. Simultaneous measurement of intracellular nitric oxide and free calcium levels in chordate eggs demonstrates that nitric oxide has no role at fertilization. Dev Biol. 2001; 234:216-230. [PubMed: 11356031] 
222. Igusa Y, Miyazaki S, Yamashita N. Periodic hyperpolarizing responses in hamster and mouse eggs fertilized with mouse sperm. J Physiol. 1983

223. Iino S, Cui Y, Galione A, Terrar DA. Actions of cADP-ribose and its antagonists on contraction in guinea pig. Circulation Res. 1997; 81:879-884. [PubMed: 9351463]

224. Insall RH, Weiner OD. $\mathrm{PIP}_{3}$, PIP , and cell movement--similar messages, different meanings? Dev Cell. 2001; 1:743-747. [PubMed: 11740936]

225. Itoh K, Sokol SY. Axis determination by inhibition of Wnt signalling in Xenopus. Genes Dev. 1999; 13:2328-2336. [PubMed: 10485853]

226. Iwao Y, Fujimura T. Activation of Xenopus eggs by RGD-containing peptides accompanied by intracellular $\mathrm{Ca}^{2+}$ release. Dev Biol. 1996; 177:558-567. [PubMed: 8806831]

227. Iwasaki H, Chiba K, Uchiyama T, Yoshikawa F, Suzuki F, Ikeda M, Furuichi T, Mikoshiba K. Molecular characterization of the starfish inositol 1,4,5-trisphosphate receptor and its role during oocyte maturation and fertilization. J Biol Chem. 2002; 277:2763-2772. [PubMed: 11687583]

228. Jaffe L. On the conservation of fast calcium wave speeds. Cell Calcium. 2002; 32:217-229. [PubMed: 12379182]

229. Jaffe LA. Fast block to polyspermy in sea urchin eggs is electrically mediated. Nature. 1976; 261:68-71. [PubMed: 944858]

230. Jaffe LA, Cross NL. Electrical regulation of sperm-egg fusion. Annu Rev Physiol. 1986

231. Jaffe LA, Giusti AF, Carroll DJ, Foltz KR. $\mathrm{Ca}^{2+}$ signalling during fertilization of echinoderm eggs. Semin Cell Dev Biol. 2001; 12:45-51. [PubMed: 11162746]

232. Jaffe LA, Sharp AP, Wolf DP. Absence of an electrical polyspermy block in the mouse. Dev Biol. 1983; 96:317-323. [PubMed: 6832474]

233. Jaffe LF. Calcium explosions as triggers of development. Ann N Y Acad Sci. 1980

234. Jaffe LF. Calcium waves and development. Ciba Found Symp. 1995; 188:4-12. [PubMed: 7587622]

235. Jaffe LF. On the validity of the sperm conduit model of fertilization. Bioessays. 1997; 19:835836. [PubMed: 9297975]

236. Jaffe LF. Organization of early development by calcium patterns. Bioessays. 1999; 21:657-667. [PubMed: 10440862]

237. Jaffe LF. The path of calcium in cytosolic calcium oscillations: a unifying hypothesis. Proc Natl Acad Sci U S A. 1991; 88:9883-9887. [PubMed: 1946414]

238. Jaffe LF, Crèton R. On the conservation of calcium wave speeds. Cell Calcium. 1998; 24:1-8. [PubMed: 9793683]

239. Jeffery WR, Swalla BJ. The myoplasm of ascidian eggs: a localized cytoskeletal domain with multiple roles in embryonic development. Semin Cell Biol. 1990; 1:373-381. [PubMed: 2102391]

240. Jellerette T, He CL, Wu H, Parys JB, Fissore RA. Down-regulation of the inositol 1,4,5trisphosphate receptor in mouse eggs following fertilization or parthenogenetic activation. Dev Biol. 2000; 223:238-250. [PubMed: 10882513]

241. Johnson JD, Epel D. Intracellular $\mathrm{pH}$ and activation of sea urchin eggs after fertilisation. Nature. 1976; 262:661-664. [PubMed: 8729]

242. Jones KT. $\mathrm{Ca}^{2+}$ oscillations in the activation of the egg and development of the embryo in mammals. Int J Dev Biol. 1998; 42:1-10. [PubMed: 9496781]

243. Jones KT, Carroll J, Whittingham DG. Ionomycin, thapsigargin, ryanodine, and sperm induced $\mathrm{Ca}^{2+}$ release increase during meiotic maturation of mouse oocytes. J Biol Chem. 1995; 270:6671-6677. [PubMed: 7896808]

244. Jones KT, Matsuda M, Parrington J, Katan M, Swann K. Different $\mathrm{Ca}^{2+}$-releasing abilities of sperm extracts compared with tissue extracts and phospholipase $\mathrm{C}$ isoforms in sea urchin egg homogenate and mouse eggs. Biochem J. 2000; 346:743-749. [PubMed: 10698702]

245. Jones KT, Nixon VL. Sperm-induced $\mathrm{Ca}^{2+}$ oscillations in mouse oocytes and eggs can be mimicked by photolysis of caged inositol 1,4,5-trisphosphate: evidence to support a continuous low level production of inositol 1, 4,5-trisphosphate during mammalian fertilization. Dev Biol. 2000; 225:1-12. [PubMed: 10964460] 
246. Jones KT, Soeller C, Cannell MB. The passage of $\mathrm{Ca}^{2+}$ and fluorescent markers between the sperm and egg after fusion in the mouse. Development. 1998; 125:4627-4635. [PubMed: 9806912]

247. Jones KT, Whittingham DG. A comparison of sperm- and IP -induced $\mathrm{Ca}^{2+} 3$ release in activated and aging mouse oocytes. Dev Biol. 1996; 178:229-237. [PubMed: 8812125]

248. Kaji K, Oda S, Shikano T, Ohnuki T, Uematsu Y, Sakagami J, Tada N, Miyazaki S, Kudo A. The gamete fusion process is defective in eggs of Cd9-deficient mice. Nat Genet. 2000; 24:279-282. [PubMed: 10700183]

249. Kaltschmidt JA, Brand AH. Asymmetric cell division: microtubule dynamics and spindle asymmetry. J Cell Sci. 2002; 115:2257-2264. [PubMed: 12006610]

250. Kamei N, Swanson WJ, Glabe CG. A rapidly diverging EGF protein regulates species-specific signal transduction in early sea urchin development. Dev Biol. 2000; 225:267-276. [PubMed: 10985849]

251. Kamel LC, Bailey J, Schoenbaum L, Kinsey W. Phosphatidylinositol metabolism during fertilization in the sea urchin egg. Lipids. 1985; 20:350-356. [PubMed: 4040596]

252. Kawahara H, Yokosawa H. Intracellular calcium mobilization regulates the activity of $26 \mathrm{~S}$ proteasome during the metaphase-anaphase transition in the ascidian meiotic cell cycle. Dev Biol. 1994; 166:623-633. [PubMed: 7813781]

253. Kawakami Y, Raya A, Raya MR, Rodriguez-Esteban C, Belmonte JCI. Retinoic acid signalling links left-right asymmetric patterning and bilaterally symmetric somitogenesis in the zebrafish embryo. Nature. 2005; 435:165-171. [PubMed: 15889082]

254. Keating TJ, Cork RJ, Robinson KR. Intracellular free calcium oscillations in normal and cleavage-blocked embryos and artificially activated eggs of Xenopus laevis. J Cell Sci. 1994; 107:2229-2237. [PubMed: 7983182]

255. Keizer J, Li YX, Stojilkovic S, Rinzel J. InsP -induced $\mathrm{Ca}^{2+} 3$ excitability of the endoplasmic reticulum. Mol Biol Cell. 1995; 6:945-951. [PubMed: 7579710]

256. Keizer J, Smith GD. Spark-to-wave transition: saltatory transmission of calcium waves in cardiac myocytes. Biophys Chem. 1998; 72:87-100. [PubMed: 9652087]

257. Keller R. Heading away from the rump. Nature. 2004; 430:305-306. [PubMed: 15254524]

258. Keller R, Davidson L, Edlund A, Elul T, Ezin M, Shook D, Skoglund P. Mechanisms of convergence and extension by cell intercalation. Philos Trans R Soc Lond B Biol Sci. 2000; 355:897-922. [PubMed: 11128984]

259. Kiehart DP, Mabuchi I, Inoue S. Evidence that myosin does not contribute to force production in chromosome movement. J Cell Biol. 1982; 94:165-178. [PubMed: 6181080]

260. Kilian B, Mansukoski H, Barbosa FC, Ulrich F, Tada M, Heisenberg CP. The role of Ppt/Wnt5 in regulation cell shape and movement during zebrafish gastrulation. Mech Dev. 2003; 120:467476. [PubMed: 12676324]

261. Kimmel CB, Ballard WW, Kimmel SR, Ullmann B, Schilling TF. Stages of embryonic development of the zebrafish. Dev Dyn. 1995; 203:253-310. [PubMed: 8589427]

262. Kinsey WH. Biphasic activation of Fyn kinase upon fertilization of the sea urchin egg. Dev Biol. 1996; 174:281-287. [PubMed: 8631500]

263. Kinsey WH. Regulation of tyrosine-specific kinase activity at fertilization. Dev Biol. 1984; 105:137-143. [PubMed: 6432606]

264. Kinsey WH, Shen SS. Role of the Fyn kinase in calcium release during fertilization of the sea urchin egg. Dev Biol. 2000; 225:253-264. [PubMed: 10964479]

265. William, H Kinsey; Wu, W.; Macgregor, E. Activation of Src-family PTK activity at fertilization: role of the SH2 domain. Dev Biol. 2003; 264:255-262. [PubMed: 14623246]

266. Kishimoto T. Activation of MPF at meiosis reinitiation in starfish oocytes. Dev Biol. 1999; 214:1-8. [PubMed: 10491252]

267. Kishimoto T, Usui N, Kanatani H. Breakdown of starfish ovarian follicle induced by maturationpromoting factor. Dev Biol. 1984; 101:28-34. [PubMed: 6363159]

268. Klein PS, Melton DA. A molecular mechanism for the effect of lithium on development. Proc Natl Acad Sci U S A. 1996; 93:8455-8459. [PubMed: 8710892] 
269. Kline D. Attributes and dynamics of the endoplasmic reticulum in mammalian eggs. Curr Top Dev Biol. 2000

270. Kline D. Calcium-dependent events at fertilization of the frog egg: injection of a calcium buffer blocks ion channel opening, exocytosis, and formation of pronuclei. Dev Biol. 1988; 126:346361. [PubMed: 2450795]

271. Kline D, Kline JT. Repetitive calcium transients and the role of calcium in exocytosis and cell cycle activation in the mouse egg. Dev Biol. 1992; 149:80-89. [PubMed: 1728596]

272. Kline D, Kline JT. Thapsigargin activates a calcium influx pathway in the unfertilized mouse egg and suppresses repetitive calcium transients in the fertilized egg. J Biol Chem. 1992; 267:1762417630. [PubMed: 1387638]

273. Kline D, Kopf GS, Muncy LF, Jaffe LA. Evidence for the involvement of a pertussis toxininsensitive G-protein in egg activation of the frog, Xenopus laevis. Dev Biol. 1991; 143:218229. [PubMed: 1899403]

274. Kline D, Mehlmann L, Fox C, Terasaki M. The cortical endoplasmic reticulum (ER) of the mouse egg: localization of ER clusters in relation to the generation of repetitive calcium waves. Dev Biol. 1999; 215:431-442. [PubMed: 10545249]

275. Kline D, Stewart-Savage J. The timing of cortical granule fusion, content dispersal, and endocytosis during fertilization of the hamster egg: an electrophysiological and histochemical study. Dev Biol. 1994; 162:277-287. [PubMed: 8125193]

276. Kline JT, Kline D. Regulation of intracellular calcium in the mouse egg: evidence for inositol trisphosphate-induced calcium release, but not calcium-induced calcium release. Biol Reprod. 1994; 50:193-203. [PubMed: 8312443]

277. Knott JG, Kurokawa M, Fissore RA. Release of the $\mathrm{Ca}^{2+}$ oscillation-inducing sperm factor during mouse fertilization. Dev Biol. 2003; 260:536-547. [PubMed: 12921751]

278. Kono T, Carroll J, Swann K, Whittingham DG. Nuclei from fertilized mouse embryos have calcium-releasing activity. Development. 1995; 121:1123-1128. [PubMed: 7743925]

279. Kono T, Jones KT, Bos-Mikich A, Whittingham DG, Carroll J. A cell cycle-associated change in $\mathrm{Ca}^{2+}$ releasing activity leads to the generation of $\mathrm{Ca}^{2+}$ transients in mouse embryos during the first mitotic division. J Cell Biol. 1996; 132:915-923. [PubMed: 8603922]

280. Kouchi Z, Fukami K, Shikano T, Oda S, Nakamura Y, Takenawa T, Miyazaki S. Recombinant phospholipase $\mathrm{C} \zeta$ has high $\mathrm{Ca}^{2+}$ sensitivity and induces $\mathrm{Ca}^{2+}$ oscillations in mouse eggs. J Biol Chem. 2004; 279:10408-10412. [PubMed: 14701816]

281. Kramer KL, Barnette JE, Yost HJ. PKC $\gamma$ regulates syndecan-2 inside-out signalling during Xenopus left-right development. Cell. 2002; 111:981-990. [PubMed: 12507425]

282. Luke, T Krebs; Iwai, N.; Nonaka, S.; Ian, C Welsh; Lan, Y.; Jiang, R.; Saijoh, Y.; Timothy, P O'Brien; Hamada, H.; Gridley, T. Notch signaling regulates left-right asymmetry determination by inducing Nodal expression. Genes Dev. 2003; 17:1207-1212. [PubMed: 12730124]

283. Kubota HY, Yoshimoto Y, Hiramoto Y. Oscillations of intracellular free calcium in cleaving and cleavage-arrested embryos of Xenopus laevis. Dev Biol. 1993; 160:512-518. [PubMed: 8253280]

284. Kühl M. Non-canonical Wnt signalling in Xenopus: regulation of axis formation and gastrulation. Semin Cell Dev Biol. 2002; 13:243-249. [PubMed: 12137733]

285. Kühl M, Geis K, Sheldahl LC, Pukrop T, Moon RT, Wedlich D. Antagonistic regulation of convergent extension movements in Xenopus by Wnt/beta-catenin and $\mathrm{Wnt} / \mathrm{Ca}^{2+}$ signaling. Mech Dev. 2001; 106:61-76. [PubMed: 11472835]

286. Kühl M, Sheldahl LC, Malbon CC, Moon RT. $\mathrm{Ca}^{2+} /$ calmodulin-dependent protein kinase II is stimulated by Wnt and frizzled homologs and promotes ventral cell fates in Xenopus. J Biol Chem. 2000; 275:12701-12711. [PubMed: 10777564]

287. Kumakiri J, Oda S, Kinoshita K, Miyazaki S. Involvement of Rho family G protein in the cell signaling for sperm incorporation during fertilization of mouse eggs: inhibition by Clostridium difficile toxin B. Dev Biol. 2003; 260:522-535. [PubMed: 12921750]

288. Kume S, Inoue T, Mikoshiba K. Gas family G-proteins activate IP $-\mathrm{Ca}^{2+} 3$ signalling via G $\beta \gamma$ and transduce ventralizing signals. Dev Biol. 2000; 226:88-103. [PubMed: 10993676] 
289. Kume S, Muto A, Inoue T, Suga K, Okano H, Mikoshiba K. Role of inositol 1,4,5-trisphosphate receptor in ventral signaling in Xenopus embryos. Science. 1997; 278:1940-1943. [PubMed: 9395395]

290. Kume S, Muto A, Okano H, Mikoshiba K. Developmental expression of the inositol 1,4,5trisphosphate receptor and localization of inositol 1,4,5-trisphosphate during early embryogenesis in Xenopus laevis. Mech Dev. 1997; 66:157-168. [PubMed: 9376319]

291. Kume S, Saneyoshi T, Mikoshiba K. Desensitization of IP -induced $\mathrm{Ca}^{2+} 3$ release by overexpression of a consitutively active Gqa protein converts ventral to dorsal fate in Xenopus early embryos. Dev Growth Differ. 2000; 42:327-355. [PubMed: 10969732]

292. Kuo RC, Baxter GT, Thompson SH, Stricker SA, Patton C, Bonaventura J, Epel D. NO is necessary and sufficient for egg activation at fertilization. Nature. 2000; 406:633-636. [PubMed: 10949304]

293. Kuroda R, Kontani K, Kanda Y, Katada T, Nakano T, Satoh Y, Suzuki N, Kuroda H. Increase of cGMP, cADP-ribose and inositol 1,4,5-trisphosphate preceding $\mathrm{Ca}^{2+}$ transients in fertilization of sea urchin eggs. Development. 2001; 128:4405-4414. [PubMed: 11714667]

294. Kurokawa D, Akasaka K, Mitsunaga-Nakatsubo K, Shimada H. Cloning of cyclin E cDNA of the sea urchin, Hemicentrotus pulcherrimus. Zoolog Sci. 1997; 14:791-794. [PubMed: 9450391]

295. Kyozuka K, Deguchi R, Mohri T, Miyazaki S. Injection of sperm extract mimics spatiotemporal dynamics of $\mathrm{Ca}^{2+}$ responses and progression of meiosis at fertilization of ascidian oocytes. Development. 1998; 125:4099-4105. [PubMed: 9735370]

296. Lambert C, Goudeau H, Franchet C, Lambert G, Goudeau M. Ascidian eggs block polyspermy by two independent mechanisms: one at the egg plasma membrane, the other involving the follicle cells. Mol Reprod Dev. 1997; 48:137-143. [PubMed: 9266770]

297. Larman MG, Saunders CM, Carroll J, Lai FA, Swann K. Cell cycle-dependent $\mathrm{Ca}^{2+}$ oscillations in mouse embryos are regulated by nuclear targeting of PLCS. J Cell Sci. 2004; 117:2513-2521. [PubMed: 15159452]

298. Lawrence Y, Ozil JP, Swann K. The effects of a $\mathrm{Ca}^{2+}$ chelator and heavy-metal-ion chelators upon $\mathrm{Ca}^{2+}$ oscillations and activation at fertilization in mouse eggs suggest a role for repetitive $\mathrm{Ca}^{2+}$ increases. Biochem J. 1998; 335:335-342. [PubMed: 9761732]

299. Lawrence Y, Whitaker M, Swann K. Sperm-egg fusion is the prelude to the initial $\mathrm{Ca}^{2+}$ increase at fertilization in the mouse. Development. 1997; 124:233-241. [PubMed: 9006083]

300. Lawrence YM, Cuthbertson KS. Thapsigargin induces cytoplasmic free $\mathrm{Ca}^{2+}$ oscillations in mouse oocytes. Cell Calcium. 1995; 17:154-164. [PubMed: 7736564]

301. Leckie MP, Empson RM, Bechetti A, Thomas J, Galione A, Whitaker M. The NO pathway acts late during the fertilization response in sea urchin eggs. J Biol Chem. 2003; 278:12247-12254. [PubMed: 12540836]

302. Leclerc C, Daguzan C, Nicolas MT, Charbret C, Duprat AM, Moreau M. L-type calcium channel activation controls the in vivo transduction of neuralizing signals in the amphibian embryos. Mech Dev. 1997; 64:105-110. [PubMed: 9232601]

303. Leclerc C, Duprat AM, Moreau M. In vivo labelling of L-type $\mathrm{Ca}^{2+}$ channels by fluorescent dihydropyridine: correlation between ontogenesis of the channels and the acquisition of neural competence in ectoderm cells from Pleurodeles walt embryos. Cell Calcium. 1995; 17:216-224. [PubMed: 7542570]

304. Leclerc C, Duprat AM, Moreau M. Noggin upregulates fos expression by a calcium mediated pathway in amphibian embryos. Dev Growth Differ. 1999; 41:227-238. [PubMed: 10223719]

305. Leclerc C, Webb SE, Daguzan C, Moreau M, Miller AL. Imaging patterns of calcium transients during neural induction in Xenopus laevis embryos. J Cell Sci. 2000; 113:3519-3529. [PubMed: 10984442]

306. Lee HC. Mechanisms of calcium signaling by cyclic ADP-ribose and NAADP. Physiol Rev. 1997; 77:1133-1164. [PubMed: 9354813]

307. Lee HC. Physiological functions of cyclic ADP-ribose and NAADP as calcium messengers. Annu Rev Pharmacol Toxicol. 2001

308. Lee HC, Aarhus R. A derivative of NADP mobilizes calcium stores insensitive to inositol trisphosphate and cyclic ADP-ribose. J Biol Chem. 1995; 270:2152-2157. [PubMed: 7836444] 
309. Lee HC, Aarhus R. Functional visualization of the separate but interacting calcium stores sensitive to NAADP and cyclic ADP-ribose. J Cell Sci. 2000; 113:4413-4420. [PubMed: 11082034]

310. Lee HC, Aarhus R, Graeff R, Gurnack ME, Walseth TF. Cyclic ADP ribose activation of the ryanodine receptor is mediated by calmodulin. Nature. 1994; 370:307-309. [PubMed: 8035880]

311. Lee HC, Aarhus R, Graeff RM. Sensitization of calcium-induced calcium release by cyclic ADPribose and calmodulin. J Biol Chem. 1995; 270:9060-9066. [PubMed: 7721819]

312. Lee HC, Aarhus R, Walseth TF. Calcium mobilization by dual receptors during fertilization of sea urchin eggs. Science. 1993; 261:352-355. [PubMed: 8392749]

313. Lee HC, Galione A, Walseth TF. Cyclic ADP-ribose: metabolism and calcium mobilizing function. Vitam Horm. 1994

314. Lee KW, Ho SM, Wong CH, Webb SE, Miller AL. Characterization of mid-spindle microtubules during furrow positioning in early cleavage period zebrafish embryos. Zygote. 2004; 12:221230. [PubMed: 15521712]

315. Lee KW, Webb SE, Miller AL. $\mathrm{Ca}^{2+}$ released via $\mathrm{InsP}_{3}$ receptors is required for furrow deepening during cytokinesis in zebrafish embryos. Int J Dev Biol. 2003; 47:411-421. [PubMed: 14584779]

316. Lee SJ, Christenson L, Martin T, Shen SS. The cyclic GMP-mediated calcium release pathway in sea urchin eggs is not required for the rise in calcium during fertilization. Dev Biol. 1996; 180:324-335. [PubMed: 8948594]

317. Lee SJ, Shen SS. The calcium transient in sea urchin eggs during fertilization requires the production of inositol 1,4,5-trisphosphate. Dev Biol. 1998; 193:195-208. [PubMed: 9473324]

318. Lemmon M, Ferguson KM, O’Brian R, Sigler PB, Schlessinger J. Specific and high-affinity binding of inositol phosphates to an isolated pleckstrin homology domain. Proc Natl Acad Sci. 1995; 92:10472-10476. [PubMed: 7479822]

319. Leung CF, Webb SE, Miller AL. Calcium transients accompany ooplasmic segregation in zebrafish embryos. Dev Growth Differ. 1998; 40:313-326. [PubMed: 9639359]

320. Leung CF, Webb SE, Miller AL. On the mechanism of ooplasmic segregation in single-cell zebrafish embryos. Dev Growth Differ. 2000; 42:29-40. [PubMed: 10831041]

321. Levasseur M, McDougall A. Sperm-induced calcium oscillations at fertilisation in ascidians are controlled by cyclin B1-dependent kinase activity. Development. 2000; 127:631-641. [PubMed: 10631183]

322. Levin M, Mercola M. Gap junction-mediated transfer of left-right patterning signals in the early chick blastoderm is upstream of Shh asymmetry in the node. Development. 1999; 126:47034714. [PubMed: 10518488]

323. Levin M, Mercola M. Gap junctions are involved in the early generation of left right asymmetry. Dev Biol. 1998; 203:90-105. [PubMed: 9806775]

324. Levin M, Thorlin T, Robinson K, Nogi T, Mercola M. Asymmetries in $\mathrm{H}^{+} / \mathrm{K}^{+}$ATPase and cell membrane potential comprise a very early step in left-right patterning. Cell. 2002; 111:77-89. [PubMed: 12372302]

325. Li ST, Huang XY, Sun FZ. Flowering plant sperm contains a cytosolic soluble protein factor which can trigger calcium oscillations in mouse eggs. Biochem Biophys Res Commun. 2001; 287:56-59. [PubMed: 11549252]

326. Li W, Llopis J, Whitney M, Zlokarnik G, Tsien RY. Cell-permeant caged InsP ester shows that $\mathrm{Ca}^{2+} 3$ spike frequency can optimize gene expression. Nature. 1998; 392:936-941. [PubMed: 9582076]

327. Lillie, FR. Problems of fertilization. University of Chicago Press; Chicago, USA: 1919.

328. Lim D, Ercolano E, Kyozuka K, Nusco Gilda A, Moccia F, Lange K, Santella L. The M-phasepromoting factor modulates the sensitivity of the $\mathrm{Ca}^{2+}$ stores to inositol 1,4,5-trisphosphate via the actin cytoskeleton. J Biol Chem. 2003; 278:42505-42514. [PubMed: 12867432]

329. Lim D, Kyozuka K, Gragnaniello G, Carafoli E, Santella L. NAADP ${ }^{+}$initiates the $\mathrm{Ca}^{2+}$ response during fertilization of starfish oocytes. FASEB J. 2001; 15:2257-2267. [PubMed: 11641253]

330. Lim D, Lange K, Santella L. Activation of oocytes by latrunculin A. FASEB J. 2002; 16:10501056. [PubMed: 12087066] 
331. Lipp P, Thomas D, Berridge MJ, Bootman MD. Nuclear calcium signalling by individual cytoplasmic calcium puffs. EMBO J. 1997; 16:7166-7173. [PubMed: 9384593]

332. Livingston BT, Wilt FH. Lithium evokes expression of vegetal-specific molecules in the animal blastomeres of sea urchin embryos. Proc Natl Acad Sci U S A. 1989; 86:3669-3673. [PubMed: 2726745]

333. Livingston BT, Wilt FH. Phorbol esters alter cell fate during development of sea urchin embryos. J Cell Biol. 1992; 119:1641-1648. [PubMed: 1469053]

334. Longo FJ, Lynn JW, McCulloh DH, Chambers EL. Correlative ultrastructural and electrophysiological studies of sperm egg interactions of the sea urchin, Lytechinus variegatus. Dev Biol. 1986; 118:155-166. [PubMed: 3770296]

335. Lorca T, Cruzalegui FH, Fesquet D, Cavadore JC, Mery J, Means A, Doree M. Calmodulindependent protein kinase II mediates inactivation of MPF and CSF upon fertilization of Xenopus eggs. Nature. 1993; 366:270-273. [PubMed: 8232587]

336. Lorca T, Galas S, Fesquet D, Devault A, Cavadore JC, Doree M. Degradation of the protooncogene product $\mathrm{p} 39 \mathrm{mos}$ is not necessary for cyclin proteolysis and exit from meiotic metaphase: requirement for a $\mathrm{Ca}^{2+}$-calmodulin dependent event. EMBO J. 1991; 10:2087-2093. [PubMed: 1829675]

337. Lu P, Yu V, Li CJ, Chang DC. Calcium signals are involved in initiating the cleavage furrow formation in zebrafish embryo. Mol Biol Cell. 1997; 8:167-167.

338. Lu W, Fan X, Basora N, Babakhanlou H, Law T, Rifai N, Harris PC, Perez-Atayde AR, Rennke HG, Zhou J. Late onset of renal and hepatic cysts in Pkd1-targeted heterozygotes. Nat Genet. 1999; 21:160-161. [PubMed: 9988265]

339. Luke NH, Killian CE, Livingston BT. Spfkh1 encodes a transcription factor implicated in gut formation during sea urchin development. Dev Growth Differ. 1997; 39:285-294. [PubMed: 9227895]

340. Luo Y, Peter M Vassilev, Li X, Kawanabe Y, Zhou J. Native polycystin 2 functions as a plasma membrane $\mathrm{Ca}^{2+}$-permeable cation channel in renal epithelia. Mol Cell Biol. 2003; 23:26002607. [PubMed: 12640140]

341. Lynn JW, Chambers EL. Voltage clamp studies of fertilization in sea urchin eggs. I. Effect of clamped membrane-potential on sperm entry, activation, and development. Dev Biol. 1984; 102:98-109. [PubMed: 6698308]

342. Lynn JW, McCulloh DH, Chambers EL. Voltage clamp studies of fertilization in sea urchin eggs. II. Current patterns in relation to sperm entry, nonentry, and activation. Dev Biol. 1988; 128:305-323. [PubMed: 3396763]

343. Machaca K, Haun S. Induction of maturation-promoting factor during Xenopus oocyte maturation uncouples $\mathrm{Ca}(2+)$ store depletion from store-operated $\mathrm{Ca}^{2+}$ entry. J Cell Biol. 2002; 156:75-85. [PubMed: 11781335]

344. Maller JL. Oocyte maturation in amphibians. Dev Biol. 1985; 1:289-311.

345. Maller JL. Recurring themes in oocyte maturation. Biol Cell. 1998; 90:453-460. [PubMed: 9923070]

346. Marangos P, FitzHarris G, Carroll J. $\mathrm{Ca}^{2+}$ oscillations at fertilization in mammals are regulated by the formation of pronuclei. Development. 2003; 130:1461-1472. [PubMed: 12588860]

347. Marchant J, Callamaras N, Parker I. Initiation of IP -mediated $\mathrm{Ca}^{2+} 3$ waves in Xenopus oocytes. EMBO J. 1999; 18:5285-5299. [PubMed: 10508162]

348. Markoulaki S, Matson S, Allison L Abbott, Ducibella T. Oscillatory CaMKII activity in mouse egg activation. Dev Biol. 2003; 258:464-474. [PubMed: 12798302]

349. Maslanski JA, Leshko L, Busa WB. Lithium-sensitive production of inositol phosphates during amphibian embryonic mesoderm induction. Science. 1992; 256:243-245. [PubMed: 1314424]

350. Mazia D, Schatten G, Steinhardt R. Turning on of activities in unfertilized sea urchin eggs: correlation with changes of the surface. Proc Natl Acad Sci U S A. 1975; 72:4469-4473. [PubMed: 1060127]

351. McAvey BA, Wortzman GB, Williams CJ, Evans JP. Involvement of calcium signaling and the actin cytoskeleton in the membrane block to polyspermy in mouse eggs. Biol Reprod. 2002; 67:1342-1352. [PubMed: 12297554] 
352. McCulloh DH, Chambers EL. Fusion of membranes during fertilization - Increases of the seaurchin egg's membrane capacitance and membrane conductance at the site of contact with the sperm. J Gen Physiol. 1992; 99:137-175. [PubMed: 1613481]

353. McCulloh DH, Ivonnet PI, Landowne D, Chambers EL. Calcium influx mediates the voltagedependence of sperm entry into sea urchin eggs. Dev Biol. 2000; 223:449-462. [PubMed: 10882529]

354. McCulloh DH, Lynn JW, Chambers EL. Membrane depolarization facilitates sperm entry, large fertilization cone formation, and prolonged current responses in sea-urchin oocytes. Dev Biol. 1987; 124:177-190. [PubMed: 3666304]

355. McDougall A, Gillot I, Whitaker M. Thimerosal reveals calcium-induced calcium release in unfertilised sea urchin eggs. Zygote. 1993; 1:35-42. [PubMed: 8081800]

356. McDougall A, Levasseur M. Sperm-triggered calcium oscillations during meiosis in ascidian oocytes first pause, restart, then stop: correlations with cell cycle kinase activity. Development. 1998; 125:4451-4459. [PubMed: 9778504]

357. McDougall A, Levasseur M, O’Sullivan AJ, Jones KT. Cell cycle-dependent repetitive Ca ${ }^{2+}$ waves induced by a cytosolic sperm extract in mature ascidian eggs mimic those observed at fertilization. J Cell Sci. 2000; 113:3453-3462. [PubMed: 10984436]

358. McDougall A, Sardet C. Function and characteristics of repetitive calcium waves associated with meiosis. Curr Biol. 1995; 5:318-328. [PubMed: 7780742]

359. McDougall A, Sardet C, Lambert CC. Different calcium-dependent pathways control fertilisationtriggered glycoside release and the cortical contraction in ascidian eggs. Zygote. 1995; 3:251258. [PubMed: 8903794]

360. McDougall A, Shearer J, Whitaker M. The initiation and propagation of the fertilization wave in sea urchin eggs. Biol Cell. 2000; 92:205-214. [PubMed: 11043409]

361. McGrath J, Somlo S, Makova S, Tian X, Brueckner M. Two populations of nodal monocilia initiate left-right asymmetry in the mouse. Cell. 2003; 114:61-73. [PubMed: 12859898]

362. Mehlmann L, Jaffe LA. SH2 domain-mediated activation of a SRC family kinase is not required to initiate $\mathrm{Ca}^{2+}$ release at fertilization in mouse eggs. Reproduction. 2005; 129:557-564. [PubMed: 15855619]

363. Mehlmann LM, Carpenter G, Rhee SG, Jaffe LA. SH2 domain-mediated activation of phospholipase $\mathrm{C} \gamma$ is not required to initiate $\mathrm{Ca}^{2+}$ release at fertilization of mouse eggs. Dev Biol. 1998; 203:221-232. [PubMed: 9806786]

364. Mehlmann LM, Chattopadhyay A, Carpenter G, Jaffe LA. Evidence that phospholipase C from the sperm is not responsible for initiating $\mathrm{Ca}^{2+}$ release at fertilization in mouse eggs. Dev Biol. 2001; 236:492-501. [PubMed: 11476587]

365. Mehlmann LM, Jones TLZ, Jaffe LA. Meiotic arrest in the mouse follicle maintained by a Gs protein in the oocyte. Science. 2002; 297:1343-1345. [PubMed: 12193786]

366. Mehlmann LM, Mikoshiba K, Kline D. Redistribution and increase in cortical inositol 1,4,5trisphosphate receptors after meiotic maturation of the mouse oocyte. Dev Biol. 1996; 180:489498. [PubMed: 8954721]

367. Mehlmann LM, Saeki Y, Tanaka S, Brennan TJ, Evsikov AV, Pendola FL, Knowles BB, Eppig JJ, Jaffe LA. The Gs-linked receptor GPR3 maintains meiotic arrest in mammalian oocytes. Science. 2004; 306:1947-1950. [PubMed: 15591206]

368. Mercola M, Levin M. Left-right asymmetry determination in vertebrates. Annu Rev Cell Dev Biol. 2001; 17:779-805. [PubMed: 11687504]

369. Miller BJ, Georges-Labouesse E, Primakoff P, Myles DG. Normal fertilization occurs with eggs lacking the integrin a6 $\beta 1$ and is CD9-dependent. J Cell Biol. 2000; 149:1289-1296. [PubMed: 10851025]

370. Miller JR, Hocking AM, Brown JD, Moon RT. Mechanism and function of signal transduction by the Wnt/beta-catenin and $\mathrm{Wnt} / \mathrm{Ca}^{2+}$ pathways. Oncogene. 1999; 18:7860-7872. [PubMed: 10630639]

371. Miller JR, Rowning BA, Larabell CA, Yang-Snyder J, Bates RL, Moon RT. Establishment of the dorso-ventral axis in Xenopus coincides with the dorsal enrichment of dishevelled that is dependent on cortical rotation. J Cell Biol. 1999; 146:427-437. [PubMed: 10427095] 
372. Mitsuyama F, Swai T, Carafoli E, Furuichi T, Mikoshiba K. Microinjection of $\mathrm{Ca}^{2+}$ storeenriched microsome fractions to dividing newt eggs induces extra-cleavage furrows via inositol 1,4,5-trisphosphate-induced $\mathrm{Ca}^{2+}$ release. Dev Biol. 1999; 214:160-167. [PubMed: 10491265]

373. Miyazaki S. Calcium signalling during mammalian fertilization. Ciba Found Symp. 1995

374. Miyazaki S. Inositol 1,4,5-trisphosphate-induced calcium release and guanine nucleotide-binding protein-mediated periodic calcium rises in golden hamster eggs. J Cell Biol. 1988; 106:345-353. [PubMed: 3123497]

375. Miyazaki S. Repetitive calcium transients in hamster oocytes. Cell Calcium. 1991; 12:205-216. [PubMed: 2059993]

376. Miyazaki S, Igusa Y. Ca-mediated activation of a K current at fertilization of golden hamster eggs. Proc Natl Acad Sci U S A. 1982; 79:931-935. [PubMed: 6278501]

377. Miyazaki S, Shirakawa H, Nakada K, Honda Y. Essential role of the inositol 1,4,5-trisphosphate receptor/Ca ${ }^{2+}$ release channel in $\mathrm{Ca}^{2+}$ waves and $\mathrm{Ca}^{2+}$ oscillations at fertilization of mammalian eggs. Dev Biol. 1993; 158:62-78. [PubMed: 8392472]

378. Miyazaki S, Yuzaki M, Nakada K, Shirakawa H, Nakanishi S, Nakade S, Mikoshiba K. Block of $\mathrm{Ca}^{2+}$ wave and $\mathrm{Ca}^{2+}$ oscillation by antibody to the inositol 1,4,5-trisphosphate receptor in fertilized hamster eggs. Science. 1992; 257:251-255. [PubMed: 1321497]

379. Mlozdic M. Planar cell polarization: do the same mechanisms regulate Drosophila tissue polarity and vertebrate gastrulation? Trends Genet. 2002; 18:564-571. [PubMed: 12414186]

380. Moccia F, Lim D, Kyozuka K, Santella L. NAADP triggers the fertilization potential in starfish oocytes. Cell Calcium. 2004; 36:515-524. [PubMed: 15488601]

381. Moccia F, Lim D, Gilda A Nusco, Ercolano E, Santella L. NAADP activates a $\mathrm{Ca}^{2+}$ current that is dependent on F-actin cytoskeleton. FASEB J. 2003; 17:1907-1909. [PubMed: 12923070]

382. Moccia F, Nusco GA, Lim D, Ercolano E, Gragnaniello G, Brown ER, Santella L. Ca ${ }^{2+}$ signalling and membrane current activated by cADPr in starfish oocytes. Pflugers Arch. 2003; 446:541-552. [PubMed: 12756567]

383. Mohri T, Hamaguchi Y. Propagation of transient $\mathrm{Ca}^{2+}$ increase in sea urchin eggs upon fertilization and its regulation by microinjecting EGTA solution. Cell Struct Funct. 1991; 16:157-165. [PubMed: 1907218]

384. Mohri T, Ivonnet PI, Chambers EL. Effect on sperm-induced activation current and increase of cytosolic $\mathrm{Ca}^{2+}$ by agents that modify the mobilization of $\mathrm{Ca}^{2+}$ (I) Heparin and pentosan polysulfate. Dev Biol. 1995; 172:139-157. [PubMed: 7589794]

385. Mohri T, Miyazaki S, Shirakawa H, Ikegami S. Sperm-induced local $\left[\mathrm{Ca}^{2+}\right]$ rise separated from the $\mathrm{Ca}^{2+} \mathrm{i}$ wave in sea urchin eggs in the presence of a gamete fusion inhibitor, jaspisin. Development. 1998; 125:293-300. [PubMed: 9486802]

386. Mohri T, Shirakawa H, Oda S, Sato MS, Mikoshiba K, Miyazaki S. Analysis of $\mathrm{Mn}^{2+} / \mathrm{Ca}^{2+}$ influx and release during $\mathrm{Ca}^{2+}$ oscillations in mouse eggs injected with sperm extract. Cell Calcium. 2001; 29:311-325. [PubMed: 11292388]

387. Moon RT. In pursuit of the functions of the Wnt family of developmental regulators: insights from Xenopus laevis. Bioessays. 1993; 15:91-97. [PubMed: 8471061]

388. Moon RT, Brown JD, Torres M. WNTs modulate cell fate and behavior during vertebrate development. Trends Genet. 1997; 13:157-162. [PubMed: 9097727]

389. Moon RT, Campbell RM, Christian JL, McGrew LL, Shih J, Fraser S. Xwnt-5A: a maternal Wnt that affects morphogenetic movements after overexpression in embryos of Xenopus laevis. Development. 1993; 119:97-111. [PubMed: 8275867]

390. Moore JC, Sumerel JL, Schnackenberg BJ, Nichols JA, Wikramanayake A, Wessel GM, Marzluff WF. Cyclin D and cdk4 are required for normal development beyond the blastula stage in sea urchin embryos. Mol Cell Biol. 2002; 22:4863-4875. [PubMed: 12052892]

391. Moreau M, Leclerc C, Guanlandris-Parisot L, Duprat AM. Increased internal Ca ${ }^{2+}$ mediates neural induction in the amphibian embryo. Proc Natl Acad Sci U S A. 1994; 91:12639-12643. [PubMed: 7809092]

392. Moses RM, Kline D. Release of mouse eggs from metaphase arrest by protein synthesis inhibition in the absence of a calcium signal or microtubule assembly. Mol Reprod Dev. 1995; 41:264-273. [PubMed: 7654380] 
393. Moy GW, Kopf GS, Gache C, Vacquier VD. Calcium-mediated release of glucanase activity from cortical granules of sea urchin eggs. Dev Biol. 1983; 100:267-274. [PubMed: 6686171]

394. Muslin AJ, Peters KG, Williams LT. Direct activation of phospholipase $\mathrm{C} \gamma$ by fibroblast growth factor receptor is not required for mesoderm induction in Xenopus animal caps. Mol Cell Biol. 1994; 14:3006-3012. [PubMed: 8164656]

395. Muto A, Kume S, Inoue T, Okano H, Mikoshiba K. Calcium waves along the cleavage furrows in cleavage-stage Xenopus embryos and its inhibition by heparin. J Cell Biol. 1996; 135:181-190. [PubMed: 8858172]

396. Muto A, Mikoshiba K. Activation of inositol 1,4,5-trisphosphate receptors induces transient changes in cell shape of fertilized Xenopus eggs. Cell Motil Cytoskeleton. 1998; 39:201-208. [PubMed: 9519901]

397. Nakano Y, Shirakawa H, Mitsuhashi N, Kuwabara Y, Miyazaki S. Spatiotemporal dynamics of intracellular calcium in the mouse egg injected with a spermatozoon. Mol Hum Reprod. 1997; 3:1087-1093. [PubMed: 9464853]

398. Nauli, Surya M.; Alenghat, Francis J.; Luo, Y.; Williams, E.; Vassilev, P.; Li, X.; Elia, Andrew EH.; Lu, W.; Brown, Edward M.; Quinn, Stephen J.; Ingber , Donald E.; Zhou, J.; Ingber D E Harvard Med Sch BMA. Polycystins 1 and 2 mediate mechanosensation in the primary cilium of kidney cells. Nature genetics. 2003; 33:129-137. [PubMed: 12514735]

399. Ninomiya H, Elinson RP, Winklbauer R. Antero-posterior polarity links mesoderm convergent extension to axial patterning. Nature. 2004; 430:364-367. [PubMed: 15254540]

400. Nishinakamura R, Matsumoto Y, Uochi T, Asashima M, Yokota T. Xenopus FK 506-binding protein homolog induces a secondary axis in frog embryos, which is inhibited by coexisting BMP 4 signalling. Biochem Biophys Res Commun. 1997; 239:585-591. [PubMed: 9344875]

401. Nixon VL, Levasseur M, McDougall A, Jones KT. Ca ${ }^{2+}$ oscillations promote APC/C-dependent cyclin $\mathrm{B} 1$ degradation during metaphase arrest and completion of meiosis in fertilizing mouse eggs. Curr Biol. 2002; 12:746-750. [PubMed: 12007419]

402. Nixon VL, McDougall A, Jones KT. $\mathrm{Ca}^{2+}$ oscillations and the cell cycle at fertilisation of mammalian and ascidian eggs. Biol Cell. 2000; 92:187-196. [PubMed: 11043407]

403. Noguchi T, Mabuchi I. Localized calcium signals along the cleavage furrow of the Xenopus egg are not involved in cytokinesis. Mol Biol Cell. 2002; 13:1263-1273. [PubMed: 11950937]

404. Nonaka S, Shiratori S, Saijoh Y, Hamada H. Determination of left right patterning in the mouse embryo by artificial nodal flow. Nature. 2002; 418:96-99. [PubMed: 12097914]

405. Nonaka S, Tanaka Y, Okada Y, Takeda S, Harada A, Kanai Y, Kido M, Hitokawa N. Randomization of left-right asymmetry due to loss of nodal cilia generating leftward flow of extraembryonic fluid in mice kacking KIF3B motor protein. Cell. 1998; 95:829-837. [PubMed: 9865700]

406. Norris, Jeffrey W.; Tomczak, Melanie M.; Oliver, Ann E.; Tsvetkova, Nelly M.; Crowe, John H.; Tablin, F.; Nuccitelli, R. Structural Characterization of the ADAM 16 Disintegrin Loop Active Site. Biochemistry. 2003; 42:9813-9821. [PubMed: 12911325]

407. Nuccitelli R, Yim DL, Smart T. The sperm-induced $\mathrm{Ca}^{2+}$ wave following fertilization of the Xenopus egg requires the production of $\operatorname{Ins}(1,4,5) \mathrm{P}_{3}$. Dev Biol. 1993; 158:200-212. [PubMed: 7687224]

408. Nusco GA, Lim D, Sabala P, Santella L. $\mathrm{Ca}^{2+}$ response to cADPr during maturation and fertilization of starfish oocytes. Biochem Biophys Res Commun. 2002; 290:1015-1021. [PubMed: 11798176]

409. Nusslein-Volhard C. Of flies and fishes. Science. 1994; 266:572-574. [PubMed: 7939708]

410. Oda S, Deguchi R, Mohri T, Shikano T, Nakanishi S, Miyazaki S. Spatiotemporal dynamics of the $\left[\mathrm{Ca}^{2+}\right]_{\mathrm{i}}$ rise induced by microinjection of sperm extract into mouse eggs: preferential induction of a $\mathrm{Ca}^{2+}$ wave from the cortex mediated by the inositol 1,4,5-trisphosphate receptor. Dev Biol. 1999; 209:172-185. [PubMed: 10208751]

411. Okumura E, Fukuhara T, Yoshida H, Hanada Si S-i, Kozutsumi R, Mori M, Tachibana K, Kishimoto T. Akt inhibits Myt1 in the signalling pathway that leads to meiotic G2/M-phase transition. Nat Cell Biol. 2002; 4:111-116. [PubMed: 11802161] 
412. Osawa M, Takemoto K, Kikuyama M, Uchiyama H, Hiramoto Y, Kuroda H. Sperm and its soluble extract cause transient increases in intracellular calcium concentration and in membrane potential of sea urchin zygotes. Dev Biol. 1994; 166:268-276. [PubMed: 7958451]

413. Otte A, Moon RT. Protein kinase C isozymes have distinct roles in neural induction and neural competence in Xenopus. Cell. 1992; 68:1021-1029. [PubMed: 1547501]

414. Otte A, van Run P, Heidevelt M, van Driel R, Durston AJ. Neural induction is mediated by cross talk between the protein kinase C and cyclic AMP pathways. Cell. 1989; 58:641-648. [PubMed: 2548730]

415. Otte AP, Kramer IM, Mannesse M, Lambrechts C, Durston AJ. Characterization of protein kinase C in early Xenopus embryogenesis. Development. 1990; 110:461-470. [PubMed: 2133549]

416. Ozil JP, Huneau D. Activation of rabbit oocytes: the impact of the $\mathrm{Ca}^{2+}$ signal regime on development. Development. 2001; 128:917-928. [PubMed: 11222146]

417. Ozil JP, Swann K. Stimulation of repetitive calcium transients in mouse eggs. J Physiol. 1995; 483:331-346. [PubMed: 7650607]

418. Palermo GD, Avrech OM, Colombero LT, Wu H, Wolny YM, Fissore RA, Rosenwaks Z. Human sperm cytosolic factor triggers $\mathrm{Ca}^{2+}$ oscillations and overcomes activation failure of mammalian oocytes. Mol Hum Reprod. 1997; 3:367-374. [PubMed: 9237265]

419. Palma V, Kukulyan M, Mayor R. Calcium mediates dorsoventral patterning of mesoderm in Xenopus. Curr Biol. 2001; 11:1606-1610. [PubMed: 11676922]

420. Parrington J, Brind S, De Smedt H, Gangeswaran R, Lai FA, Wojcikiewicz R, Carroll J. Expression of inositol 1,4,5-trisphosphate receptors in mouse oocytes and early embryos: the type I isoform is upregulated in oocytes and downregulated after fertilization. Dev Biol. 1998; 203:451-461. [PubMed: 9808793]

421. Parrington J, Swann K, Shevchenko VI, Sesay AK, Lai FA. Calcium oscillations in mammalian eggs triggered by a soluble sperm protein. Nature. 1996; 379:364-368. [PubMed: 8552195]

422. Parry HD, McDougall AD, Whitaker M. Microdomains bounded by endoplasmic reticulum segregate cell cycle calcium transients in syncytial Drosophila embryos. J Cell Biol. 2005 accepted pending revision.

423. Patel R, Holt M, Philipova R, Moss S, Schulman H, Hidaka H, Whitaker M. Calcium/calmodulindependent phosphorylation and activation of human Cdc25-C at the G2/M phase transition in HeLa cells. J Biol Chem. 1999; 274:7958-7968. [PubMed: 10075693]

424. Pennekamp P, Karcher C, Fischer A, Schweickert A, Skryabin B, Horst J, Blum M, Dworniczak B. The ion channel polycystin-2 is required for left-right axis determination in mice. Curr Biol. 2002; 12:938-943. [PubMed: 12062060]

425. Perez-Terzic CM, Chini EN, Shen SS, Dousa TP, Clapham DE. Ca ${ }^{2+}$ release triggered by nicotinate adenine dinucleotide phosphate in intact sea urchin eggs. Biochem J. 1995; 312:955959. [PubMed: 8554544]

426. Perry G, Epel D. Characterization of a $\mathrm{Ca}^{2+}$-stimulated lipid peroxidizing system in the sea urchin egg. Dev Biol. 1985; 107:47-57. [PubMed: 3917415]

427. Perry G, Epel D. Fertilization stimulates lipid peroxidation in the sea urchin egg. Dev Biol. 1985; 107:58-65. [PubMed: 3917416]

428. Pesty A, Lefevre B, Kubiak J, Geraud G, Tesarik J, Maro B. Mouse oocyte maturation is affected by lithium via the polyphosphoinositide metabolism and the microtubule network. Mol Reprod Dev. 1994; 38:187-199. [PubMed: 8080648]

429. Philipova R, Larman M, Leckie C, Harrison P, Groigno L, Parry H, Whitaker M. MAP kinase activity regulates calcium release and mitosis entry in early sea urchin embryos. J Biol Chem. 2005; 280:24957-24967. [PubMed: 15843380]

430. Picard A, Giraud F, Le Bouffant F, Sladeczek F, Le Peuch C, Doree M. Inositol 1,4,5triphosphate microinjection triggers activation, but not meiotic maturation in amphibian and starfish oocytes. FEBS Lett. 1985; 182:446-450. [PubMed: 3920074]

431. Picard A, Karsenti E, Dabauvalle MC, Doree M. Release of mature starfish oocytes from interphase arrest by microinjection of human centrosomes. Nature. 1987; 327:170-172. [PubMed: 3106826] 
432. Piotrowska K, Zernicka-Goetz M. Role for sperm in spatial patterning of the early mouse embryo. Nature. 2001; 409:517-521. [PubMed: 11206548]

433. Plusa B, Hadjantonakis A-K, Gray D, Piotrowska-Nitsche K, Jedrusik A, Virginia E Papaioannou, David M Glover, Zernicka-Goetz M. The first cleavage of the mouse zygote predicts the blastocyst axis. Nature. 2005; 434:391-395. [PubMed: 15772664]

434. Poenie M, Alderton J, Steinhardt R, Tsien R. Calcium rises abruptly and briefly throughout the cell at the onset of anaphase. Science. 1986; 233:886-889. [PubMed: 3755550]

435. Polzonetti V, Cardinali M, Mosconi G, Natalini P, Meiri I, Carnevali O. Cyclic ADPR and calcium signaling in sea bream (Sparus aurata) egg fertilization. Mol Reprod Dev. 2002; 61:213217. [PubMed: 11803557]

436. Praetorius HA, Spring KR. Bending the primary cilium increases intracellular calcium. J Membr Biol. 2001; 184:71-79. [PubMed: 11687880]

437. Quill TA, Hedrick JL. The fertilization layer mediated block to polyspermy in Xenopus laevis: isolation of the cortical granule lectin ligand. Arch Biochem Biophys. 1996; 333:326-332. [PubMed: 8809070]

438. Rappaport R. Cleavage furrow establishment by the moving mitotic apparatus. Dev Growth Differ. 1997; 39:221-226. [PubMed: 9108335]

439. Raya A, Kawakami Y, Rodriguez-Esteban C, Buscher D, Koth CM, Itoh T, Morita M, Raya RM, Dubova I, Bessa JG, de la Pompa JL, Belmonte JC Izpizua. Notch activity induces Nodal expression and mediates the establishment of left-right asymmetry in vertebrate embryos. Genes Dev. 2003; 17:1213-1218. [PubMed: 12730123]

440. Raya A, Kawakami Y, Rodriguez-Esteban C, Ibanes M, Rasskin-Gutman D, Rodriguez-Leon J, Buscher D, Feijo JA, Izpisua Belmonte JC. Notch activity acts as a sensor for extracellular calcium during vertebrate left-right determination. Nature. 2004; 427:121-128. [PubMed: 14712268]

441. Reimann JDR, Jackson PK. Emi1 is required for cytostatic factor arrest in vertebrate eggs. Nature. 2002; 416:850-854. [PubMed: 11976684]

442. Reinhard E, Yokoe H, Niebling KR, Allbritton NL, Kuhn MA, Meyer T. Localized calcium signals in early zebrafish development. Dev Biol. 1995; 170:50-61. [PubMed: 7541377]

443. Rice A, Parrington J, Jones KT, Swann K. Mammalian sperm contain a $\mathrm{Ca}^{2+}$-sensitive phospholipase $\mathrm{C}$ activity that can generate $\mathrm{InsP}_{3}$ from $\mathrm{PIP}_{2}$ associated with intracellular organelles. Dev Biol. 2000; 228:125-135. [PubMed: 11087632]

444. Rizzuto R, Duchen Michael R, Pozzan T. Flirting in little space: the ER/mitochondria $\mathrm{Ca}^{2+}$ liaison. Sci STKE. 2004; 2004:re1. [PubMed: 14722345]

445. Robinson KR, Messerli M. The role of endogenous electrical fields as directional signals in development, repair and invasion. Bioessays. 2003; 25:759-766. [PubMed: 12879446]

446. Roderick HL, Lechleiter JD, Camacho P. Cytosolic phosphorylation of calnexin controls intracellular $\mathrm{Ca}^{2+}$ oscillations via an interaction with SERCA2b. J Cell Biol. 2000; 149:12351248. [PubMed: 10851021]

447. Roegiers F, McDougall A, Sardet C. The sperm entry point defines the orientation of the calciuminduced contraction wave that directs the first phase of cytoplasmic reorganization in the ascidian egg. Development. 1995; 121:3457-3466. [PubMed: 7588078]

448. Rongish BJ, Kinsey WH. Transient nuclear localization of Fyn kinase during development in zebrafish. Anat Rec. 2000; 260:115-123. [PubMed: 10993948]

449. Rongish BJ, Wu W, Kinsey WH. Fertilization-induced activation of phospholipase C in the sea urchin egg. Dev Biol. 1999; 215:147-154. [PubMed: 10545226]

450. Rossignol DP, Decker GL, Lennarz WJ, Tsong TY, Teissie J. Induction of calcium-dependent, localized cortical granule breakdown in sea-urchin eggs by voltage pulsation. Biochim Biophys Acta. 1983; 763:346-355. [PubMed: 6652114]

451. Runft LL, Jaffe LA. Sperm extract injection into ascidian eggs signals $\mathrm{Ca}^{2+}$ release by the same pathway as fertilization. Development. 2000; 127:3227-3236. [PubMed: 10887079]

452. Runft LL, Jaffe LA, Mehlmann LM. Egg activation at fertilization: where it all begins. Dev Biol. 2002; 245:237-254. [PubMed: 11977978] 
453. Runft LL, Watras J, Jaffe LA. Calcium release at fertilization of Xenopus eggs requires type $\mathrm{I} \mathrm{IP}_{3}$ receptors, but not SH2 domain-mediated activation of PLC $\gamma$ or $\mathrm{G}_{\mathrm{q}}$-mediated activation of PLC $\beta$. Dev Biol. 1999; 214:399-411. [PubMed: 10525343]

454. Ryan PJ, Gillespie LL. Phosphorylation of phospholipase $\mathrm{C} \gamma 1$ and its association with the FGF receptor is developmentally regulated and occurs during mesoderm induction in Xenopus leavis. Dev Biol. 1994; 166:101-111. [PubMed: 7958437]

455. Sadler KC, Ruderman JV. Components of the signalling pathway linking the 1-methyladenine receptor to MPF activation and maturation in starfish oocytes. Dev Biol. 1998; 197:25-38. [PubMed: 9578616]

456. Samuel AD, Murthy VN, Hengartner MO. Calcium dynamics during fertilization in C. elegans. BMC Dev Biol. 2001; 1:8. [PubMed: 11346453]

457. Saneyoshi T, Kume S, Amasaki Y, Mikoshiba K. The Wnt/calcium pathway activates NF-AT and promotes ventral cell fate in Xenopus embryos. Nature. 2002; 417:295-299. [PubMed: 12015605]

458. Santella L, De Riso L, Gragnaniello G, Kyozuka K. Cortical granule translocation during maturation of starfish oocytes requires cytoskeletal rearrangement triggered by $\mathrm{InsP}_{3}$-mediated $\mathrm{Ca}^{2+}$ release. Exp Cell Res. 1999; 248:567-574. [PubMed: 10222148]

459. Santella L, De Riso L, Gragnaniello G, Kyozuka K. Separate activation of the cytoplasmic and nuclear calcium pools in maturing starfish oocytes. Biochem Biophys Res Commun. 1998; 252:1-4. [PubMed: 9813135]

460. Santella L, Ercolano E, Lim D, Nusco GA, Moccia F. Activated M-phase-promoting factor (MPF) is exported from the nucleus of starfish oocytes to increase the sensitivity of the Ins(1,4,5) $\mathrm{P}_{3}$ receptors. Biochemical Society transactions. 2003; 31:79-82. [PubMed: 12546658]

461. Santella L, Kyozuka K. Association of calmodulin with nuclear structures in starfish oocytes and its role in the resumption of meiosis. Eur J Biochem. 1997; 246:602-610. [PubMed: 9219515]

462. Santella L, Kyozuka K. Effects of 1-methyladenine on nuclear $\mathrm{Ca}^{2+}$ transients and meiosis resumption in starfish oocytes are mimicked by the nuclear injection of inositol 1,4,5trisphosphate and cADP-ribose. Cell Calcium. 1997; 22:11-20. [PubMed: 9232348]

463. Santella L, Kyozuka K. Reinitiation of meiosis in starfish oocytes requires an increase in nuclear $\mathrm{Ca}^{2+}$ Biochem Biophys Res Commun. 1994; 203:674-680. [PubMed: 8074721]

464. Santella L, Kyozuka K, Genazzani AA, De Riso L, Carafoli E. Nicotinic acid adenine dinucleotide phosphate-induced $\mathrm{Ca}^{2+}$ release. Interactions among distinct $\mathrm{Ca}^{2+}$ mobilizing mechanisms in starfish oocytes. J Biol Chem. 2000; 275:8301-8306. [PubMed: 10722659]

465. Santella L, Lim D, Moccia F. Calcium and fertilization: the beginning of life. Trends Biochem Sci. 2004; 29:400-408. [PubMed: 15362223]

466. Sasai Y, De Robertis EM. Ectodermal patterning in vertebrate embryos. Dev Biol. 1997; 182:520. [PubMed: 9073437]

467. Sasaki H, Epel D. Cortical vesicle exocytosis in isolated cortices of sea urchin eggs: description of a turbidometric assay and its utilization in studying effects of different media on discharge. Dev Biol. 1983; 98:327-337. [PubMed: 6683684]

468. Sater A, Alderton JM, Steinhardt RA. An increase in intracellular pH during neural induction in Xenopus. Development. 1994; 120:433-442. [PubMed: 8149919]

469. Sato K, Iwasaki T, Hirahara S, Nishihira Y, Fukami Y. Molecular dissection of egg fertilization signaling with the aid of tyrosine kinase-specific inhibitor and activator strategies. Biochim Biophys Acta. 2004; 1697:103-121. [PubMed: 15023354]

470. Sato K, Iwasaki T, Ogawa K, Konishi M, Alexander A Tokmakov, Fukami Y. Low density detergent-insoluble membrane of Xenopus eggs: subcellular microdomain for tyrosine kinase signaling in fertilization. Development. 2002; 129:885-896. [PubMed: 11861472]

471. Sato K, Tokmakov AA, Iwasaki T, Fukami Y. Tyrosine kinase-dependent activation of phospholipase $\mathrm{C} \gamma$ is required for calcium transient in Xenopus egg fertilization. Dev Biol. 2000; 224:453-469. [PubMed: 10926780]

472. Sato MS, Yoshitomo M, Mohri T, Miyazaki S. Spatiotemporal analysis of $\left[\mathrm{Ca}^{2+}\right]_{i}$ rises in mouse eggs after intracytoplasmic sperm injection (ICSI). Cell Calcium. 1999; 26:49-58. [PubMed: 10892570] 
473. Sato Y, Miyazaki S, Shikano T, Mitsuhashi N, Takeuchi H, Mikoshiba K, Kuwabara Y. Adenophostin, a potent agonist of the inositol 1,4,5-trisphosphate receptor, is useful for fertilization of mouse oocytes injected with round spermatids leading to normal offspring. Biol Reprod. 1998; 58:867-873. [PubMed: 9510978]

474. Saunders CM, Larman MG, Parrington J, Cox LJ, Royse J, Blayney LM, Swann K, Lai FA. PLC zeta: a sperm-specific trigger of $\mathrm{Ca}^{2+}$ oscillations in eggs and embryo development. Development. 2002; 129:3533-3544. [PubMed: 12117804]

475. Schatten G. The centrosome and its mode of inheritance: the reduction of the centrosome during gametogenesis and its restoration during fertilization. Dev Biol. 1994; 165:299-335. [PubMed: 7958403]

476. Schatten H, Hueser CN, Chakrabarti A. From fertilization to cancer: the role of centrosomes in the union and separation of genomic material. Microsc Res Tech. 2000; 49:420-427. [PubMed: 10842368]

477. Schmidt T, Patton C, Epel D. Is there a role for the $\mathrm{Ca}^{2+}$ influx during fertilization of the sea urchin egg? Dev Biol. 1982; 90:284-290. [PubMed: 7075862]

478. Schnackenberg BJ, Marzluff WF. Novel localization and possible functions of cyclin E in early sea urchin development. J Cell Sci. 2002; 115:113-121. [PubMed: 11801729]

479. Sconzo G, Cascino D, Amore G, Geraci F, Giudice G. Effect of the IMPase inhibitor L690,330 on sea urchin development. Cell Biol Int. 1998; 22:91-94. [PubMed: 9878095]

480. SeGall GK, Lennarz WJ. Jelly coat and induction of the acrosome reaction in echinoid sperm. Dev Biol. 1981; 86:87-93. [PubMed: 7286401]

481. Shapiro BM. Molecular aspects of sperm-egg fusion. Ciba Found Symp. 1984

482. Shearer J, De Nadai C, Emily-Fenouil F, Gache C, Whitaker M, Ciapa B. Role of phospholipase $\mathrm{C} \gamma$ at fertilization and during mitosis in sea urchin eggs and embryos. Development. 1999; 126:2273-2284. [PubMed: 10207151]

483. Sheldahl LC. Protein kinase C is differentially stimulated by Wnt and Frizzled homologs in a Gprotein-dependent manner. Curr Biol. 1999; 9:695-698. [PubMed: 10395542]

484. Sheldahl LC, Slusarski Diane C, Pandur P, Miller Jeffrey R, Kühl M, Moon Randall T. Dishevelled activates $\mathrm{Ca}^{2+}$ flux, PKC, and CaMKII in vertebrate embryos. J Cell Biol. 2003; 161:769-777. [PubMed: 12771126]

485. Shen SS, Buck WR. Sources of calcium in sea urchin eggs during the fertilization response. Dev Biol. 1993; 157:157-169. [PubMed: 8482408]

486. Shen SS, Kinsey WH, Lee SJ. Protein tyrosine kinase-dependent release of intracellular calcium in the sea urchin egg. Dev Growth Differ. 1999; 41:345-355. [PubMed: 10400396]

487. Shen SS, Steinhardt RA. Intracellular $\mathrm{pH}$ and the sodium requirement at fertilisation. Nature. 1979; 282:87-89. [PubMed: 41185]

488. Shilling F, Chiba K, Hoshi M, Kishimoto T. Pertussis toxin inhibits 1-methyladenine-induced maturation in starfish oocytes. Dev Biol. 1989; 133:605-608. [PubMed: 2499496]

489. Shilling F, Mandel G, Jaffe LA. Activation by serotonin of starfish eggs expressing the rat serotonin 1c receptor. Cell Regul. 1990; 1:465-469. [PubMed: 1981686]

490. Shimoda Y, Kitajima K, Inoue S, Inoue Y. Isolation, structural determination, and calciumbinding properties of the major glycoprotein present in Bufo japonicus japonicus egg jelly. Eur J Biochem. 1994; 223:223-231. [PubMed: 8033895]

491. Shiwa M, Murayama T, Ogawa Y. Molecular cloning and characterization of ryanodine receptor from unfertilized sea urchin eggs. Am J Physiol. 2002; 282:R727-737.

492. Shuster CB, Burgess DR. Targeted new membrane addition in the cleavage furrow is a late, separate event in cytokinesis. Proc Natl Acad Sci U S A. 2002; 99:3633-3638. [PubMed: 11891298]

493. Shuster CB, Burgess DR. Transitions regulating the timing of cytokinesis in embryonic cells. Curr Biol. 2002; 12:854-858. [PubMed: 12015124]

494. Simon JZ, Cooper MS. Calcium oscillations and calcium waves coordinate rhythmic contractile activity within the stellate cell layer of medaka fish embryos. J Exp Zool. 1995; 273:118-129. 
495. Slusarski DC, Corces VG, Moon RT. Interaction of Wnt and a frizzled homologue triggers Gprotein-linked phosphatidylinositol signalling. Nature. 1997; 390:410-413. [PubMed: 9389482]

496. Slusarski DC, YangSnyder J, Busa WB, Moon RT. Modulation of embryonic intracellular $\mathrm{Ca}^{2+}$ signaling by Wnt-5A. Dev Biol. 1997; 182:114-120. [PubMed: 9073455]

497. Smith JC, Slack JMW. Dorsalization and neural induction: properties of the organizer in Xenopus laevis. J Embryol Exp Morphol. 1983; 78:299-317. [PubMed: 6663230]

498. Smith WC, Harland RM. Injected $X$ wnt- 8 RNA acts early in Xenopus embryos to promote formation of a dorsalizing centre. Cell. 1991; 67:753-765. [PubMed: 1657405]

499. Smith WC, McKendry R, Ribisi S Jr, Harland RM. A nodal-related gene defines a physical and functional domain within the Spemann organizer. Cell. 1995; 82:37-46. [PubMed: 7606783]

500. Sokol S, Christian JL, Moon RT, Melton DA. Injected Wnt RNA induces a complete body axis in Xenopus. Cell. 1991; 67:741-752. [PubMed: 1834344]

501. Solovyova N, Veselovsky N, Toescu EC, Verkhratsky A. $\mathrm{Ca}^{2+}$ dynamics in the lumen of the endoplasmic reticulum in sensory neurons: direct visualization of $\mathrm{Ca}^{2+}$-induced $\mathrm{Ca}^{2+}$ release triggered by physiological $\mathrm{Ca}^{2+}$ entry. EMBO J. 2002; 21:622-630. [PubMed: 11847110]

502. Speksnijder JE. The repetitive calcium waves in the fertilized ascidian egg are initiated near the vegetal pole by a cortical pacemaker. Dev Biol. 1992; 153:259-271. [PubMed: 1397683]

503. Speksnijder JE, Corson DW, Sardet C, Jaffe LF. Free calcium pulses following fertilization in the ascidian egg. Dev Biol. 1989; 135:182-190. [PubMed: 2767333]

504. Speksnijder JE, Sardet C, Jaffe LF. The activation wave of calcium in the ascidian egg and its role in ooplasmic segregation. J Cell Biol. 1990; 110:1589-1598. [PubMed: 2335565]

505. Speksnijder JE, Sardet C, Jaffe LF. Periodic calcium waves cross ascidian eggs after fertilization. Dev Biol. 1990; 142:246-249. [PubMed: 2227098]

506. Speksnijder JE, Terasaki M, Hage WJ, Jaffe LF, Sardet C. Polarity and reorganization of the endoplasmic reticulum during fertilization and ooplasmic segregation in the ascidian egg. J Cell Biol. 1993; 120:1337-1346. [PubMed: 8449980]

507. Squirrell, JM.; Ji, J.; Walker, JW.; White, JG. Unreveling the role of calcium signalling in the early C. elegans embryo. 2001. http://elegansswmededu/wli/[wm2001p731] (cited with permission)

508. Stayner C, Zhou J. Polycystin channels and kidney disease. Trends Pharmacol Sci. 2001; 22:543546. [PubMed: 11698076]

509. Steinhardt R, Zucker R, Schatten G. Intracellular calcium release at fertilization in the sea urchin egg. Dev Biol. 1977; 58:185-196. [PubMed: 326602]

510. Steinhardt RA, Alderton J. Intracellular free calcium rise triggers nuclear envelope breakdown in the sea urchin embryo. Nature. 1988; 332:364-366. [PubMed: 3127727]

511. Steinhardt RA, Bi G, Alderton JM. Cell-membrane resealing by a vesicular mechanism similar to neurotransmitter release. Science. 1994; 263:390-393. [PubMed: 7904084]

512. Steinhardt RA, Epel D. Activation of sea-urchin eggs by a calcium ionophore. Proc Natl Acad Sci U S A. 1974; 71:1915-1919. [PubMed: 4525301]

513. Steinhardt RA, Epel D, Carroll EJ, Yanagimachi R. Is calcium ionophore a universal activator for unfertilised eggs? Nature. 1974; 252:41-43. [PubMed: 4473722]

514. Steinhardt RA, Lundin L, Mazia D. Bioelectric responses of the echinoderm egg to fertilization. Proc Natl Acad Sci U S A. 1971; 68:2426-2430. [PubMed: 5289876]

515. Steinhardt RA, Morita H, Hodgson ES. Mode of action of straight chain hydrocarbons on primary chemoreceptors of the blowfly, Phormia regina. J Cell Physiol. 1966; 67:53-62. [PubMed: 5937017]

516. Stephano JL, Gould MC. The intracellular calcium increase at fertilization in Urechis caupo oocytes: activation without waves. Dev Biol. 1997; 191:53-68. [PubMed: 9356171]

517. Stith BJ, Espinoza R, Roberts D, Smart T. Sperm increase inositol 1,4,5-trisphosphate mass in Xenopus laevis eggs preinjected with calcium buffers or heparin. Dev Biol. 1994; 165:206-215. [PubMed: 8088439] 
518. Stith BJ, Goalstone M, Silva S, Jaynes C. Inositol 1,4,5-trisphosphate mass changes from fertilization through first cleavage in Xenopus laevis. Mol Biol Cell. 1993; 4:435-443. [PubMed: 8507898]

519. Strachan T, Abitbol M, Davidson D, Beckmann JS. A new dimension for the human genome project: towards comprehensive expression maps. Nat Genet. 1997; 16:126-132. [PubMed: 9171823]

520. Streb H, Irvine RF, Berridge MJ, Schulz I. Release of $\mathrm{Ca}^{2+}$ from a nonmitochondrial intracellular store in pancreatic acinar-cells by inositol-1,4,5-trisphosphate. Nature. 1983; 306:67-69. [PubMed: 6605482]

521. Streuli C. Extracellular matrix remodelling and cellular differentiation. Curr Opin Cell Biol. 1999; 11:634-640. [PubMed: 10508658]

522. Stricker SA. Comparative biology of calcium signaling during fertilization and egg activation in animals. Dev Biol. 1999; 211:157-176. [PubMed: 10395780]

523. Stricker SA. Intracellular injections of a soluble sperm factor trigger calcium oscillations and meiotic maturation in unfertilized oocytes of a marine worm. Dev Biol. 1997; 186:185-201. [PubMed: 9205139]

524. Stricker SA. Repetitive calcium waves induced by fertilization in the nemertean worm Cerebratulus lacteus. Dev Biol. 1996; 176:243-263. [PubMed: 8660865]

525. Stricker SA. Time-lapse confocal imaging of calcium dynamics in starfish embryos. Dev Biol. 1995; 170:496-518. [PubMed: 7649379]

526. Stricker SA, Centonze VE, Melendez RF. Calcium dynamics during starfish oocyte maturation and fertilization. Dev Biol. 1994; 166:34-58. [PubMed: 7958457]

527. Stricker SA, Centonze VE, Paddock SW, Schatten G. Confocal microscopy of fertilizationinduced calcium dynamics in sea urchin eggs. Dev Biol. 1992; 149:370-380. [PubMed: 1730391]

528. Stricker SA, Silva R, Smythe T. Calcium and endoplasmic reticulum dynamics during oocyte maturation and fertilization in the marine worm Cerebratulus lacteus. Dev Biol. 1998; 203:305322. [PubMed: 9808782]

529. Stricker SA, Swann K, Jones KT, Fissore RA. Injections of porcine sperm extracts trigger fertilization-like calcium oscillations in oocytes of a marine worm. Exp Cell Res. 2000; 257:341347. [PubMed: 10837148]

530. Stricker SAS, T.L. Endoplasmic reticulum reorganizations and $\mathrm{Ca}^{2+}$ signaling in maturing and fertilized oocytes of marine protostome worms: the roles of MAPKs and MPF. Development. 2003; 130:2867-2879. [PubMed: 12756171]

531. Sumerel JL, Moore JC, Schnackenberg BJ, Nichols JA, Canman JC, Wessel GM, Marzluff WF. Cyclin E and its associated cdk activity do not cycle during early embryogenesis of the sea Urchin. Dev Biol. 2001; 234:425-440. [PubMed: 11397011]

532. Supp DM, Witte DP, Potter SS, Brueckner M. Mutation of an axonemal dynein affects left-right asymmetry in inversus viscerum mice. Nature. 1997; 389:963-966. [PubMed: 9353118]

533. Swann K. A cytosolic sperm factor stimulates repetitive calcium increases and mimics fertilization in hamster eggs. Development. 1990; 110:1295-1302. [PubMed: 2100264]

534. Swann K. Different triggers for calcium oscillations in mouse eggs involve a ryanodine-sensitive calcium store. Biochem J. 1992; 287:79-84. [PubMed: 1417794]

535. Swann K. Soluble sperm factors and $\mathrm{Ca}^{2+}$ release in eggs at fertilization. Rev Reprod. 1996; 1:33-39. [PubMed: 9414436]

536. Swann K, Igusa Y, Miyazaki S. Evidence for an inhibitory effect of protein kinase C on Gprotein-mediated repetitive calcium transients in hamster eggs. EMBO J. 1989; 8:3711-3718. [PubMed: 2510999]

537. Swann K, McCulloh DH, McDougall A, Chambers EL, Whitaker M. Sperm-induced currents at fertilization in sea urchin eggs injected with EGTA and neomycin. Dev Biol. 1992; 151:552-563. [PubMed: 1318235]

538. Swann K, McDougall A, Whitaker M. Calcium signalling at fertilization. J Mar Biolog Assoc U K. 1994; 74:3-16. 
539. Swann K, Parrington J. Mechanism of $\mathrm{Ca}^{2+}$ release at fertilization in mammals. J Exp Zool. 1999; 285:267-275. [PubMed: 10497326]

540. Swann K, Whitaker M. The part played by inositol trisphosphate and calcium in the propagation of the fertilization wave in sea urchin eggs. J Cell Biol. 1986; 103:2333-2342. [PubMed: 3491080]

541. Swann K, Whitaker M. Stimulation of the $\mathrm{Na} / \mathrm{H}$ exchanger of sea urchin eggs by phorbol ester. Nature. 1985; 314:274-277. [PubMed: 2984569]

542. Swanson WJ, Vacquier VD. Concerted evolution in an egg receptor for a rapidly evolving abalone sperm protein. Science. 1998; 281:710-712. [PubMed: 9685267]

543. Swezey RR, Epel D. Regulation of glucose-6-phosphate dehydrogenase activity in sea urchin eggs by reversible association with cell structural elements. J Cell Biol. 1986; 103:1509-1515. [PubMed: 3771646]

544. Tabin CJ, Vogan KJ. A two-cilia model for vertebrate left-right axis specification. Genes Dev. 2003; 17:1-6. [PubMed: 12514094]

545. Tada M, Smith JC. Xwnt 11 is a target of Xenopus Brachyury: regulation of gastrulation movements via Dishevelled, but not through the canonical Wnt pathway. Development. 2000; 127:2227-2238. [PubMed: 10769246]

546. Tahara M, Tasaka K, Masumoto N, Mammoto A, Ikebuchi Y, Miyake A. Dynamics of cortical granule exocytosis at fertilization in living mouse eggs. Am J Physiol. 1996; 270:C1354-1361. [PubMed: 8967435]

547. Takahashi T, Saito H, Hiroi M, Doi K, Takahashi E. Effects of aging on inositol 1,4,5triphosphate-induced $\mathrm{Ca}^{2+}$ release in unfertilized mouse oocytes. Mol Reprod Dev. 2000; 55:299-306. [PubMed: 10657049]

548. Takeuchi H, Oike M, Paterson HF, Allen V, Kanematsu T, Ito Y, Erneux C, Katan M, Hirata M. Inhibition of $\mathrm{Ca}^{2+}$ signalling by p130, a phospholipase-C-related catalytically inactive protein: critical role of the p130 pleckstrin homology domain. Biochem J. 2000; 349:357-368. [PubMed: 10861248]

549. Talmor-Cohen A, Tomashov-Matar R, Eliyahu E, Shapiro R, Shalgi R. Are Src family kinases involved in cell cycle resumption in rat eggs? Reproduction. 2004; 127:455-463. [PubMed: 15047936]

550. Tanaka Y, Okada Y, Hirokawa N. FGF-induced vesicular release of Sonic hedgehog and retinoic acid in leftward nodal flow in critical for left-right determination. Nature. 2005; 435:172-177. [PubMed: 15889083]

551. Tang TS, Dong JB, Huang XY, Sun FZ. $\mathrm{Ca}^{2+}$ oscillations induced by a cytosolic sperm protein factor are mediated by a maternal machinery that functions only once in mammalian eggs. Development. 2000; 127:1141-1150. [PubMed: 10662652]

552. Terasaki M, Jaffe LA. Organization of the sea urchin egg endoplasmic reticulum and its reorganization at fertilization. J Cell Biol. 1991; 114:929-940. [PubMed: 1874789]

553. Terasaki M, Jaffe LA, Hunnicutt GR, Hammer JA. Structural change of the endoplasmic reticulum during fertilization: evidence for loss of membrane continuity using the green fluorescent protein. Dev Biol. 1996; 179:320-328. [PubMed: 8903348]

554. Terasaki M, Okumura E-I, Hinkle B, Kishimoto T. Localization and dynamics of Cdc2-cyclin B during meiotic reinitiation in starfish oocytes. Mol Biol Cell. 2003; 14:4685-4694. [PubMed: 14551249]

555. Terasaki M, Runft LL, Hand AR. Changes in organization of the endoplasmic reticulum during Xenopus oocyte maturation and activation. Mol Biol Cell. 2001; 12:1103-1116. [PubMed: 11294910]

556. Terasaki M, Song J, Wong JR, Weiss MJ, Chen LB. Localization of endoplasmic reticulum in living and glutaraldehyde-fixed cells with fluorescent dyes. Cell. 1984; 38:101-108. [PubMed: 6432338]

557. Thaler CD, Kuo RC, Patton C, Preston CM, Yagisawa H, Epel D. Phosphoinositide metabolism at fertilization of sea urchin eggs measured with a GFP-probe. Develop Growth Differ. 2004; 46:413-423. 
558. Thomas JM, Masgrau R, Churchill GC, Galione A. Pharmacological characterization of the putative cADP-ribose receptor. Biochem J. 2001; 359:451-457. [PubMed: 11583594]

559. Thomas TW, Eckberg WR, Dube F, Galione A. Mechanisms of calcium release and sequestration in eggs of Chaetopterus pergamentaceus. Cell Calcium. 1998; 24:285-292. [PubMed: 9883282]

560. Thorn P, Gerasimenko O, Petersen OH. Cyclic ADP-ribose regulation of ryanodine receptors involved in agonist evoked cytosolic $\mathrm{Ca}^{2+}$ oscillations in pancreatic acinar cells. EMBO J. 1994; 13:2038-2043. [PubMed: 7514529]

561. Togo T, Alderton JM, Bi GQ, Steinhardt RA. The mechanism of facilitated cell membrane resealing. J Cell Sci. 1999; 112:719-731. [PubMed: 9973606]

562. Togo T, Alderton JM, Steinhardt RA. The mechanism of cell membrane repair. Zygote. 2000; 8:S31-32. [PubMed: 11191298]

563. Tokmakov AA, Sato KI, Iwasaki T, Fukami Y. Src kinase induces calcium release in Xenopus egg extracts via PLC $\gamma$ and IP $_{3}$-dependent mechanism. Cell Calcium. 2002; 32:11-20. [PubMed: 12127058]

564. Tombes RM, Simerly C, Borisy GG, Schatten G. Meiosis, egg activation, and nuclear envelope breakdown are differentially reliant on $\mathrm{Ca}^{2+}$, whereas germinal vesicle breakdown is $\mathrm{Ca}^{2+}$ independent in the mouse oocyte. J Cell Biol. 1992; 117:799-811. [PubMed: 1577859]

565. Toratani S, Katada T, Yokosawa H. Botulinum ADP-ribosyltransferase C3 induces elevation of the vitelline coat of ascidian eggs. Biochem Biophys Res Commun. 1993; 193:1311-1317. [PubMed: 8323551]

566. Török K, Wilding M, Groigno L, Patel R, Whitaker M. Imaging the spatial dynamics of calmodulin activation during mitosis. Curr Biol. 1998; 8:692-699. [PubMed: 9637920]

567. Torres M, Yang-Snyder JA, Purcell SM, DeMarais AA, McGrew LL, Moon RT. Activities of the Wnt 1 class of secreted signaling factots are antagonized by the Wnt-5A class and by a dominant negative cadherin in early Xenopus development. J Cell Biol. 1996; 133:1123-1137. [PubMed: 8655584]

568. Tosti E, Dale B. Regulation of the fertilization current in ascidian oocytes by intracellular second messengers. Mol Reprod Dev. 1994; 37:473-476. [PubMed: 8011333]

569. Tunquist BJ, Schwab MS, Chen LG, Maller JL. The spindle checkpoint kinase bub1 and cyclin e/ cdk2 both contribute to the establishment of meiotic metaphase arrest by cytostatic factor. Curr Biol. 2002; 12:1027-1033. [PubMed: 12123578]

570. Turner E, Somers CE, Shapiro BM. The relationship between a novel NAD(P)H oxidase activity of ovoperoxidase and the $\mathrm{CN}$-resistant respiratory burst that follows fertilization of sea urchin eggs. J Biol Chem. 1985; 260:13163-13171. [PubMed: 4055735]

571. Turner PR, Jaffe LA, Fein A. Regulation of cortical vesicle exocytosis in sea urchin eggs by inositol 1,4,5-trisphosphate and GTP-binding protein. J Cell Biol. 1986; 102:70-76. [PubMed: 3001104]

572. Turner PR, Sheetz MP, Jaffe LA. Fertilization increases the polyphosphoinositide content of sea urchin eggs. Nature. 1984; 310:414-415. [PubMed: 6087155]

573. Twigg J, Patel R, Whitaker M. Translational control of $\mathrm{InsP}_{3}$-induced chromatin condensation during the early cell cycles of sea urchin embryos. Nature. 1988; 332:366-369. [PubMed: 3127728]

574. Umbhauer M, Penzo-Mendez A, Clavilier L, Boucaut J, Riou J. Signaling specificities of fibroblast growth factor receptors in early Xenopus embryos. J Cell Sci. 2000; 113:2865-2875. [PubMed: 10910771]

575. Vacquier VD. Evolution of gamete recognition proteins. Science. 1998; 281:1995-1998. [PubMed: 9748153]

576. Van der Wal J, Habets R, Varnai P, Balla T, Jalink K. Monitoring agonist-induced phospholipase $\mathrm{C}$ activation in live cells by fluorescence resonance energy transfer. J Biol Chem. 2001; 276:15337-15344. [PubMed: 11152673]

577. Vanderzwalmen P, Zech H, Birkenfeld A, Yemini M, Bertin G, Lejeune B, Nijs M, Segal L, Stecher A, Vandamme B, van Roosendaal E, Schoysman R. Intracytoplasmic injection of spermatids retrieved from testicular tissue: influence of testicular pathology, type of selected spermatids and oocyte activation. Hum Reprod. 1997; 12:1203-1213. [PubMed: 9222002] 
578. Veeman MT, Slusarski DC, Kaykas A, Hallagan LS, Moon RT. Zebrafish prickle, a modulator of noncanonical Wnt/Fz signaling, regulates gastrulation movements. Curr Biol. 2003; 13:680-685. [PubMed: 12699626]

579. Verlhac MH, Kubiak JZ, Clarke HJ, Maro B. Microtubule and chromatin behavior follow MAP kinase activity but not MPF activity during meiosis in mouse oocytes. Development. 1994; 120:1017-1025. [PubMed: 7600950]

580. Verlhac MH, Kubiak JZ, Weber M, Geraud G, Colledge WH, Evans MJ, Maro B. Mos is required for MAP kinase activation and is involved in microtubule organization during meiotic maturation in the mouse. Development. 1996; 122:815-822. [PubMed: 8631259]

581. Viets LN, Campbell KD, White KL. Pathways involved in RGD-mediated calcium transients in mature bovine oocytes. Cloning Stem Cells. 2001; 3:105-113. [PubMed: 11945220]

582. Vincent C, Cheek TR, Johnson MH. Cell cycle progression of parthenogenetically activated mouse oocytes to interphase is dependent on the level of internal calcium. J Cell Sci. 1992; 103:389-396. [PubMed: 1478942]

583. Wakabayashi S, Sardet C, Fafournoux P, Counillon L, Meloche S, Pages G, Pouyssegur J. Structure function of the growth factor-activatable $\mathrm{Na}^{+} / \mathrm{H}^{+}$exchanger (NHE1). Rev Physiol Biochem Pharmacol. 1992; 119:157-186. [PubMed: 1318573]

584. Walker DS, Gower NJD, Ly S, Bradley GL, Baylis HA. Regulated disruption of inositol 1,4,5trisphosphate signalling in Caenorhabditis elegans reveals new functions in feeding and embryogenesis. Mol Biol Cell. 2002; 13:1329-1337. [PubMed: 11950942]

585. Walker JW, Gilbert SH, Drummond RM, Yamada M, Sreekumar R, Carraway RE, Ikebe M, Fay FS. Signaling pathways underlying eosinophil cell motility revealed by using caged peptides. Proc Natl Acad Sci U S A. 1998; 95:1568-1573. [PubMed: 9465056]

586. Wallingford JB, Ewald AJ, Harland RM, Fraser SE. Calcium signaling during convergent extension in Xenopus. Curr Biol. 2001; 11:652-661. [PubMed: 11369228]

587. Wallingford JB, Harland RM. Xenopus Dishevelled signaling regulates both neural and mesodermal convergent extension: parallel forces elongating the body axis. Development. 2001; 128:2581-2592. [PubMed: 11493574]

588. Wallingford JB, Rowning BA, Vogeli KM, Rothbächer U, Fraser SE, Harland RM. Dishevelled controls cell polarity during Xenopus gastrulation. Nature. 2000; 405:81-85. [PubMed: 10811222]

589. Wang FS, Bonder EM. Sea urchin egg villin: identification of villin in a non-epithelial cell from an invertebrate species. J Cell Sci. 1991; 100:61-71. [PubMed: 1795031]

590. Wang S, Luo Y, Wilson Patricia D, Witman George, Zhou J. The autosomal recessive polycystic kidney disease protein is localized to primary cilia, with concentration in the basal body area. $\mathrm{J}$ Am Soc Nephrol. 2004; 15:592-602. [PubMed: 14978161]

591. Wang T, Li B-Y, Danielson PD, Shah PC, Rockwell S, Lechleider RJ, Martin J, Manganaro T, Donahoe PK. The immunophilin FKBP12 functions as a common inhibitor of the TGF $\beta$ Family Type I receptors. Cell. 1996; 86:435-444. [PubMed: 8756725]

592. Wassarman PM. Fertilization in animals. Dev Genet. 1999; 25:83-86. [PubMed: 10440841]

593. Webb DJ, Nuccitelli R. Fertilization potential and electrical properties of the Xenopus laevis egg. Dev Biol. 1985; 107:395-406. [PubMed: 3972161]

594. Webb SE, Lee KW, Karplus E, Miller AL. Localized calcium transients accompany furrow positioning, propagation, and deepening during the early cleavage period of zebrafish embryos. Dev Biol. 1997; 192:78-92. [PubMed: 9405098]

595. Webb SE, Miller AL. Calcium signalling during embryonic development. Nat Rev Mol Cell Biol. 2003; 4:539-551. [PubMed: 12838337]

596. Webb SE, Miller AL. Calcium signalling during zebrafish embryonic development. Bioessays. 2000; 22:113-123. [PubMed: 10655031]

597. Webb SE, Moreau M, Leclerc C, Miller AL. Calcium in development: from ion transients to gene expression. Bioessays. 2001; 23:372-374. [PubMed: 11268044]

598. Webb TA, Fluck RA. Microfilament- and microtubule-based movements during ooplasmic segregation in the medaka fish egg. Mol Biol Cell. 1993; 4:274a. 
599. Westfall TA, Brimeyer R, Twedt J, Gladon J, Olberding A, Furutani-Seiki M, Slusarski DC. Wnt $5 /$ pipetail functions in vertebrate axis formation as a negative regulator of $\mathrm{Wnt} / \beta$-catenin activity. J Cell Biol. 2003; 162:889-898. [PubMed: 12952939]

600. Westfall TA, Hjertos B, Slusarski DC. Requirement for intracellular calcium modulation in zebrafish dorsal-ventral patterning. Dev Biol. 2003; 259:380-391. [PubMed: 12871708]

601. Whalley T, McDougall A, Crossley I, Swann K, Whitaker M. Internal calcium release and activation of sea urchin eggs by cGMP are independent of the phosphoinositide signaling pathway. Mol Biol Cell. 1992; 3:373-383. [PubMed: 1320962]

602. Whitaker M. Control of meiotic arrest. Rev Reprod. 1996; 1:127-135. [PubMed: 9414449]

603. Whitaker M, Swann K. Lighting the fuse at fertilization. Development. 1993; 117:1-12.

604. Whitaker M, Aitchison M. Calcium-dependent polyphosphoinositide hydrolysis is associated with exocytosis in vitro. FEBS Lett. 1985; 182:119-124. [PubMed: 2982669]

605. Whitaker M, Irvine RF. Inositol 1,4,5-trisphosphate microinjection activates sea urchin eggs. Nature. 1984; 312:636-639.

606. Whitaker MJ, Baker PF. Calcium-dependent exocytosis in an in vitro secretory granule plasma membrane preparation from sea urchin eggs and the effects of some inhibitors of cytoskeletal function. Proc R Soc Lond B Biol Sci. 1983; 218:397-413. [PubMed: 6136975]

607. Whitaker MJ, Steinhardt RA. Evidence in support of the hypothesis of an electrically mediated fast block to polyspermy in sea-urchin eggs. Dev Biol. 1983; 95:244-248. [PubMed: 6825929]

608. Whitaker MJ, Steinhardt RA. Ionic regulation of egg activation. Q Rev Biophys. 1982; 15:593666. [PubMed: 6820160]

609. Wilding M, Dale B. Soluble extracts from ascidian spermatozoa trigger intracellular calcium release independently of the activation of the ADP ribose channel. Zygote. 1998; 6:149-154. [PubMed: 9770780]

610. Wilding M, Dale B. Sperm factor: what is it and what does it do? Mol Hum Reprod. 1997; 3:269273. [PubMed: 9237253]

611. Wilding M, Russo GL, Galione A, Marino M, Dale B. ADP-ribose gates the fertilization channel in ascidian oocytes. Am J Physiol. 1998; 275:C1277-1283. [PubMed: 9814976]

612. Wilding M, Torok K, Whitaker M. Activation-dependent and activation-independent localisation of calmodulin to the mitotic apparatus during the first cell cycle of the Lytechinus pictus embryo. Zygote. 1995; 3:219-224. [PubMed: 8903791]

613. Wilding M, Wright EM, Patel R, Ellis-Davies G, Whitaker M. Local perinuclear calcium signals associated with mitosis-ENTRY in early sea urchin embryos. J Cell Biol. 1996; 135:191-200. [PubMed: 8858173]

614. Williams CJ, Mehlmann LM, Jaffe LA, Kopf GS, Schultz RM. Evidence that $\mathrm{G}_{\mathrm{q}}$ family G proteins do not function in mouse egg activation at fertilization. Dev Biol. 1998; 198:116-127. [PubMed: 9640335]

615. Williams CJ, Schultz RM, Kopf GS. Role of G proteins in mouse egg activation: stimulatory effects of acetylcholine on the ZP2 to ZP2f conversion and pronucelar formation in eggs expressing a functional m1 muscarinic receptor. Dev Biol. 1992; 151:288-296. [PubMed: 1577193]

616. Williams RM, Zipfel WR, Webb WW. Multiphoton microscopy in biological research. Curr Opin Chem Biol. 2001; 5:603-608. [PubMed: 11578936]

617. Willmott N, Sethi JK, Walseth TF, Lee HC, White AM, Galione A. Nitric oxide-induced mobilization of intracellular calcium via the cyclic ADP-ribose signaling pathway. J Biol Chem. 1996; 271:3699-3705. [PubMed: 8631983]

618. Winklbauer R, Medina A, Swain RK, Steinbeisser H. Frizzled-7 signalling controls tissue separation during Xenopus gastrulation. Nature. 2001; 413:856-860. [PubMed: 11677610]

619. Winkler MM, Steinhardt RA, Grainger JL, Minning L. Dual ionic controls for the activation of protein synthesis at fertilization. Nature. 1980; 287:558-560. [PubMed: 7422008]

620. Witchel HJ, Steinhardt RA. 1-Methyladenine can consistently induce a fura-detectable transient calcium increase which is neither necessary nor sufficient for maturation in oocytes of the starfish Asterina miniata. Dev Biol. 1990; 141:393-398. [PubMed: 2210042] 
621. Wojcikiewicz RJ, Furuichi T, Nakade S, Mikoshiba K, Nahorski SR. Muscarinic receptor activation down-regulates the type 1 inositol 1,4,5-trisphosphate receptor by accelerating its degradation. J Biol Chem. 269:7063-7969. 269.

622. Wolny YM, Fissore RA, Wu H, Reis MM, Colombero LT, Ergun B, Rosenwaks Z, Palermo GD. Human glucosamine-6-phosphate isomerase, a homologue of hamster oscillin, does not appear to be involved in $\mathrm{Ca}^{2+}$ release in mammalian oocytes. Mol Reprod Dev. 1999; 52:277-287. [PubMed: 10206659]

623. Woods ML, Shimizu Y. Signaling networks regulating $\beta 1$ integrin-mediated adhesion of $T$ lymphocytes to extracellular matrix. J Leukoc Biol. 2001; 69:874-880. [PubMed: 11404370]

624. Wu H, Smyth J, Luzzi V, Fukami K, Takenawa T, Black SL, Allbritton NL, Fissore RA. Sperm factor induces intracellular free calcium oscillations by stimulating the phosphoinositide pathway. Biol Reprod. 2001; 64:1338-1349. [PubMed: 11319137]

625. Wyrick RE, Nishihara T, Hedrick JL. Agglutination of jelly coat and cortical granule components and the block to polyspermy in the amphibian Xenopus laevis. Proc Natl Acad Sci U S A. 1974; 71:2067-2071. [PubMed: 4525317]

626. Xu XZS, Paul W Sternberg. A C. elegans sperm TRP protein required for sperm-egg interactions during fertilization. Cell. 2003; 114:285-297. [PubMed: 12914694]

627. Yamamoto S, Kubota HY, Yoshimoto Y, Iwao Y. Injection of a sperm extract triggers egg activation in the newt Cynops pyrrhogaster. Dev Biol. 2001; 230:89-99. [PubMed: 11161564]

628. Yang-Snyder J, Miller JR, Brown JD, Lai CJ, Moon RT. A frizzled homolog functions in a vertebrate Wnt signalling pathway. Curr Biol. 1996; 6:1302-1306. [PubMed: 8939578]

629. Yazaki I. $\mathrm{Ca}^{2+}$ in specification of vegetal cell fate in early sea urchin embryos. J Exp Biol. 2001; 204:823-834. [PubMed: 11171406]

630. Yazaki I, Abe M, Santella L, Koyama Y. Mechanisms of calcium elevation in the micromeres of sea urchin embryos. Biol Cell. 2004; 96:153-167. [PubMed: 15050370]

631. Yoda A, Oda S, Shikano T, Kouchi Z, Awaji T, Shirakawa H, Kinoshita K, Miyazaki S. Ca ${ }^{2+}$ oscillation-inducing phospholipase $\mathrm{C} \zeta$ expressed in mouse eggs is accumulated to the pronucleus during egg activation. Dev Biol. 2004; 268:245-257. [PubMed: 15063165]

632. Yoshida M, Sensui N, Inoue T, Morisawa M, Mikoshiba K. Role of two series of $\mathrm{Ca}^{2+}$ oscillations in activation of ascidian eggs. Dev Biol. 1998; 203:122-133. [PubMed: 9806778]

633. Yoshida Y, Kim S, Chiba K, Kawai S, Tachikawa H, Takahashi N. Calcineurin inhibitors block dorsal side signalling that affect late-stage development of the heart, kidney, liver, gut and somitic tissue during Xenopus embryogenesis. Dev Growth Differ. 2004; 46:139-152. [PubMed: 15066193]

634. Yoshigaki T. Simulation of the mechanism of determining the position of the cleavage furrow in cytokinesis of sea urchin eggs. Math Biosci. 2001; 170:17-58. [PubMed: 11259802]

635. Yoshimoto Y, Hiramoto Y. Observation of intracellular $\mathrm{Ca}^{2+}$ with aequorin luminescence. Int Rev Cytol. 1991; 129:45-73. [PubMed: 1917380]

636. Zernicka-Goetz M. Patterning of the embryo: the first spatial decisions in the life of a mouse. Development. 2002; 129:815-829. [PubMed: 11861466]

637. Zhu CC, Furuichi T, Mikoshiba K, Wojcikiewicz RJ. Inositol 1,4,5-trisphosphate receptor downregulation is activated directly by inositol 1.4.5-trisphosphate binding. J Biol Chem. 1999; 274:3476-3484. [PubMed: 9920893]

638. Zhu X, Evans JP. Analysis of the roles of RGD-binding integrins, a4/a9 integrins, a 6 integrins, and CD9 in the interaction of the fertilin $\beta$ (ADAM2) disintegrin domain with the mouse egg membrane. Biol Reprod. 2002; 66:1193-1202. [PubMed: 11906941]

639. Zucker RS, Steinhardt RA. Prevention of the cortical reaction in fertilized sea urchin eggs by injection of calcium-chelating ligands. Biochim Biophys Acta. 1978; 541:459-466. [PubMed: 96872] 


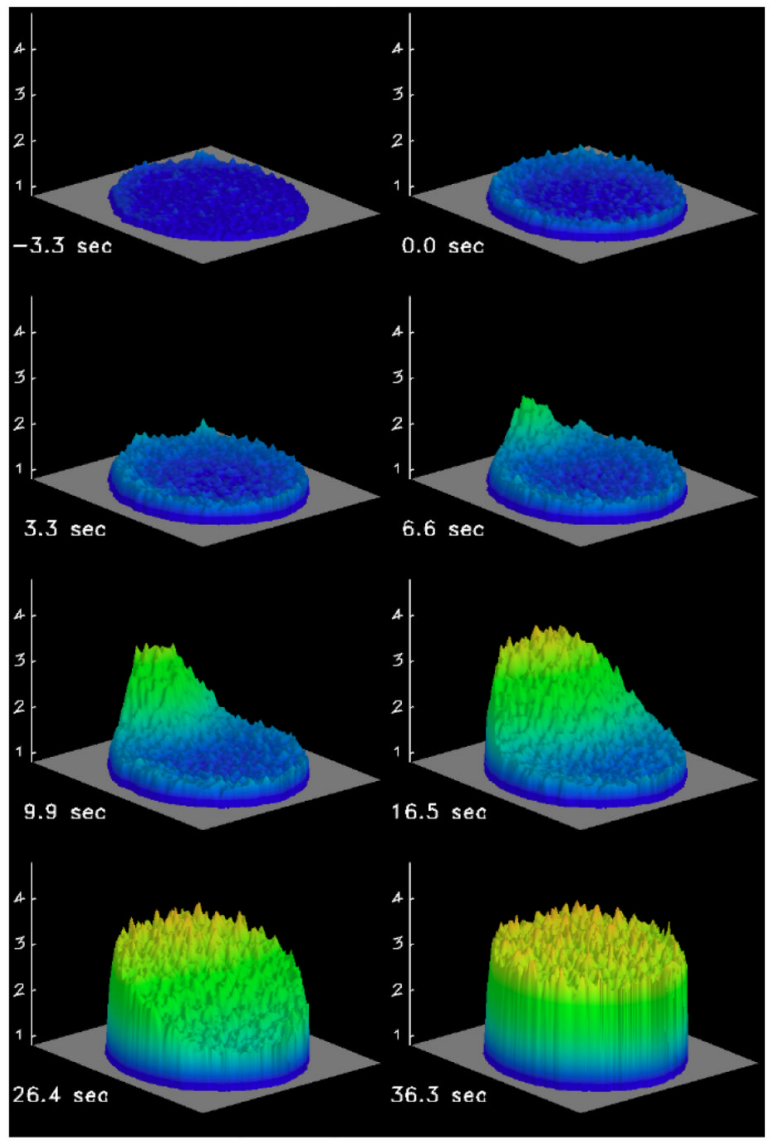

Figure 1.

The fertilization calcium wave in a sea urchin (Lytechinus pictus) egg.

The calcium wave initiates at the point of sperm entry and crosses the egg as a tsunami-like wave, traversing the egg in around 20s. Calcium concentrations were visualized using the calcium indicator calcium green dextran and confocal microscopy. Calcium levels are represented by warm colours and height in this topographical plot. A pseudoratio image is generated by pixelwise division of each image by an image of resting dye distribution acquired before fertilization. Adapted from reference 360 . 


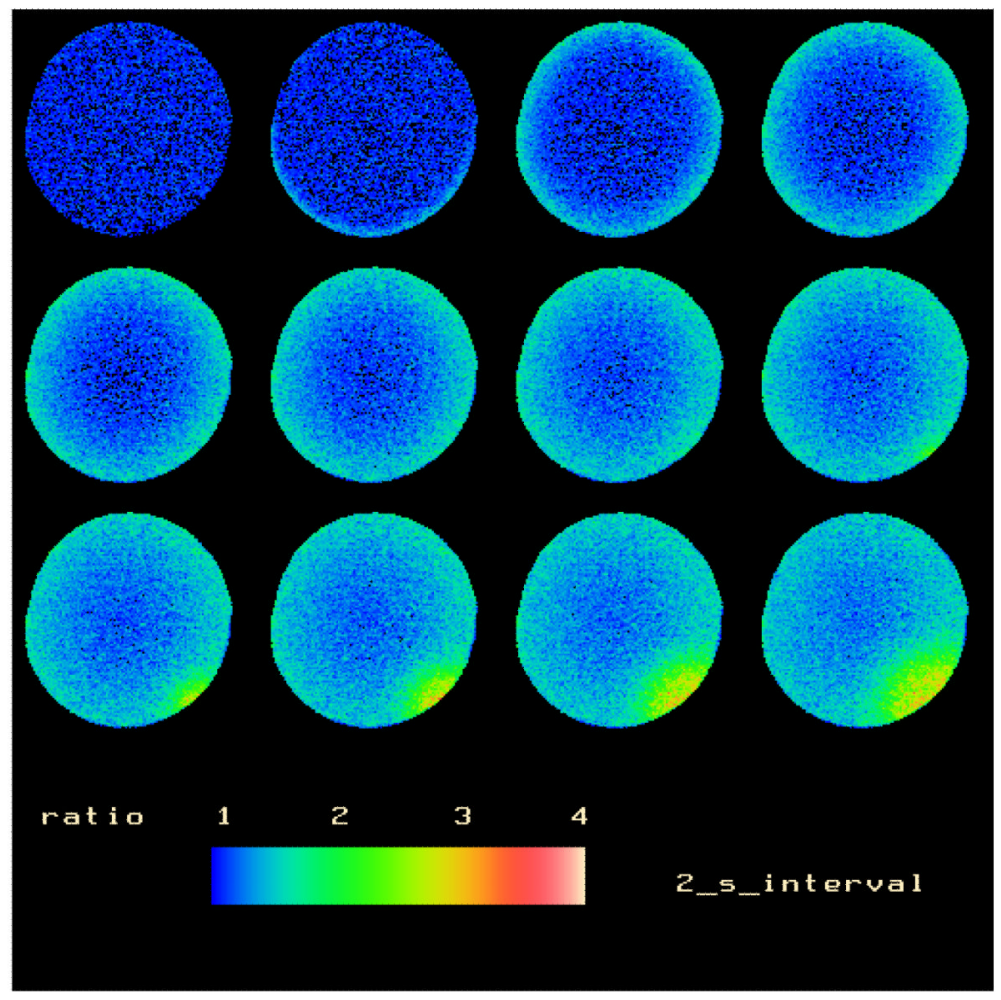

Figure 2.

The action potential and latent period at fertilization.

Relatively rapid confocal scanning microscopy reveals the action potential as a small elevation of calcium just beneath the plasma membrane. Images read as one reads text, from left to right and top to bottom. The second image shows the rapid influx: it occurs about halfway through the confocal scan, giving rise to an image that appears to show influx in only the bottom half of the image. Careful inspection will show that this subcortical calcium increase is also present in Figure 1 at the point of fertilization. The latent period is the time between the action potential and the initiation of the calcium wave in image 8 and is around $15 \mathrm{~s}$ long. During this period no change in cytoplasmic calcium concentration is detectable. Methods as for Figure 1. Originally published in reference 538. 


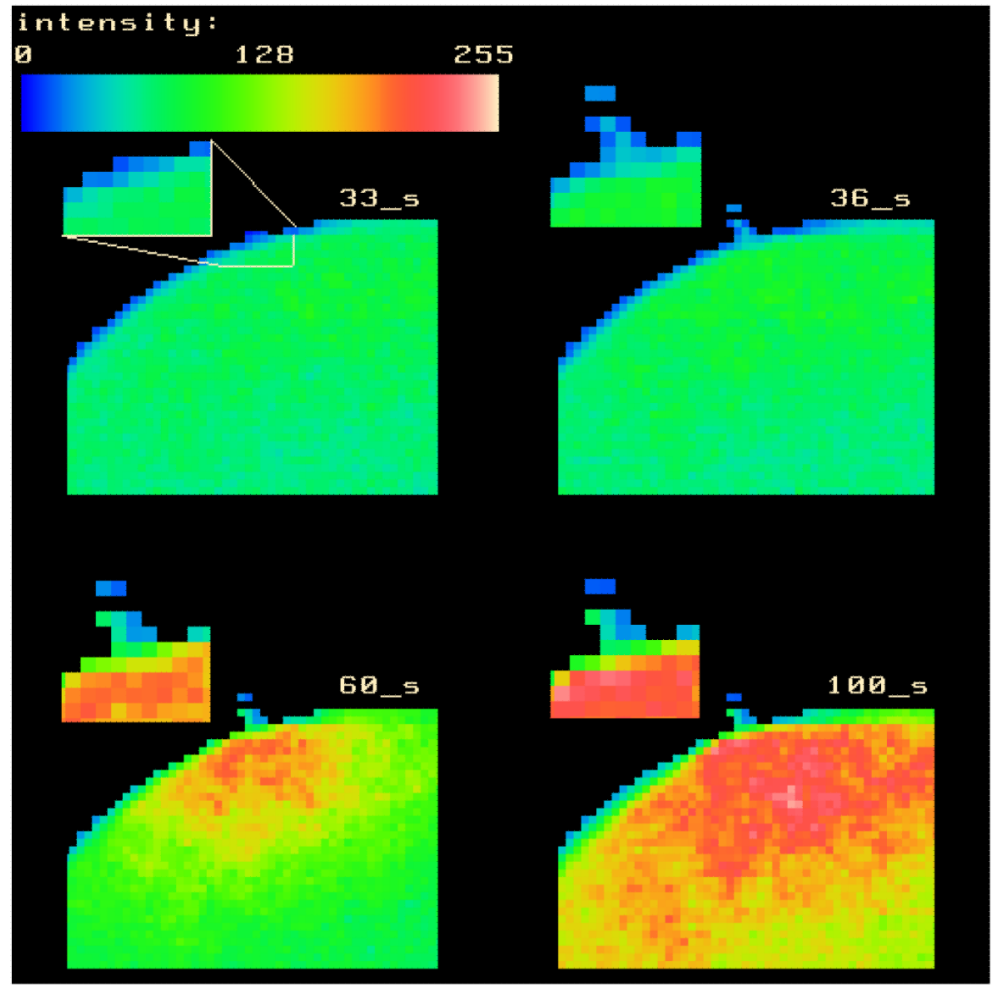

Figure 3.

Sperm egg fusion.

Sperm egg fusion can be detected through the transfer of calcium green dye from egg to sperm. The data demonstrate that sperm egg fusion occurs before calcium concentrations increase at the site of fusion and the calcium wave is initiated. Methods as for Figure 1. Adapted from reference 538 . 

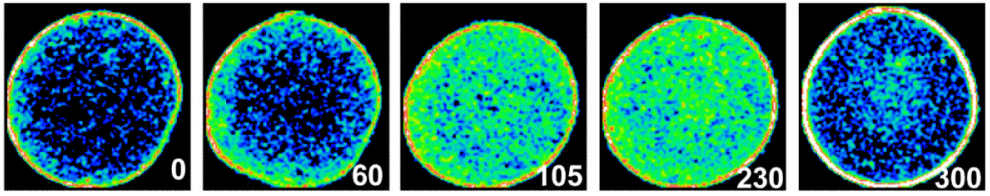

Figure 4.

InsP3 increases at fertilization.

Images of a Lytechinus pictus egg microinjected with a PLC $\delta$ PH domain-green fluorescent protein chimera. As InsP3 is generated at fertilization, it competes for binding of the probe with plasma membrane PtdInsP2, causing the fluorescent probe to leave the plasma membrane and accumulate in the cytoplasm. As InsP3 levels fall, the probe leaves the cytoplasm and reaccumulates on the plasma membrane. Note the slow time course of this response relative to the release of calcium: because the diffusion constant of the protein chimera is perhaps 50-fold lower than that of InsP3, it is not a faithful spatiotemporal indicator of InsP3 increase, but can be used to estimate the maximal InsP3 concentrations at fertilization. 


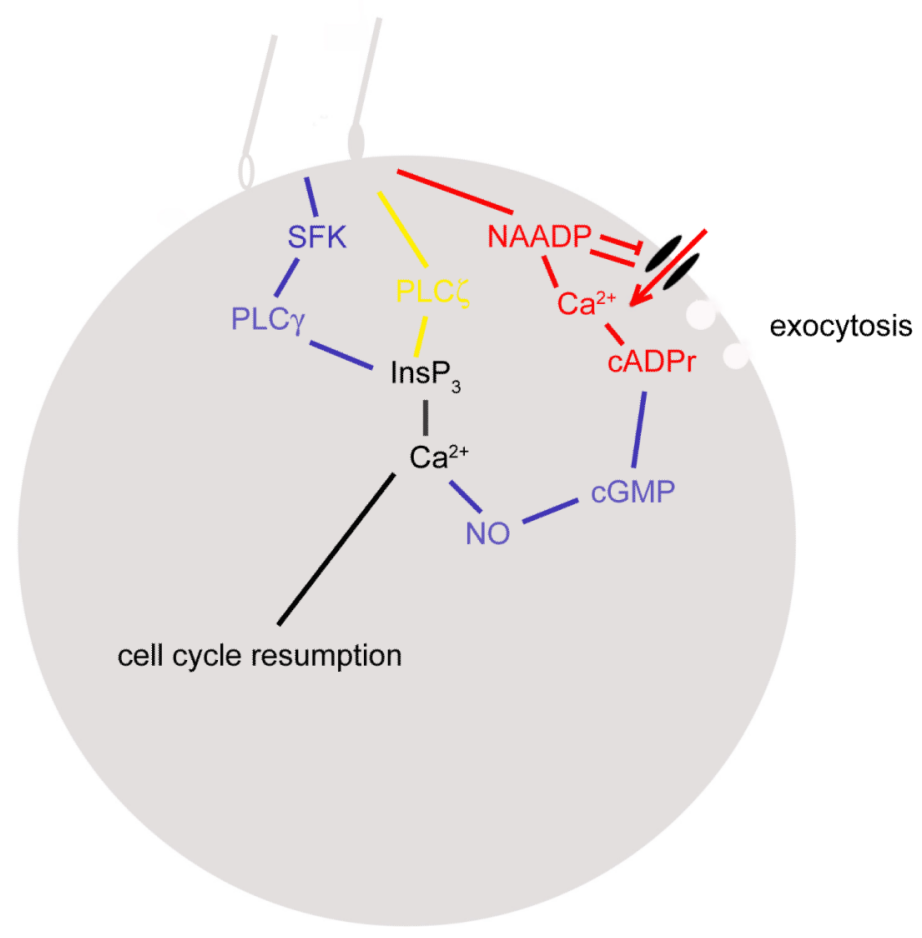

Figure 5.

Signalling pathways at fertilization.

In frog, ascidian and echinoderms, src family kinases (SFK) activate PLC $\gamma$ to produce $\mathrm{InsP}_{3}$ and trigger the calcium waves (blue pathway); in sea urchin, there is good evidence that sperm-egg fusion is required for egg activation, but in frog this is less certain. In sea urchin, calcium activates nitric oxide production which generates cADPr via cGMP (blue pathway). In mammals, sperm-egg fusion introduces PLC $\zeta$ into the egg cytoplasm, so producing InsP $_{3}$ (yellow pathway). Sperm egg fusion may also introduce NAADP in echinoderms; NAADP activates plasma membrane calcium channels (red pathway). In ascidian, NAADP inactivates plasma membrane channels, while cADPr triggers local calcium release to trigger cortical granule exocytosis (red pathway). 

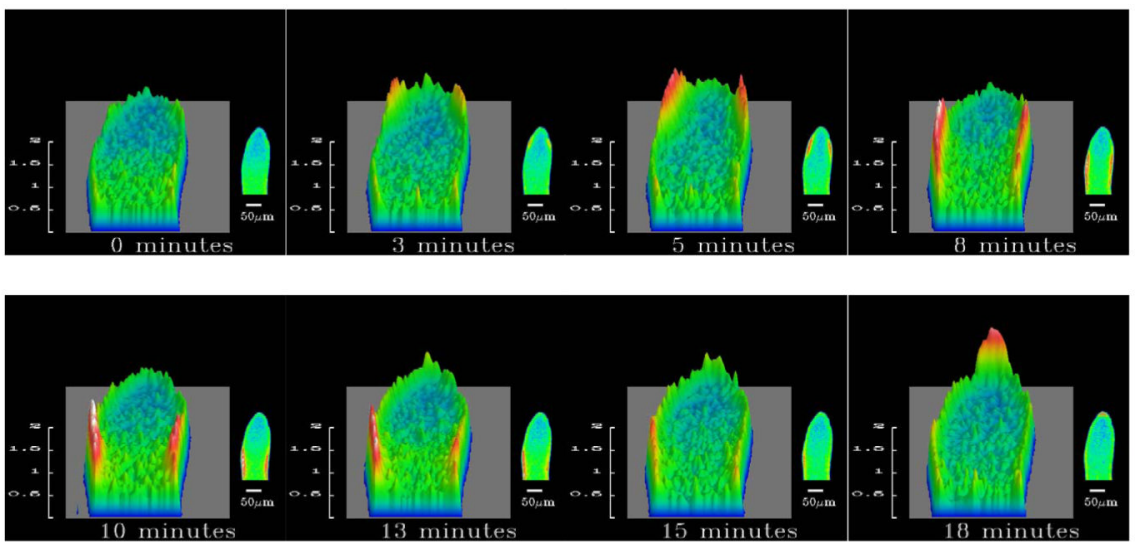

Figure 6.

Slow mitotic calcium waves in syncytial Drosophila embryos.

Calcium waves move from pole to equator in Drosophila embryos, in step with mitotic waves. The calcium waves are subcortical, as are the embryo nuclei (not visible); the centre of a Drosophila embryo is a yolky mass. Ratiometric imaging using calcium green- and rhodamine-dextran. The images are displayed topographically, with small non-topographical images for comparison. 

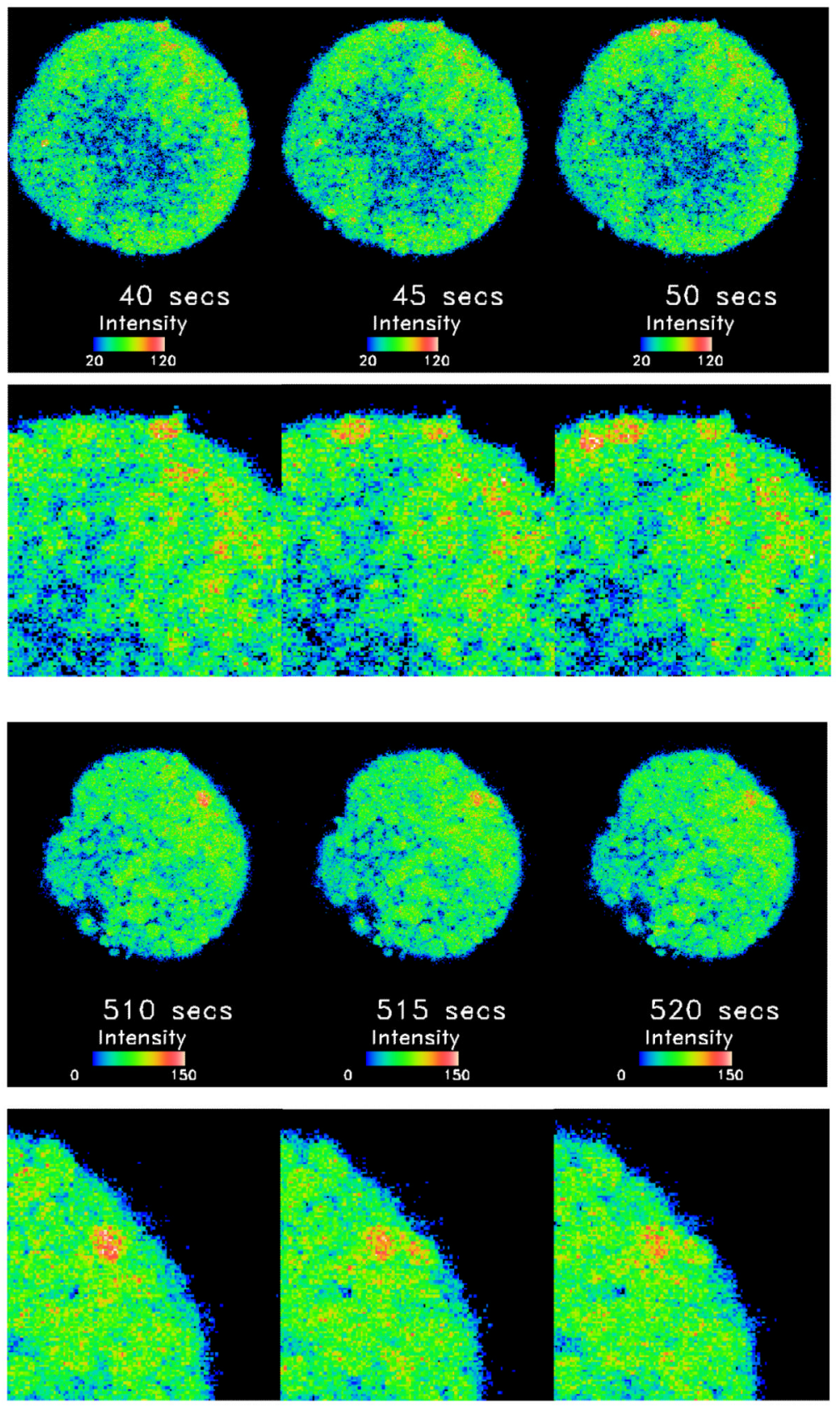

Figure 7.

Calcium signals in the enveloping layer of zebrafish (Dania rerio) embryos during the blastodisc stage.

Calcium transients occur sporadically in the enveloping layer. Two examples are shown of a common observation that transients are correlated in adjacent cells. Calcium green-/ rhodamine-dextran confocal ratio imaging of a mid-blastula blastodisc viewed en face. 

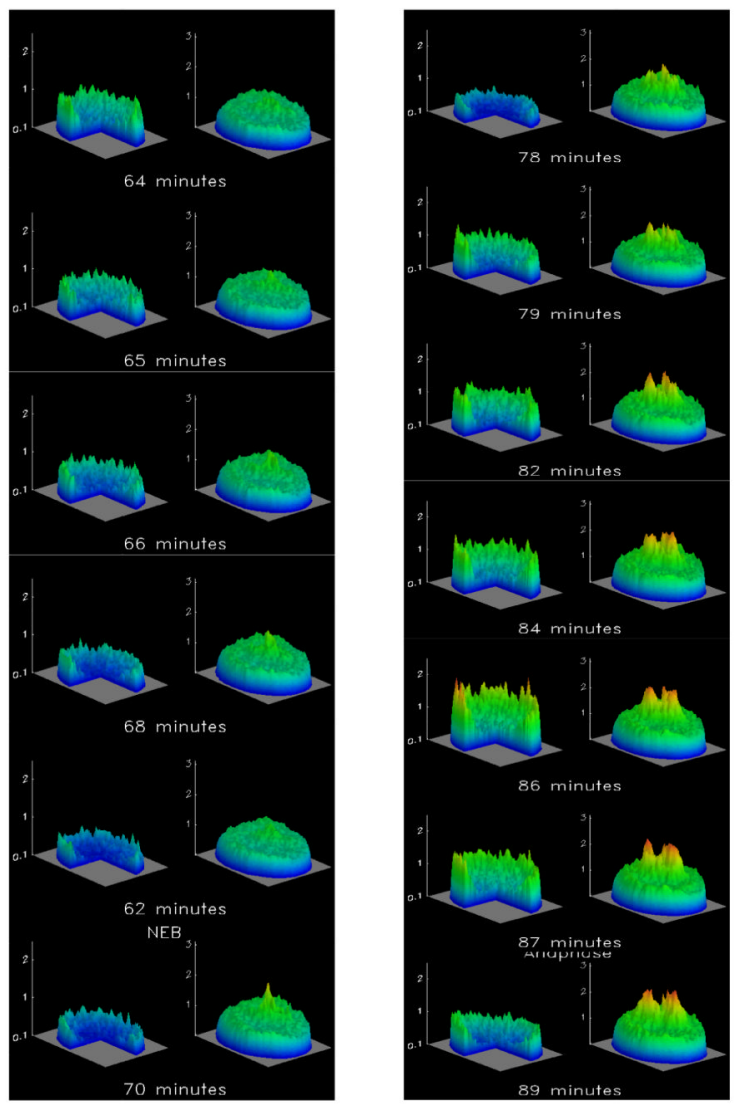

Figure 8.

Calcium and calmodulin imaging during mitosis in sea urchin (Lytechinus pictus) embryos. Left hand images show calcium concentrations during mitosis, represented topographically. Right hand images show active calmodulin. Calcium increases at around 64 minutes after fertilization, leading to a local activation of calmodulin in the perinuclear region at 70 minutes as NEB occurs. A second increase in calcium at $86 \mathrm{~min}$ is associated with activation of calmodulin at the mitotic spindle poles just before anaphase. Note that the spatiotemporal pattern of calmodulin activation is determined by recruitment of active calmodulin to its targets. Calcium concentrations were measured by ratiometric imaging with calcium greenand rhodamine-dextran. Active calmodulin was imaged by ratiometric imaging of TA- and fluorescein calmodulin. TA-calmodulin senses calmodulin activation and concentration, while fluorescein-calmodulin senses concentration alone. Adapted from references 188 and 566. 

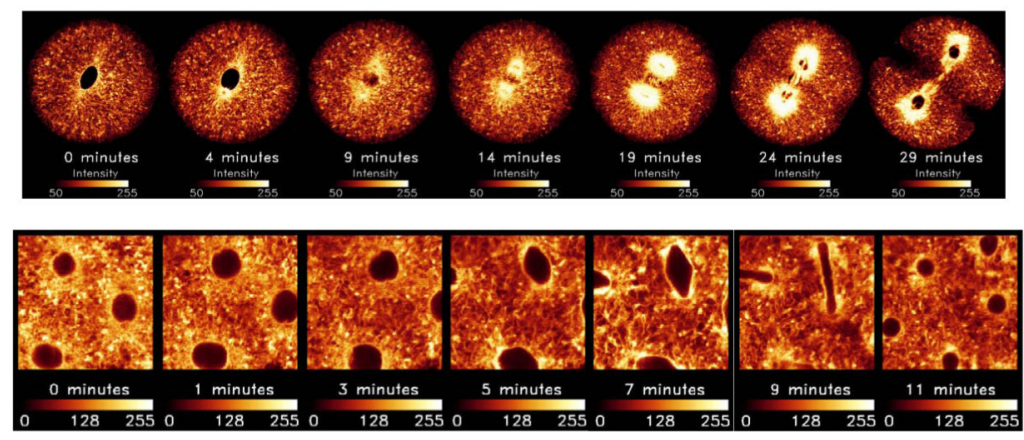

Figure 9.

Accumulation of endoplasmic reticulum around the nucleus and mitotic spindle during mitosis in sea urchin (Lytechinus pictus) and Drosophila embryos.

Upper panel, sea urchin. The ER begins to accumulate as the nucleus elongates after centrosome duplication in prophase; ER increasingly accumulates around the spindle poles, but not the chromosomes throughout metaphase and anaphase.

Lower panel, Drosophila. In these embryos, the spindle remains bounded by ER which does not enter the spindle. Nonetheless, ER accumulates around the ends of the spindle during metaphase and anaphase. In both cases, ER is visualized by injection of the lipophilic dye, $\operatorname{Di}(\mathrm{I}) \mathrm{C}_{16}$. 

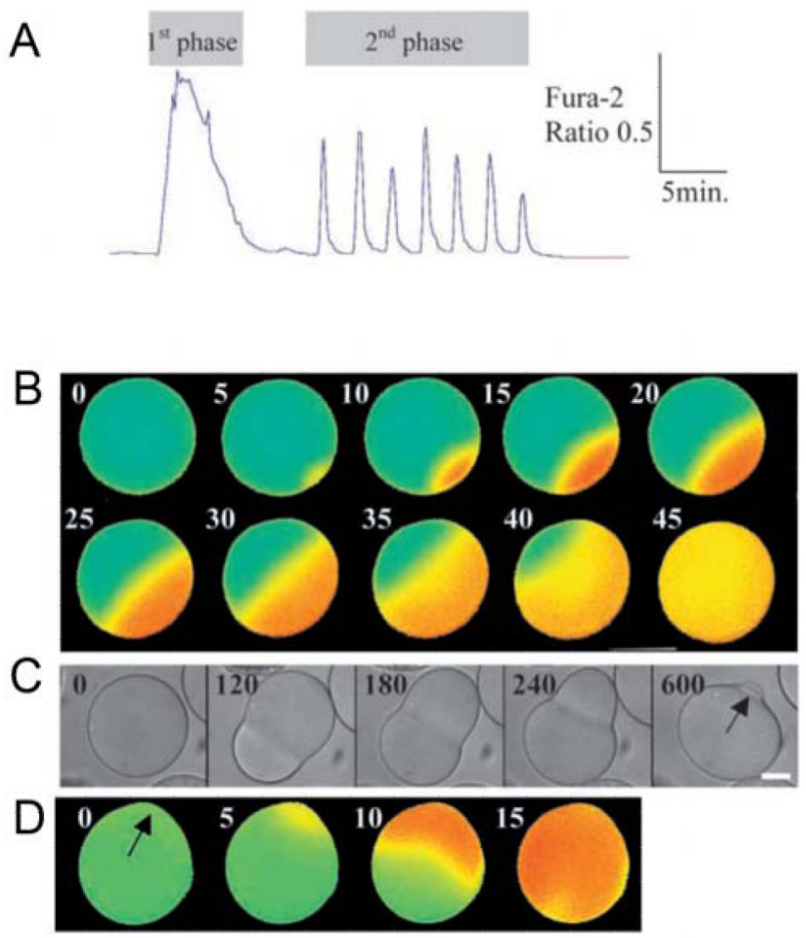

\section{Figure 10.}

Calcium waves in ascidian oocytes at fertilization.

A: the temporal sequence of calcium waves. There is a single large fertilization wave (1st phase), followed by seven equally spaced waves as the oocyte proceeds through the second meiotic division (2nd phase). B: The oocyte is fertilized by the sperm between four and five o'clock and the large fertilization wave sweeps across this $200 \mu \mathrm{m}$ oocyte in around 40s. C: the consequence of the fertilization wave is a very marked cortical contraction that generates a nipple-like cortical protrusion called the contraction pole (arrow). D: the calcium waves of the second phase originate at the contraction pole; a single wave is illustrated. Ascidiella apersa. Reproduced from reference 65. 

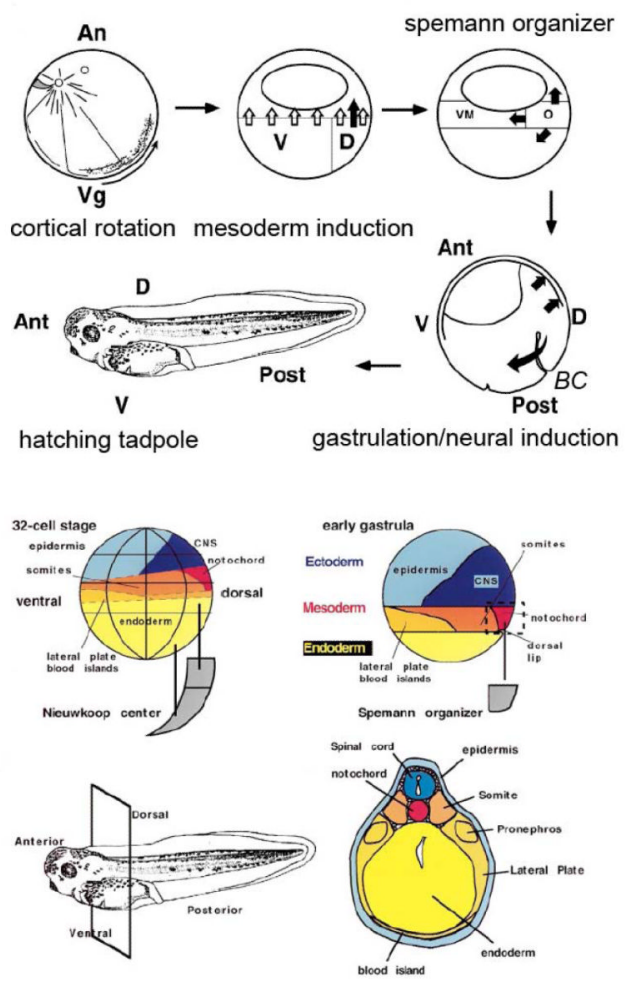

Figure 11.

Pattern formation and cell fate in Xenopus embryos.

ABOVE: cortical rotation involves a displacement of cortical cytoplasm directed along microtubules and specified by the growth of the sperm aster ( $\mathrm{An}=$ animal pole, $\mathrm{Vg}=\mathrm{vegetal}$ pole); mesoderm induction occurs in midblastula after around 12 cell divisions when a blastocoel has formed due to signaling from endoderm to ectoderm ( $V=$ ventral endoderm, $\mathrm{D}=$ dorsal endoderm, open arrows=general induction signal, closed arrow $=$ dorsalizing induction signal from the Nieuwkoop centre); the Spemann organizer (O) emits local signals that induce neural tissues (up), dorsalize mesoderm (left, VM=ventral mesoderm) and anteriorize endoderm (down and left); during gastrulation mesoderm that has come to lie under dorsal extoderm as a consequence of gastrulation movements causes further neural induction (small double arrow), while dorsalization of mesoderm continues through signaling across BC, Brachet's cleft (Ant=anterior, Post=posterior, D=dorsal, V=ventral); the axes of the hatching tadpole are illustrated. BELOW: fate map of early embryo with disposition of tissue illustrated in a cross section of a hatching tadpole. Xenopus laevis, adapted from Richard Harland and John Gerhart (202). 


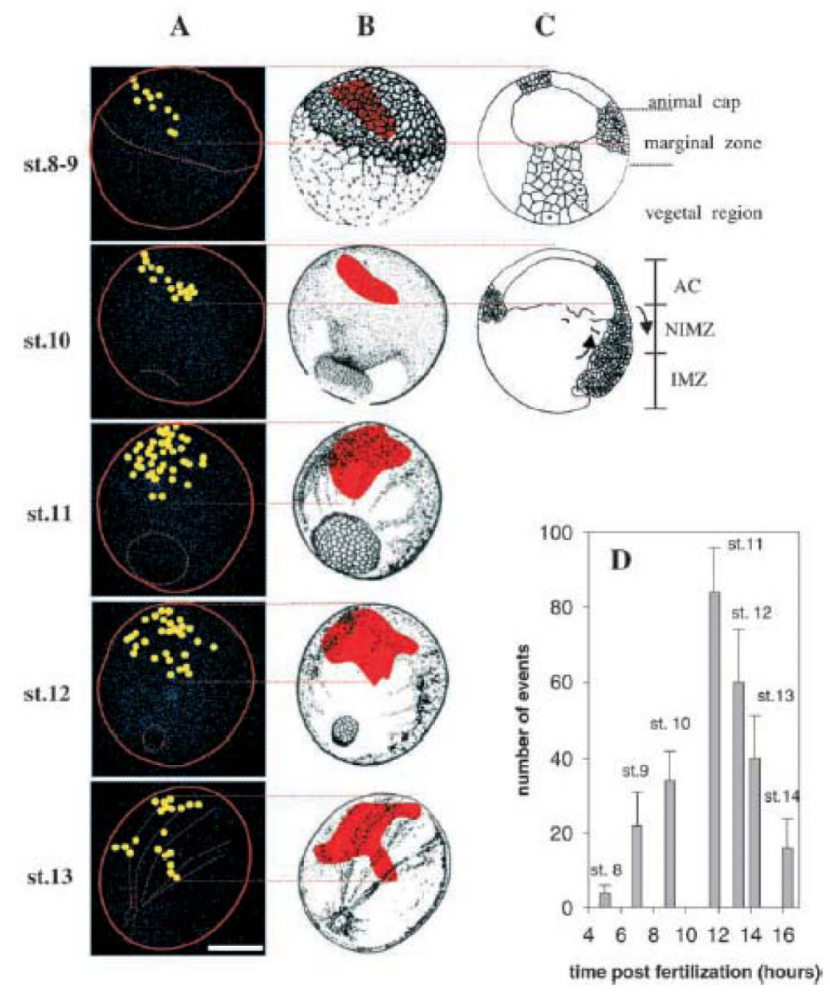

Figure 12.

Localization of calcium pulses during gastrulation in Xenopus laevis.

The point of origin of each calcium pulse is shown in A and line drawings constructed from video images of the same embryo are shown in $\mathrm{B}$, with the area in which calcium pulses appear shown in red. The cross sections in $\mathrm{C}$ are from standard illustrations of Xenopus development. Calcium pulses are confined to dorsal ectoderm throughout gastrulation. Their frequency at different stages of development is shown in $\mathrm{D}$. AC=animal cap, NIMZ=noninvoluting marginal zone, IMZ=involuting marginal zone. Reproduced from reference 305. 


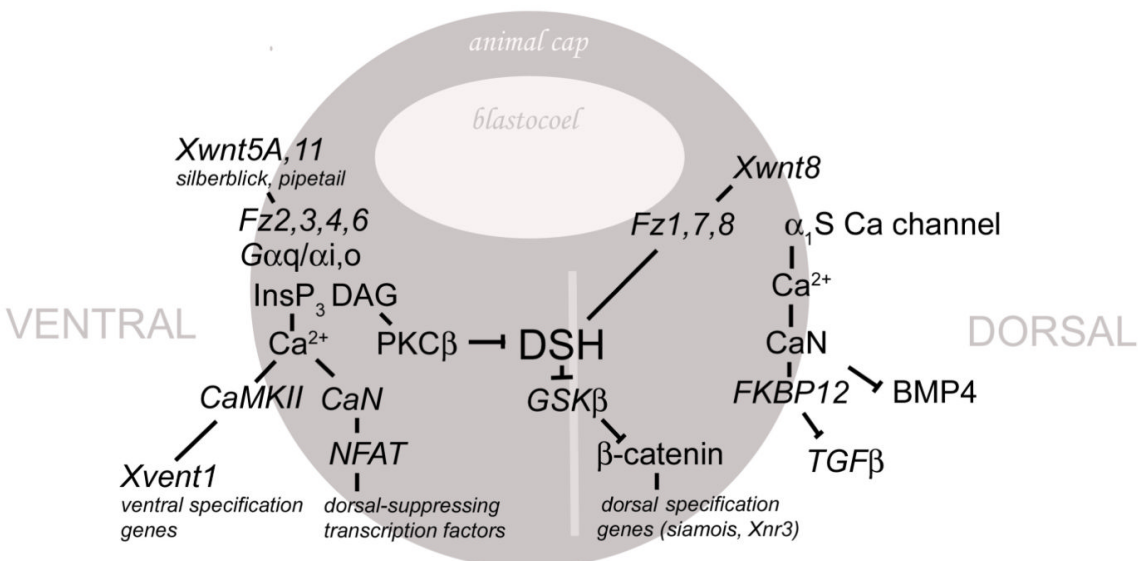

Figure 13.

Calcium signalling pathways during dorso-ventral specification in Xenopus laevis and zebrafish.

Wnt regulated calcium signals promote ventral cell fates via G-protein transduction mechanisms and act through CaM kinaseII and calcineurin to upregulate ventral transcription factors and downregulate dorsal transcription factors; this pathway also downregulates dishevelled signalling via PKC $\beta$, thus antagonizing the canonical dorsalizing Wnt pathway and stabilizing $\beta$-catenin ventrally. A second calcium signalling pathway operates to induce dorsal cell fates. It consists of calcium-channel mediated activation of calcineurin and requires FKBP12; it downregulates the ventralizing BMP4 and TGF $\beta$ pathways. 

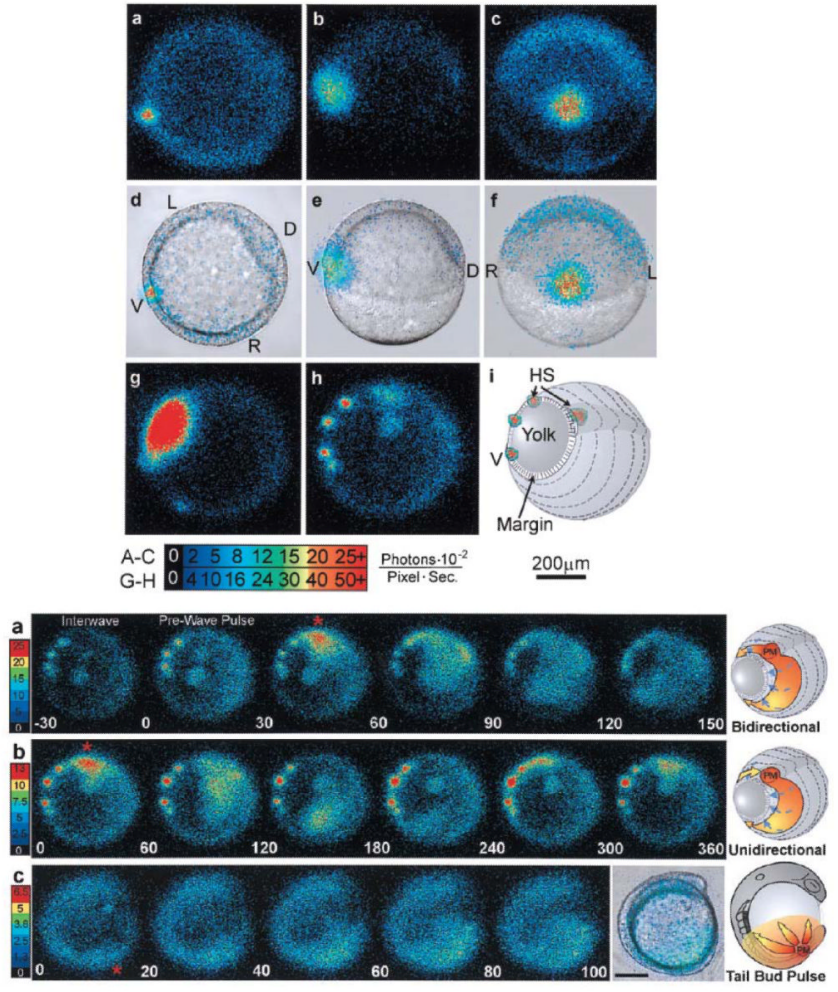

Figure 14.

Localization of calcium pulses during gastrulation in zebrafish.

UPPER PANEL: the three classes of signal during gastrulation between 50 and $75 \%$ epiboly. a-c: three examples of the persistent ventral signal in three difference embryos from three different points of view, shown superimposed on bright field images (d-f). g: yolk flash. h,i: marginal hotspots. $\mathrm{L}=$ left, $\mathrm{R}=$ =right, $\mathrm{D}=$ dorsal, $\mathrm{V}=$ =entral, $\mathrm{HS}=$ hot spots. LOWER PANEL: $a, b$ marginal waves originate from the dorsal midline pacemaker (PM) both unidirectionally and bidirectionally. c tailbud waves. Reproduced from reference 172. 


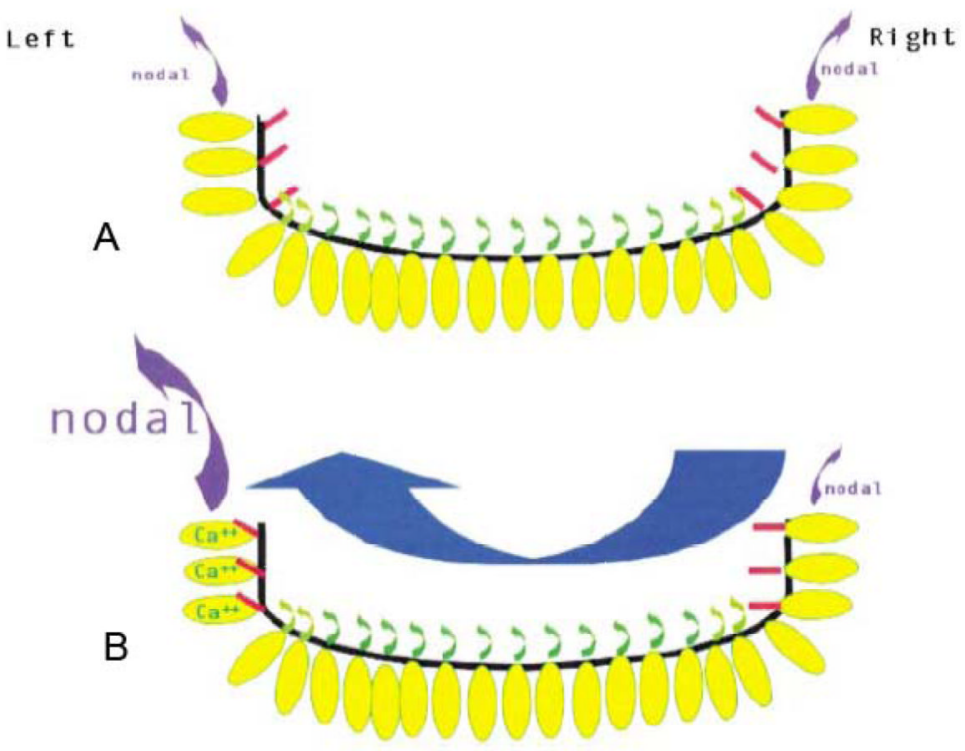

Figure 15.

Calcium and the nodal flow hypothesis of left-right axis determination.

A: Hensen's node is a shallow depression lined with cilia. Central cilia express both dynein and polycystin-2 and are motile (green). Peripheral cilia express only polycystin- 2 and are immotile, with a sensory function (red). At this stage nodal expression shows bilateral symmetry. B: Right to left flow induced by the chiral beating of the motile cilia stimulates a calcium increase in left peripheral sensory cilia, leading to asymmetric expression of nodal. Reproduced from reference 361 . 


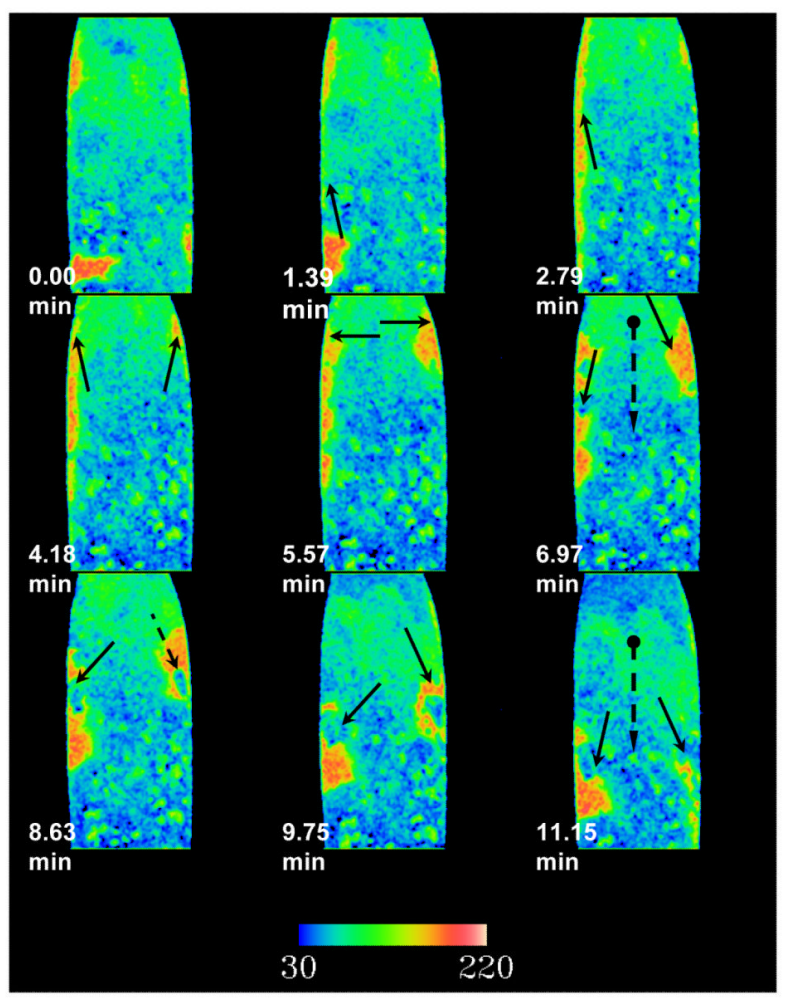

Figure 16.

Calcium waves of unknown function during Drosophila development.

Calcium waves recorded on the ventral aspect on a Bownes stage 9 embryo. Waves move at a velocity of around $2 \mu \mathrm{m} . \mathrm{s}-1$ both anteriorly (0-5min) and posteriorly (5-11 min). The calcium waves are approximately symmetric across the ventral midline and appear to be excluded from specific regions of the embryo (dashed arrow). Waves were visualized using ratiometric confocal fluorescence imaging using calcium green- and rhodamine-dextran. Warm colours represent areas of higher calcium concentration. 


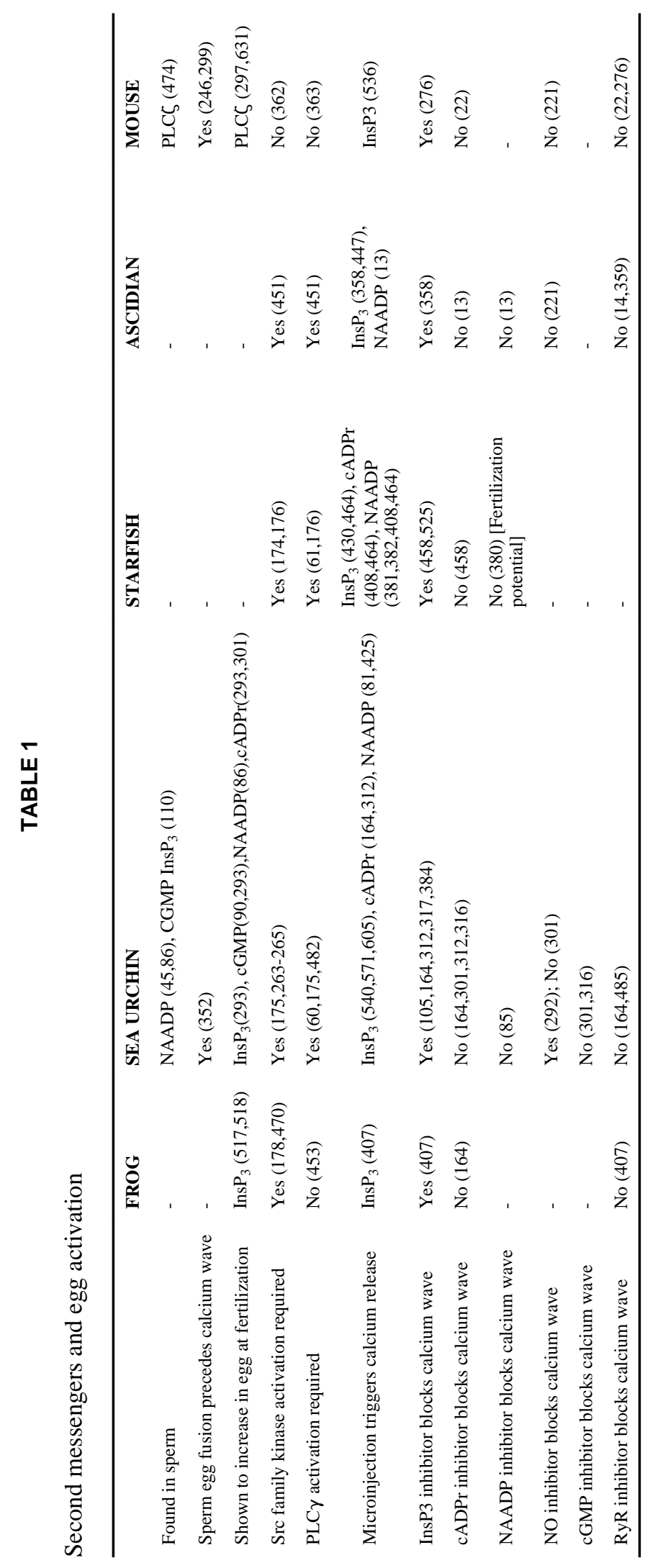

